December 2008

SLAC-PUB-13420

ZU-TH $17 / 08$

$\mathrm{DCPT} / 08 / 138$

$\operatorname{IPPP} / 08 / 69$

Edinburgh 2008/30

FERMILAB-PUB-08-477-T

MCNET/08/14

\title{
Event generation with SHERPA 1.1
}

\author{
T. Gleisberg ${ }^{1}$, S. Höche ${ }^{2}$, F. Krauss ${ }^{3}$, M. Schönherr ${ }^{4}$, S. Schumann ${ }^{5}$, F. Siegert ${ }^{3}$, \\ J. WINTER ${ }^{6}$ \\ ${ }^{1}$ SLAC National Accelerator Laboratory, Stanford University, Stanford, CA 94309, USA \\ ${ }^{2}$ Institut für Theoretische Physik, Universität Zürich, CH-8057 Zürich, Switzerland \\ ${ }^{3}$ Institute for Particle Physics Phenomenology, Durham University, Durham DH1 3LE, UK \\ ${ }^{4}$ Institut für Kern- und Teilchenphysik, TU Dresden, D-01062 Dresden, Germany \\ ${ }^{5}$ School of Physics and Astronomy, The University of Edinburgh, Edinburgh EH9 3JZ, UK \\ (Future address: Institut für Theoretische Physik, Universität Heidelberg, D-69120, Heidelberg, \\ Germany) \\ ${ }^{6}$ Fermi National Accelerator Laboratory, Batavia, IL 60510, USA
}

\begin{abstract}
In this paper the current release of the Monte Carlo event generator Sherpa, version 1.1, is presented. Sherpa is a general-purpose tool for the simulation of particle collisions at high-energy colliders. It contains a very flexible tree-level matrix-element generator for the calculation of hard scattering processes within the Standard Model and various new physics models. The emission of additional QCD partons off the initial and final states is described through a parton-shower model. To consistently combine multi-parton matrix elements with the QCD parton cascades the approach of Catani, Krauss, Kuhn and Webber is employed. A simple model of multiple interactions is used to account for underlying events in hadron-hadron collisions. The fragmentation of partons into primary hadrons is described using a phenomenological cluster-hadronisation model. A comprehensive library for simulating tau-lepton and hadron decays is provided. Where available form-factor models and matrix elements are used, allowing for the inclusion of spin correlations; effects of virtual and real QED corrections are included using the approach of Yennie, Frautschi and Suura.
\end{abstract}




\section{Contents}

1 SHERPA's event generation framework 4

2 Parton distributions and beam spectra 6

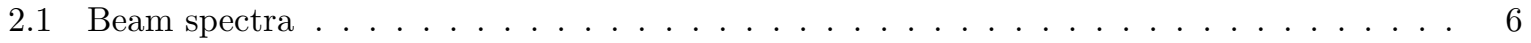

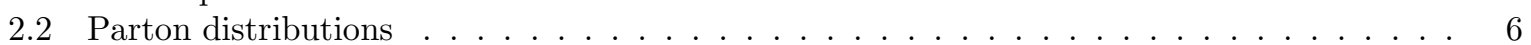

3 Hard matrix elements and phase-space integration $\quad 7$

3.1 Standard Model production processes . . . . . . . . . . . . . . . . . . . . . 10

3.2 Matrix elements for BSM physics . . . . . . . . . . . . . . . . . . . . . . . 11

4 Initial- and final-state radiation $\quad 16$

4.1 The parton cascade APACIC++ . . . . . . . . . . . . . . . . . . . . . . . 16

4.2 Showering off heavy resonances . . . . . . . . . . . . . . . . . . . . . 17

5 Combining LO matrix elements and parton showers $\quad 18$

5.1 The algorithm implemented in SHERPA . . . . . . . . . . . . . . . . . . . . . . . . 19

5.2 Applications . . . . . . . . . . . . . . . . . . . . . . . . 21

6 Multiple parton interactions $r$

7 Parton-to-hadron fragmentation $\quad \mathbf{2 8}$

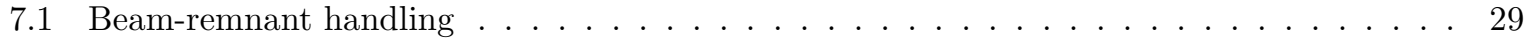

7.2 Cluster-hadronisation model . . . . . . . . . . . . . . . . . . . . . . . . 29

8 Decays of unstable particles $\quad 31$

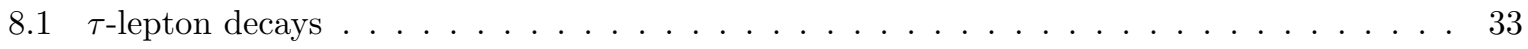

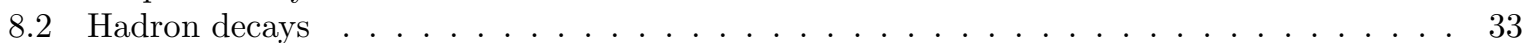

9 Multiple emission of photons $\quad 36$

9.1 The formalism . . . . . . . . . . . . . . . . . . . . 36

9.2 The algorithm . . . . . . . . . . . . . . . . . . . . . 38

$\begin{array}{lr}10 \text { Summary } & 39\end{array}$

\section{Introduction}

The LHC will pose new challenges to both the experimental and the theoretical community. It will operate at the highest centre-of-mass energies ever reached in a collider experiment and provide an enormous luminosity, leading to tremendously large event rates. On the experimental side, the huge phase space in conjunction with the exciting and intricate physics programme of the LHC, ranging from high-precision flavour physics at comparably low scales to the discovery of new particles in the TeV range necessitated the development and refinement of triggers and analysis techniques. In addition, from a more technological point of view, data acquisition, storage and processing therefore required the creation of a world-wide network satisfying the greatly increased computing needs. On the theoretical side, on the other hand, demands for higher precision to correctly model signals of new physics and their backgrounds led to the rethinking of calculation and simulation paradigms and to the construction of a new generation of modern tools. Maybe the most prominent manifestations of these paradigm shifts are the new, full-fledged event generators, which certainly will prove to be indispensable tools for data analysis. Currently, these new simulation programs holding many new features are replacing the wellestablished traditional ones. In many cases, they allow a wider range of applications; for example, the typically more modular frameworks have alleviated the incorporation of new physics models. Often the new tools also offer higher precision in the simulation, because better and more accurate techniques at various stages of the event generation have become available over the past years. Ultimately, this led to a drastic improvement e.g. in the description of Standard Model backgrounds to signals for new physics. Thus it is no surprise that there is a widespread belief that these new tools will have a sizable impact 


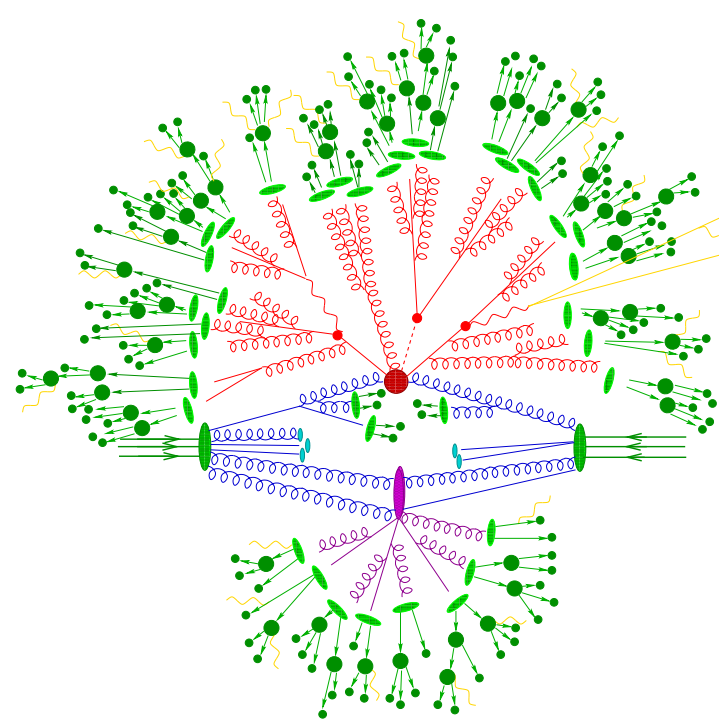

Fig. 1 Pictorial representation of a $t \bar{t} h$ event as produced by an event generator. The hard interaction (big red blob) is followed by the decay of both top quarks and the Higgs boson (small red blobs). Additional hard QCD radiation is produced (red) and a secondary interaction takes place (purple blob) before the final-state partons hadronise (light green blobs) and hadrons decay (dark green blobs). Photon radiation occurs at any stage (yellow).

on the understanding of LHC physics. The construction, maintenance, validation and extension of event generators is therefore one of the principal tasks of particle-physics phenomenology today.

\section{The inner working of event generators}

Fig. 1 pictorially represents a hadron-collider event, where a $t \bar{t} h$ final state is produced and evolves by including effects of QCD bremsstrahlung in the initial and final state, the underlying event, hadronisation and, finally, the decays of unstable hadrons into stable ones. Event generators usually rely on the factorisation of such events into different well-defined phases, corresponding to different kinematic regimes. In the description of each of these phases different approximations are employed. In general the central piece of the event simulation is provided by the hard process (the dark red blob in the figure), which can be calculated in fixed order perturbation theory in the coupling constants owing to the correspondingly high scales. This part of the simulation is handled by computations based on matrix elements, which are either hard-coded or provided by special programs called parton-level or matrix-element (ME) generators. The QCD evolution described by parton showers then connects the hard scale of coloured parton creation with the hadronisation scale where the transition to the colourless hadrons occurs. The parton showers model multiple QCD bremsstrahlung in an approximation to exact perturbation theory, which is accurate to leading logarithmic order. At the hadronisation scale, which is of the order of a few $\Lambda_{Q C D}$, QCD partons are transformed into primary hadrons (light green blobs) by applying purely phenomenological fragmentation models having typically around ten parameters to be fitted to data. The primary hadrons finally are decayed into particles that can be observed in detectors. In most cases effective theories or simple symmetry arguments are invoked to describe these decays. Another important feature associated with the decays is QED bremsstrahlung, which is simulated by techniques that are accurate at leading logarithmic order and, eventually, supplemented with exact first-order results. A particularly difficult scenario arises in hadronic collisions, where remnants of the incoming hadrons may experience secondary hard or semi-hard interactions. This underlying event is pictorially represented by the purple blob in Fig. 1. Such effects are beyond QCD factorisation theorems and therefore no complete first-principles theory is available. Instead, phenomenological models are employed again, with more parameters to be adjusted by using comparisons with data. 


\section{Modern event generators}

The most prominent examples of event generators are the highly successful, well-established programs Pythia [1] and Herwig [2]. They have been constructed over the past decades alongside with experimental discoveries and most of the features visible in past and present experiments can be described by them. However, the need for higher precision to meet the challenges of new energy scales occuring at the LHC, the complexity of final states at those scales, the necessity of maintenance and the wish to easily implement new physics models have demanded those codes to be rewritten in a modern programming language providing a higher level of modularity. Object-oriented frameworks meet the latter requirements, and owing to the community's preference for $\mathrm{C++}$, the new generation of event generators is constructed in this programming language. This led to improved re-implementations in form of the programs PYTHIA 8 [3] and HeRWIG++ [4] - the successors of the Fortran versions mentioned above and to the construction of the SHERPA event generator [5].

In conjunction, in the past decade codes for next-to-leading order calculations have been made available to the public; prominent examples include MCFM [6] and NLOJET++ [7]. Furthermore, methods have been proposed for the consistent matching of next-to-leading order corrections with parton-shower algorithms [8,9]. Corresponding methods are implemented for example in MC@NLO [10], which is based on the Fortran version of HERWIG, in HERWIG ++ [11] and in some more specialised programs [12]. However, the full next-to-leading order calculations underlying these new techniques are very complex and challenging and until today only processes with up to five external legs are under control. On the other hand, many important experimental signatures rely on final states with higher multiplicities. This has triggered substantial activity in perfecting techniques and tools at tree-level accuracy, such that by now several codes are available that can compute corresponding cross sections and generate events in a fully automated way. The most prominent examples include AlPGEN [13], CompHeP/CalcHeP [14], Helac-Phegas [15], MadGraph [16], Whizard [17] and AmegiC++ [18]. Currently only AmegiC++ is part of, and integrated in, a full-fledged event generator, namely the SHERPA framework. In order to translate the multi-particle parton-level events, which are provided by these tools at leading order, into hadron-level events, several algorithms have been developed, all aiming at preserving the logarithmic accuracy of the parton shower and supplementing it with the exact perturbative leading order result for given jet multiplicities. In [19] an algorithm achieving this goal in $e^{+} e^{-}$annihilations into hadrons has been presented and it has been extended to hadronic collisions in [20]. A similar algorithm for the dipole shower has been discussed in [21], whereas a more different one has been published in [22]. All these approaches have been compared in $[23,24]$ and a good agreement has been established.

\section{SHERPA's event generation framework}

SHERPA [5] is an acronym for "Simulation of High Energy Reactions of PArticles". The program is a complete event generation framework that has been constructed from scratch and entirely written in the modern, object oriented programming language $\mathrm{C}++$.

\section{Construction paradigm}

The construction of SHERPA has been pursued in a way largely defined by the following three paradigms:

- Modularity. Different physics aspects are implemented in almost independent modules, relying on a small number of framework and support modules, like, e.g., the event record etc.. Modularity allows, for example, to have more than one matrix-element generator or parton shower in parallel, with the user be in charge of making a choice. The central module, SHERPA, steers the interplay of all other parts and the actual generation procedure.

- Bottom-to-Top. Physics modules are typically developed in their own right, being tested and validated before they are incorporated into the full event generation framework. This in turn results in a quite flexible, minimal structure underlying the organisation of event generation.

- Separation of interface and implementation. In order to facilitate the two requirements above, SHERPA relies on a structure where the (nearly independent) physics modules are accessible only through physics-specific handlers. These handlers assist SHERPA in generating the event at different 
stages, each of which is steered through a specific implementation of Event_Phase_Handler, such as Signal_Process or Jet_Evolution. An example for such an interplay of event phase and physics handler is the Matrix_Element_Handler, enabling the generation of parton-level events either by the built-in hard-coded matrix elements or by the matrix-element generator AMEGIC++. This is relevant for two event stages, the generation of the signal process and owing to the multijet merging procedure the evolution of the jets.

This overall structure fully reflects the paradigm of Monte Carlo event generation by factorising the simulation into well-defined, almost independent phases. Accordingly, each Event_Phase_Handler encapsulates in an abstract way a different aspect of event generation for high-energy particle reactions. This abstraction is then replaced by real physics using handlers, which ensure that the overall event generation framework can be blind to the finer details of the underlying physics and its implementation in form of a physics module.

\section{Physics modules}

In the following the main modules currently distributed with SHERPA will be listed and briefly described. For a more in-depth discussion, the reader will be referred to either the corresponding section in this paper or to the original literature.

\section{- AMEgiC++ [18].}

This is SHERPA's default matrix-element generator based on Feynman diagrams, which are translated to helicity amplitudes using the methods of [25]. It will be described in detail in Sec. 3 . AMEGIC++ has been thoroughly tested for multiparticle production in the Standard Model [26]. Its MSSM implementation has been validated in [27]. AMEGIC++ employs the Monte Carlo phasespace integration library PHASIC. For the evaluation of initial-state (laser backscattering, initialstate radiation) and final-state integrals, the adaptive multi-channel method of [28] is used by default together with a Vegas optimisation [29] of the single channels. In addition, final-state integration accomplished by RAMBO [30] and HAAG [31] is supported.

- $\mathrm{APACIC}++[32,33]$.

APACIC ++ generates initial- and final-state parton showering. The shower evolution is governed by the DGLAP equations and is ordered in parton virtualities. Coherence effects are accounted for by explicit ordering of the opening angles in subsequent branchings. All features needed for a consistent merging with matrix elements $[19,20]$ are included, however, the main part of the merging procedure is implemented in the SHERPA module itself.

- $\mathrm{AmiSIC}++[34]$.

This module simulates multiple parton interactions according to [35]. In SHERPA the treatment of multiple interactions has been extended by allowing the simultaneous evolution of an independent parton shower in each of the subsequent collisions. This shower evolution is handled by APACIC++.

\section{- AHADIC.}

AHADIC is SHERPA's hadronisation package for translating the partons (quarks and gluons) into primordial hadrons. The algorithm is based on the cluster-fragmentation ideas presented in [36], which are also implemented in the HERWIG event generators. It should be noted that AHADIC, essentially based on [37], indeed differs from the original versions, cf. Sec. 7.2.

\section{- Hadrons [38].}

HADRONS is the module for simulating hadron and $\tau$-lepton decays. The resulting decay products respect full spin correlations (if desired). Several matrix elements and form-factor models have been implemented, such as the Kühn-Santamaría model or form-factor parametrisations from Resonance Chiral Theory for the $\tau$-leptons and form factors from heavy quark effective theory or light-cone 
sum rules for hadron decays. For further details, see Sec. 8.

\section{- Photons [39].}

The Photons module holds routines to add QED radiation to hadron and $\tau$-lepton decays based on the YFS algorithm [40]. The structure of PHOTONS is designed such that the formalism can be extended to scattering processes and to a systematic improvement to higher orders in perturbation theory, cf. [39]. The application of РнOTONS therefore fully accounts for corrections that are usually added by the application of Pнотоs [41].

SHERPA itself is the steering module that initialises, controls and evaluates the different phases during the process of event generation. Furthermore, all routines for the combination of parton showers and matrix elements, which are independent of the specific parton shower are found in this module. For details concerning the implementation of these routines, see Sec. 5.

In addition to the main modules of SHERPA, there is a set of tools providing basic routines for event generation, general methods for the evaluation of helicity amplitudes, some generic matrix elements, etc.. Interfaces to commonly used structures like the LHAPDF package [42], or SLHA inputs [43] exist as well as interfaces to most frequently used output formats like HEPEVT and HEPMC.

In the following the different phases of event generation and the respective realisation within the SHERPA program are discussed in detail. Some examples are presented to illustrate the capabilities of the code and highlight its special features.

\section{Parton distributions and beam spectra}

Within SHERPA the incoming beam of particles is resolved in two steps.

- Beamstrahlung, which leads to interacting photons in the hard process or photon generation through laser backscattering is described through appropriate spectra.

- The parton content of photons and hadrons is parametrised by appropriate parton distributions.

\subsection{Beam spectra}

In the context of leptonic incoming beams, e.g. at a future linear collider, photon beams can be prepared through laser backscattering off the (potentially polarised) electrons at very high luminosity [44]. The respective scenario can be simulated in SHERPA through a parametrisation that depends on the energy and polarisation of the incoming lepton beams as well as on the concrete laser specifications [45].

When considering collisions of hadronic beams, e.g. at the Fermilab Tevatron or the CERN LHC, there exists a two-photon component of the cross section, which can reasonably be described in the framework of the equivalent-photon approximation (EPA). The EPA relates the hadronic cross section to the interaction cross section of real photons through a two-photon luminosity function [46]. The corresponding implementation in SHERPA has been described in some detail in [47].

\subsection{Parton distributions}

SHERPA provides a variety of interfaces to standard PDF sets, used to parametrise the parton content of incoming particles in hadron collisions. The most commonly employed LHAPDF package [42] is interfaced such that the full wealth of PDF sets contained in this package is made available throughout the code.

If photons generated through laser backscattering or radiated off hadrons or nuclei are themselves resolved, their quark and gluon content may be parametrised by a photon PDF, see for example [48]. In this context the event generation is essentially equivalent to the procedure for hadronic initial states, although with varying centre-of-mass energy. This can easily be understood, since on the perturbative side of the simulation it only matters that there is a PDF. No information about the incoming beam being a photon is needed. 


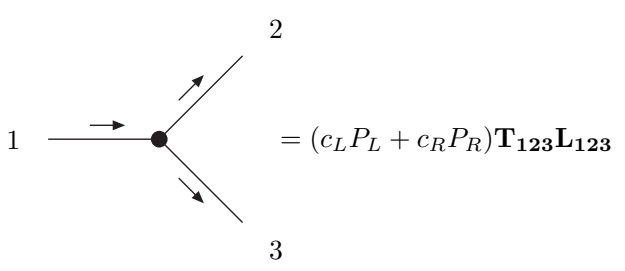

Fig. 2 Generic form of a three-point interaction vertex. The interaction of particles 1,2 and 3 , with 1 incoming while 2 and 3 are outgoing, is defined by the left- $\left(c_{L}\right)$ and righthanded $\left(c_{R}\right)$ coupling, the $S U(3)$ colour operator $\mathbf{T}$, and the Lorentz structure of the interaction $\mathbf{L}$.

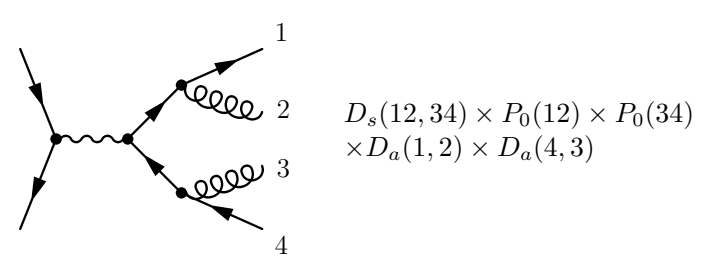

Fig. 3 Translation of a Feynman diagram into a phase-space parametrisation. $D_{s, a}$ denote symmetric or asymmetric decays - the latter ones reproduce the typical feature of collinear emissions of particles notorious for gauge theories with massless spin-one bosons. The propagator terms for massless particles, $P_{0}$, peak at the minimally allowed invariant mass.

\section{Hard matrix elements and phase-space integration}

Matrix elements for the hard scattering process can be provided either by a fast internal library of hard-coded $2 \rightarrow 2$ processes or in an automated way by using the matrix element generator AMEGIC ++ . AMEGIC++ [18], acronym for "A Matrix Element Generator in C++", is a multi-purpose parton-level generator. It is a convenient tool for calculating cross sections of nearly arbitrary scattering processes at tree level based on e.g. the following models:

- Standard Model,

- Extension of the SM by a general set of anomalous triple and quartic gauge couplings [49],

- Extension of the SM by a single complex scalar [50],

- Extension of the SM by a fourth generation,

- Extension of the SM by an axigluon [51],

- Two-Higgs-Doublet Model,

- Minimal Supersymmetric Standard Model,

- ADD model of large extra dimensions [52].

Besides calculating production and decay rates AMEGIC++ is used to generate parton-level events within the event simulation framework of SHERPA. Therefore, these partonic events can easily be supplemented by parton showers and linked to the hadronisation, which in turn yields realistic hadronic final states.

Given a physics model and a certain process AMEGIC++ automatically generates the corresponding set of tree-level Feynman diagrams from the complete set of interaction vertices possessed by the model. ${ }^{1}$ A vertex is thereby defined by the incoming and outgoing particles, the left- and right-handed coupling, the explicit $S U(3)$ colour structure of the interaction, and the related Lorentz structure. For a schematic representation of a generic three-point function, see Fig. 2. Four-point interactions have one more outgoing particle, otherwise are set up completely equivalently.

The available colour operators $\mathbf{T}$ are $1, \delta_{i j}, \delta_{a b}, T_{i j}^{a}, f_{a b c}$ and products of those, e.g. $f_{a b e} f_{c d e}$. Some vertices are decomposed into several colour or Lorentz structures, for example the 4-gluon vertex is represented by three products of colour structures with a respective Lorentz operator. Because of this quite general and explicit treatment of colour new physics models can be implemented in a straightforward manner even though they might imply interactions with colour structures absent in the SM.

The Feynman diagrams constructed from the vertices are translated into helicity amplitudes relying on a formalism similar to the one described in [25] and extended to include also spin-two particles in [53]. During this translation process the chained Lorentz structure of each single diagram is mapped onto

\footnotetext{
${ }^{1}$ Note that unitary gauge is considered for the vertices; moreover AMEGIC++ is currently limited to three- and four-point interactions.
} 

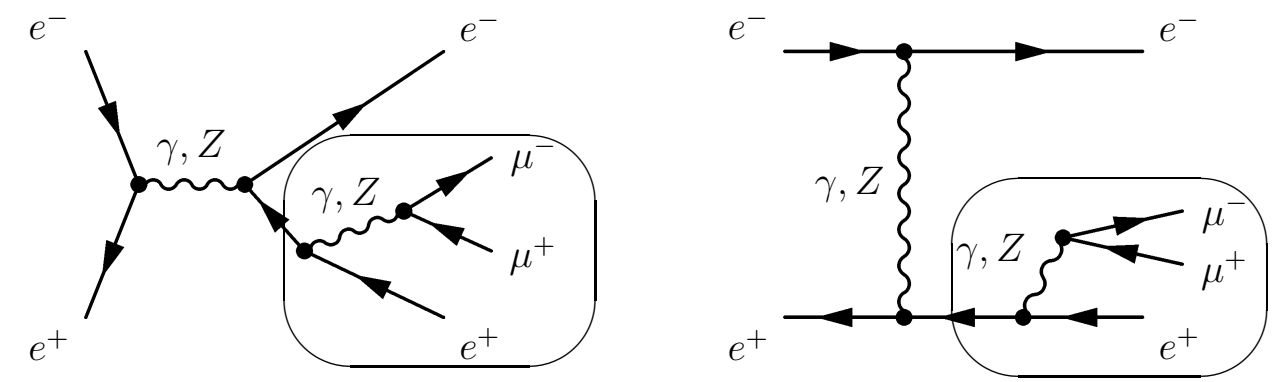

Fig. 4 Factoring out common pieces of amplitudes with identical colour structure. The subgraphs within the boxes are equal, hence, can be factored out such that the two amplitudes can be added.

suitable products and sums of helicity-amplitude building blocks. The diagrams are then grouped into sets of amplitudes with a common colour structure. Based on them, the exact matrix of colour factors between amplitudes is explicitly calculated using $S U(3)$ algebra relations. A number of refinements of the helicity method have been implemented in the code to eventually speed up the matrix-element evaluation and cope with issues that arise when dealing with extensions of the SM. Concerning the latter, cf. Sec. 3.2.1.

For external massive or massless gauge bosons, explicit polarisations are enabled. This allows to calculate polarised cross sections. Furthermore, the numerators of spin-one and spin-two propagators can be replaced by sums over suitably defined polarisations for off-shell particles, thereby disentangling nested Lorentz structures emerging for amplitudes with many internal bosons. As a result, the generic 3- and 4-point Lorentz structures of the considered physics model are the only basic helicity-amplitude building blocks, which are needed by AMEGIC++ to construct arbitrary processes. The complete sets of vertex structures appearing in the SM, the MSSM and the ADD model, plus a comprehensive list of generalised triple and quartic gauge-boson interactions have been implemented. Fortunately, these sets also form the basis for many other extensions of the Standard Model and, hence, such BSM models can be incorporated without the need to provide new helicity-amplitude building blocks.

Employing the helicity formalism allows to accelerate the matrix-element evaluation by making use of symmetries among different Feynman graphs. One example is given by diagrams with equal colour structure that have common factors, as it is illustrated in Fig. 4. Accordingly, identical sub-amplitudes can be factored out and the cut graphs can be added, thereby reducing the number of complex multiplications to be carried out. The optimised helicity amplitudes for the particular process are stored in library files.

The second main task, which needs to be accomplished by AMEGIC++, is the generation of suitable phasespace integrators, which allow an efficient cross-section evaluation and subsequent event generation. The employed algorithm combines process-specific a priori knowledge about the integrand with self-adaptive Monte Carlo integration techniques [28, 29, 54].

The Feynman diagrams for a given process are analysed to create specific phase-space mappings, which generate non-uniform momentum distributions with weights that approximate the corresponding (squared) diagram. The phase space for a single diagram is parametrised using invariant masses of the diagram's propagators and variables related to the angles in particle splittings. As exemplified in Fig. 3 the mapping (and the corresponding weight function) can be assembled out of a few generic building blocks generating corresponding weighted distributions of those parameters. As the matrix elements themselves, all phasespace mappings are stored in library files. For the integration of a scattering process, all contributing channels are combined in a self-adaptive multi-channel integrator, which automatically adjusts to the relative importance of the single phase-space maps to minimise the variance.

The efficiency of the integrator is further improved by applying the self-adaptive VEGAS algorithm on single phase-space maps. VEGAS [29] is very efficient in the numerical adaptation to functions, which are factorisable into a product of one-dimensional functions. Although this is clearly unlikely for a full matrix element, the structure represented by a single phase-space mapping fulfills this condition. VEGAS thus allows an adaptation to more detailed structures generated e.g. by phase-space cuts or the spin of the particles. These structures clearly are beyond the approximations inherent in the mappings. 
Both the helicity amplitudes and the phase-space parametrisations, are semi-automatically ${ }^{2}$ compiled and linked to the code before the actual integration and event generation can start.

In recent versions of SHERPA several improvements to the algorithm outlined above have been implemented. They shall briefly be discussed here.

- Besides calculating the full set of Feynman diagrams for a given final state it is now possible to evaluate only certain resonant graphs from decays of unstable intermediate particles. Accordingly, the process is decomposed into the actual production and the decay(s) of the resonance(s). For a number of intermediate states, labelled $i$, the combined amplitude reads

$$
\mathcal{A}^{(n)}=\mathcal{A}_{\text {prod }}^{\left(n_{\text {prod }}\right)} \otimes \prod_{i \in \text { decays }} \mathcal{P}_{i} \mathcal{A}_{\mathrm{dec}_{i}}^{\left(n_{i}\right)} .
$$

The $\otimes$ symbol represents the colour and spin correlations between the production and decay amplitudes. The factor $\mathcal{P}_{i}$ accounts for the intermediate propagator. Two options are realised: either the resonances are forced on their mass shell, or the full off-shell propagator is considered. Both approaches fully preserve colour and spin correlations, however, only the first method yields strictly gauge-invariant results, owing to the usage of on-shell matrix elements for production and decays. Although gauge invariance cannot be guaranteed when the full off-shell propagator is considered, this ansatz naturally incorporates finite-width effects.

This decay treatment can be applied iteratively, therefore, provides a very elegant way to simulate entire decay chains as they appear for example in supersymmetric theories. Furthermore, the treatment allows for an easy incorporation of $n$-body decays as the decay amplitudes $\mathcal{A}_{\text {dec }}$ are not restricted to $1 \rightarrow 2$ processes.

- Despite the optimisation strategies employed in AMEGIC++ the evaluation of Feynman diagrams for purely strong-interacting processes, in particular $n$-gluon scattering, is still quite cumbersome. For this case, the matrix elements can be evaluated using Cachazo-Svrček-Witten vertex rules [55], which are based on maximal helicity violating (MHV) amplitudes as building blocks. For small and intermediate particle multiplicities, typically up to $2 \rightarrow 5$, these rules give the most compact expressions, cf. [56].

Algorithmically there is no upper limit on particle multiplicities for which matrix elements can be generated. Practically, calculations are however limited by the accessible computer power. Another important criterion is given by comparing AMEGIC++'s performance to alternative approaches for matrix-element generation, e.g. $[13, ?, 57]$. Using the Feynman diagrammatic approach (including the phase-space integration method described above), AMEGIC++ performs reasonably and can compete for scattering processes that typically involve up to a few thousand diagrams. In the framework of the Standard Model this is sufficient for processes with a total number of up to eight or nine particles, or a maximum of six partons in the context of evaluating hadronic cross sections. The latter limit is not only due to the proliferation in the number of Feynman diagrams but also the number of different parton-level processes contributing to a jet cross section, which typically are all desired to be taken into account at once. Accessible particle multiplicities can of course be increased by employing the above mentioned decay treatment, since it restricts the number of amplitudes by accounting for certain resonant graphs only.

The built-in matrix-element generator AMEGIC++ renders SHERPA a very powerful tool to study hard production processes, in particular many-particle final states. The underlying tree-level calculations thereby naturally account for aspects of multi-particle production processes that often are only approximated in alternative approaches. Thus, a clear strength of complete matrix-element calculations is the proper incorporation of off-shell (finite-width) effects and quantum interferences between different diagrams contributing to the same final state. The correct treatment of angular correlations between final-state particles can be achieved as well. All of these aspects are important in approaching a realistic description of both signal and background processes.

\footnotetext{
${ }^{2}$ In detail this means that after a first run generating all process libraries, these libraries have to be compiled and dynamically linked to the main code by executing the automatically generated "makelibs" script.
} 


\begin{tabular}{|l|c|c|c|c|c|}
\hline$\sigma[\mathrm{pb}]$ & \multicolumn{5}{|c|}{ Number of jets } \\
\hline$e^{+} \nu_{e}+$ QCD jets & 0 & 1 & 2 & 3 & 4 \\
\hline AMEGIC++ & $5432(5)$ & $1279(2)$ & $466(2)$ & $185.2(5)$ & $77.3(4)$ \\
Comix & $5434(5)$ & $1274(2)$ & $465(1)$ & $183.0(6)$ & $77.5(3)$ \\
\hline
\end{tabular}

\begin{tabular}{|l|c|c|c|c|c|}
\hline$\sigma[\mathrm{pb}]$ & \multicolumn{5}{|c|}{ Number of jets } \\
\hline$e^{-} e^{+}+$QCD jets & 0 & 1 & 2 & 3 & 4 \\
\hline AMEGIC++ & $723.0(8)$ & $188.2(3)$ & $69.6(2)$ & $27.21(6)$ & $11.1(1)$ \\
Comix & $723.5(4)$ & $187.9(3)$ & $69.7(2)$ & $27.14(7)$ & $11.09(4)$ \\
\hline
\end{tabular}

\begin{tabular}{|l|c|c|c|c|}
\hline$\sigma[\mu \mathrm{b}]$ & \multicolumn{4}{|c|}{ Number of jets } \\
\hline jets & 2 & 3 & 4 & 5 \\
\hline AMEGIC++ & $331.0(4)$ & $22.78(6)$ & $4.98(1)$ & $1.238(4)$ \\
Comix & $331.0(4)$ & $22.72(6)$ & $4.95(2)$ & $1.232(4)$ \\
\hline
\end{tabular}

\begin{tabular}{|l|c|c|c|}
\hline$\sigma[\mathrm{pb}]$ & \multicolumn{3}{|c|}{ Number of jets } \\
\hline$t \bar{t}+$ QCD jets & 0 & 1 & 2 \\
\hline AMEGIC++ & $754.4(3)$ & $747(1)$ & $520(1)$ \\
Comix & $754.8(8)$ & $745(1)$ & $518(1)$ \\
\hline
\end{tabular}

Tab. 1 Comparison of Standard Model production cross sections at the LHC using the MC4LHC parameter setup [61]. In parentheses the statistical error is stated in units of the last digit of the cross section.

\subsection{Standard Model production processes}

All generic interactions stemming from the Standard Model Lagrangian have been implemented, allowing the generation of matrix elements for arbitrary processes involving all three generations of quarks and leptons, all SM gauge bosons and the Higgs boson.

In addition to the generic SM interactions effective interaction vertices between a Higgs boson and massless gauge bosons are available in AMEGIC++. The coupling to gluons is mediated by a top-quark loop and modelled through the effective Lagrangian [58]

$$
\mathcal{L}_{g g H}^{\mathrm{eff}}=g_{g g H} \frac{\alpha_{S}}{2 \pi v} G_{\mu \nu}^{a} G_{a}^{\mu \nu} H
$$

where $G_{\mu \nu}^{a}=\partial_{\mu} A_{\nu}^{a}-\partial_{\nu} A_{\mu}^{a}-g f^{a b c} A_{\mu}^{b} A_{\nu}^{c}$ is the gluon field-strength tensor. The effective coupling $g_{g g H}$ can be calculated either for finite top-quark mass or in the limit $m_{t} \rightarrow \infty$.

For the coupling of a Higgs boson to photons, mediated by top-quark and W-boson loops, the effective Lagrangian reads

$$
\mathcal{L}_{\gamma \gamma H}^{\mathrm{eff}}=\frac{g_{\gamma \gamma H}}{v} F_{\mu \nu} F^{\mu \nu} H
$$

where $F_{\mu \nu}=\partial_{\mu} A_{\nu}^{a}-\partial_{\nu} A_{\mu}^{a}$ is the photon field-strength tensor.

The validation of SHERPA's core - providing the matrix-element calculations - is an important aspect in the development of the SHERPA Monte Carlo generator. AMEGIC++ has successfully been tested against various other programs for a great variety of processes. This includes cross sections and distributions for photon-photon collisions [59], $e^{+} e^{-}$annihilations at different centre-of-mass energies [60,26], and particle production in hadron-hadron collisions [61]. In Tab. 1 exemplary results of a cross-section comparison for some LHC key processes are presented. For this comparison between AMEGIC++ and the independent matrix-element generator COMIX, the calculational setup used in [61] has been employed. The results of both codes agree very well within the statistical uncertainties, indicated by the numbers in parentheses, proving the correctness of the respective calculations.

To illustrate the relevance of accounting for spin correlations we consider the process $\gamma \gamma \rightarrow W^{+} W^{-} \rightarrow$ $l l^{\prime}+\mathbb{E}_{T}$ at the CERN LHC, either in the continuum or mediated by a Standard Model Higgs boson of mass $m_{H}=160 \mathrm{GeV}$. Figure 5 shows the azimuthal separation of all possible lepton combinations emerging from the $W$-decays. Apparently, this observable provides an excellent handle on the irreducible 


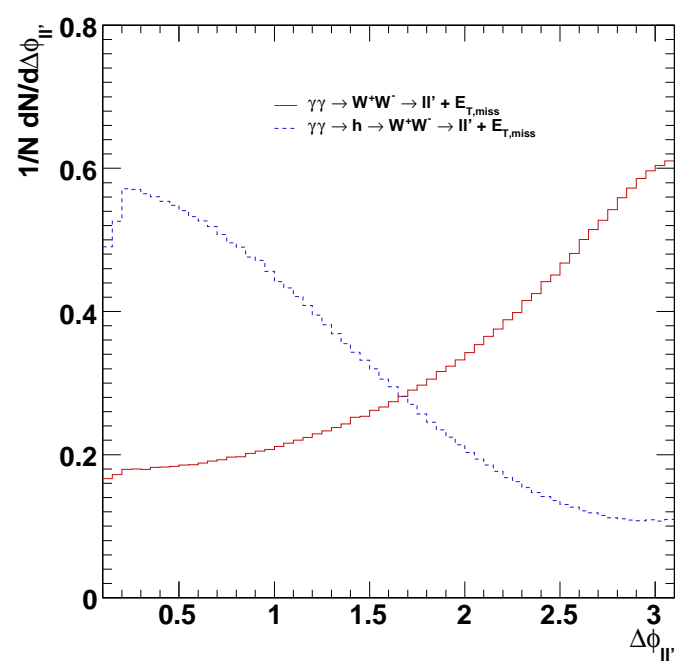

Fig. 5 Lepton azimuthal separation in $\gamma \gamma \rightarrow W^{+} W^{-} \rightarrow l l^{\prime}+\mathbb{E}_{T}$ with and without an intermediate Standard Model Higgs boson of $m_{H}=160 \mathrm{GeV}$.

$W^{+} W^{-}$background process. Note that both spectra have been normalised to unity in order to compare their respective shapes. To parametrise the initial-state photon energy, the spectra described in Sec. 2 have been employed.

\subsection{Matrix elements for physics beyond the Standard Model}

The implementation of new physics models into AMEGIC++ proceeds in two steps. First, the new particles and the parameters of the model have to be declared and corresponding setting routines need to be provided. ${ }^{3}$ Second, the generic interaction vertices of the theory have to be defined. For each new threeand four-point function, this includes the specification of the incoming and outgoing particles and the couplings, as well as the assignment of the $S U(3)$ colour structure of the interaction and its Lorentz structure. Both steps are centralised in the module MODEL such that the parametric quantities of the model can consistently be used in all SHERPA modules.

The only limitation of the AMEGIC++ approach, concerning its extension to new physics scenarios, is the number of Lorentz structures or helicity-amplitude building blocks known to the program. Behind each Lorentz structure there resides a corresponding calculational method for the evaluation of a respective sub-amplitude. Although implementing a new building block is a well defined task, users that wish to do so should contact the authors for advice and help. However, there already exists a large variety of interaction operators for physics beyond the SM. In the following the new physics models available in AMEGIC++ are briefly reviewed and some implementation details will be highlighted.

\subsubsection{The MSSM implementation}

For the Feynman rules of the $R$-parity conserving Minimal Supersymmetric Standard Model, the conventions of [62] are used. The general set of interaction vertices derived there, and as such implemented in AMEGIC++, include not only a full inter-generational mixing of the squark and slepton fields but also permit the inclusion of $\mathrm{CP}$ violating parameters. Furthermore, they include finite masses and Yukawa couplings for all three fermion generations.

Necessary ingredients when dealing with the MSSM are specific Feynman rules for Majorana fermions or fermion number violating interactions. To unambiguously fix the relative signs among Feynman diagrams involving Majorana spinors the algorithm described in [63] is used and the general set of fermion Feynman rules given therein has been implemented. Accordingly, the explicit occurrence of charge-conjugation matrices is avoided, instead a generalised fermion flow is employed that assigns an orientation to complete

\footnotetext{
${ }^{3}$ Essentially this is accomplished by reading numerical values of masses and other parameters from input files.
} 


\begin{tabular}{|c||cc|cc|cc|}
\hline \multicolumn{10}{|c|}{$\sigma\left(\mathbf{W}^{+} \mathbf{W}^{-} \rightarrow \mathbf{X}\right)[\mathrm{fb}]$} \\
\hline Final & \multicolumn{2}{|c|}{ MADGRAPH } & \multicolumn{2}{c|}{ WHIZARD } & \multicolumn{2}{c|}{ SHERPA } \\
state & $\sqrt{s}=0.5 \mathrm{TeV}$ & $\sqrt{s}=2 \mathrm{TeV}$ & $\sqrt{s}=0.5 \mathrm{TeV}$ & $\sqrt{s}=2 \mathrm{TeV}$ & $\sqrt{s}=0.5 \mathrm{TeV}$ & $\sqrt{s}=2 \mathrm{TeV}$ \\
\hline \hline$\tilde{\chi}_{1}^{0} \tilde{\chi}_{1}^{0}$ & $3.8822(2)$ & $1.2741(4)$ & $3.8824(1)$ & $1.27423(8)$ & $3.8821(2)$ & $1.2741(1)$ \\
$\tilde{\chi}_{1}^{0} \tilde{\chi}_{2}^{0}$ & $121.29(1)$ & $24.47(1)$ & $121.2925(7)$ & $24.472(3)$ & $121.296(6)$ & $24.477(1)$ \\
$\tilde{\chi}_{1}^{0} \tilde{\chi}_{3}^{0}$ & $6.8936(7)$ & $12.880(7)$ & $6.8934(2)$ & $12.8790(8)$ & $6.8938(3)$ & $12.8793(6)$ \\
$\tilde{\chi}_{1}^{0} \tilde{\chi}_{4}^{0}$ & $1.4974(1)$ & $9.707(5)$ & $1.4973(6)$ & $9.7064(7)$ & $1.49735(7)$ & $9.7078(4)$ \\
$\tilde{\chi}_{2}^{0} \tilde{\chi}_{2}^{0}$ & $5996.5(4)$ & $1.0415(6) \mathrm{e} 3$ & $5996.57(2)$ & $1.04150(5) \mathrm{e} 3$ & $5996.4(3)$ & $1.04148(5) \mathrm{e} 3$ \\
$\tilde{\chi}_{2}^{0} \tilde{\chi}_{3}^{0}$ & - & $365.6(2)$ & - & $365.615(6)$ & - & $365.63(2)$ \\
$\tilde{\chi}_{2}^{0} \tilde{\chi}_{4}^{0}$ & - & $467.8(2)$ & - & $467.775(8)$ & - & $467.77(2)$ \\
$\tilde{\chi}_{3}^{0} \tilde{\chi}_{3}^{0}$ & - & $82.35(3)$ & - & $82.347(8)$ & - & $82.352(4)$ \\
$\tilde{\chi}_{3}^{0} \tilde{\chi}_{4}^{0}$ & - & $138.20(5)$ & - & $138.18(1)$ & - & $138.205(7)$ \\
$\tilde{\chi}_{4}^{0} \tilde{\chi}_{4}^{0}$ & - & $117.78(4)$ & - & $117.80(1)$ & - & $117.786(6)$ \\
\hline$\tilde{\chi}_{1}^{+} \tilde{\chi}_{1}^{-}$ & $3772(1)$ & $944.3(8)$ & $3771.6(4)$ & $944.2(1)$ & $3771.8(2)$ & $944.32(5)$ \\
$\tilde{\chi}_{2}^{+} \tilde{\chi}_{2}^{-}$ & - & $258.3(2)$ & - & $258.37(4)$ & - & $258.36(1)$ \\
$\tilde{\chi}_{1}^{+} \tilde{\chi}_{2}^{-}$ & - & $131.0(1)$ & - & $130.98(2)$ & - & $130.966(7)$ \\
\hline
\end{tabular}

Tab. 2 Sample cross sections (in fb) calculated using MADGRAPH, WHIZARD and SHERPA for the production of neutral and charged gauginos in $W W$ scattering at fixed centre-of-mass energies of 0.5 and 2.0 $\mathrm{TeV}$. The numbers in brackets reflect the absolute statistical error on the last digit. The considered supersymmetric spectrum corresponds to the SPS1a benchmark scenario [64], generated with SOFTSUSY [65]. Additional details on the calculational setup can be found in [27].

fermion chains. This uniquely determines the external spinors, fermion propagators and interaction vertices involving fermions. Furthermore, negative mass eigenvalues of the physical neutralino fields are taken into account at face value in the propagators and spinor products in the helicity-amplitude expressions. In this way a redefinition of the neutralino fields and couplings can be avoided.

Files that are conform with the SUSY Les Houches Accord (SLHA) [43] are used to input the MSSM weakscale parameters. Such files are provided by external spectrum calculators, see for instance Ref. [65]. The parameters are translated to the conventions of [62] and accordingly fix the couplings of all interaction vertices. Note that so far SHERPA only supports SLHA-v1 and the generalisations presented in [66] have not been implemented yet. This means that further assumptions on the physical model are implicit. The mixing of the squark and slepton fields is restricted to the third generation only and all SUSY breaking parameters and the Yukawa couplings are assumed to be real such that the model realises exact CP symmetry.

To verify the correctness of the implemented Feynman rules and the translation of the SLHA inputs to the conventions of [62], detailed comparisons against the matrix-element generators MADGRAPH [16] and WHIZARD [17], have been carried out [27]. Several hundred cross sections for supersymmetric processes have been compared and mutual agreement between the three independent codes has been found. As an example some of these results are listed in Tab. 2. Besides comparing explicit cross sections, thereby testing all phenomenologically relevant couplings, various unitarity and gauge-invariance checks were performed and supersymmetric Ward- and Slavnov-Taylor identities were tested.

With the complete MSSM Lagrangian being available in a full-fledged matrix element generator like AMEGIC++ detailed aspects of SUSY phenomenology can be studied. Examples include the impact of off-shell kinematics on MSSM signals [67,68], interference effects between various SUSY processes leading to the same final state (irreducible backgrounds) [27], or the impact of additional hard QCD radiation $[69,70]$. Furthermore, non-trivial production processes such as the weak-boson-fusion production of supersymmetric particles can easily be considered [71].

\subsubsection{The ADD model of large extra dimensions}

The Arkani-Hamed-Dimopoulos-Dvali model [52] extends the usual 3+1-dimensional SM by $\delta$ additional compactified spatial dimensions where only gravity can propagate. In this model the usual 4-dimensional 


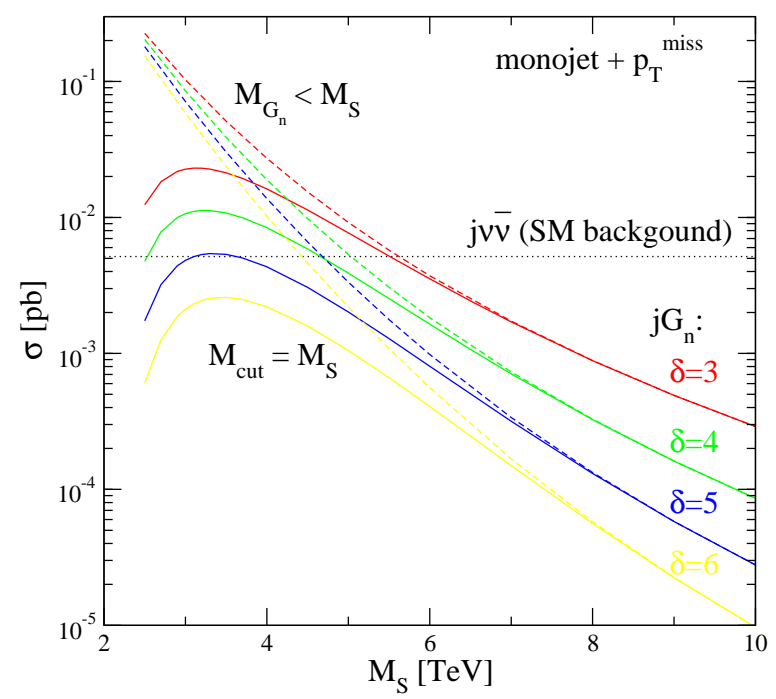

Fig. 6 Dependence of the total cross section on the ADD scale $M_{S}$ for jet-graviton production. The SM background at the LHC is also shown. A phase-space cut of $p_{T}^{\text {miss }}>1 \mathrm{TeV}$ is imposed. The dashed lines are for $M_{G_{n}}<M_{S}$, while the solid lines are for $\sqrt{\hat{s}}<M_{S}$.

Planck scale is related to a fundamental scale $M_{S}$ by

$$
M_{\mathrm{Pl}}^{2}=8 \pi R^{\delta} M_{S}^{\delta+2}
$$

where $R^{\delta}$ is the volume of the compactified extra-dimensional space. A sufficiently large compactification radius $R$ allows $M_{S}$ of the order of $1 \mathrm{TeV}$, providing an explanation of the mass hierarchy between the electroweak and the (4-dimensional) Planck scale. This has observable consequences for TeV-scale colliders. An advantage of this model is that it introduces only two new parameters, the scale $M_{S}$ and the number of extra dimensions $\delta$.

In a 4-dimensional picture the ADD model leads to the coupling of an abundance of Kaluza-Klein graviton states to SM fields. Feynman rules for those interactions can be derived from a linearised gravity Lagragian $[72,73]$.

The AMEGIC++ implementation includes all 3- and 4-point interactions between gravitons and SM fields. It features the generation of matrix elements, which include the exchange of virtual gravitons and the production of real gravitons. To obtain physical cross sections this model additionally requires a summation (usually replaced by an integration) over the Kaluza-Klein states of the graviton. For the two cases this requires different approaches:

- Virtual graviton production:

The integration is performed analytically at the level of the graviton propagator. A number of parametisations appeared in the literature, which include a necessary cut-off for masses above some scale of the order of $M_{S}$. Three widely used conventions have been implemented [72,73,74].

- Real graviton emission:

The sum over accessible Kaluza-Klein states is evaluated together with the phase-space integral using Monte Carlo techniques.

For details of the implementation, cf. [53].

A simple example for the calculation of an ADD cross section with AMEGIC++ is given in Fig. 6, which presents the LHC monojet cross section from ADD graviton production for various numbers of extra dimensions as a function of the scale $M_{S}$ in the notation of [73]. Figure 6 also shows the SM background from $Z$ bosons decaying into a neutrino pair. ${ }^{4}$

All new Lorentz structures implemented for the ADD model are completely generic. This allows to use them in other models that feature couplings to gravitons.

\footnotetext{
${ }^{4}$ Note that the results presented in Fig. 6 can be compared to those of Fig. 5(a) in [75].
} 


\subsubsection{Anomalous interactions of the SM gauge bosons}

Besides the complete set of SM weak-gauge boson interactions AMEGIC++ includes a number of effective Lagrangians describing anomalous triple and quartic gauge interactions. To account for the missing UV completion of the effective theory approach, a simple unitarisation method can be applied.

\section{- $\underline{W W V \text { interactions: }}$}

The general set of operators describing the interaction of two charged vector bosons and a neutral one,

$$
\begin{aligned}
\mathcal{L}_{W W V} / g_{W W V}= & i g_{1}^{V}\left(W_{\mu \nu}^{\dagger} W^{\mu} V^{\nu}-W_{\mu}^{\dagger} V_{\nu} W^{\mu \nu}\right) \\
& +i \kappa_{V} W_{\mu}^{\dagger} V_{\nu} W^{\mu \nu}+\frac{i \lambda_{V}}{m_{W}^{2}} W_{\lambda \mu}^{\dagger} W_{\nu}^{\mu} V^{\nu \lambda} \\
& -g_{4}^{V} W_{\mu}^{\dagger} W_{\nu}\left(\partial^{\mu} V^{\nu}+\partial^{\nu} V^{\mu}\right)+g_{5}^{V} \epsilon^{\mu \nu \rho \sigma}\left(W_{\mu}^{\dagger} \overleftrightarrow{\partial_{\rho}} W_{\nu}\right) V_{\sigma} \\
& +\frac{i \tilde{\kappa}_{V}}{2} \epsilon^{\mu \nu \rho \sigma} W_{\mu}^{\dagger} W_{\nu} V_{\rho \sigma}+\frac{i \tilde{\lambda}_{V}}{2 m_{W}^{2}} \epsilon^{\mu \nu \rho \sigma} W_{\mu \lambda}^{\dagger} W_{\nu}^{\lambda} V_{\rho \sigma},
\end{aligned}
$$

cf. [76], is implemented. Here $V^{\mu}$ denotes either the photon or the $Z$ field, $W^{\mu}$ is the $W^{-}$field, $W_{\mu \nu}=\partial_{\mu} W_{\nu}-\partial_{\nu} W_{\mu}, V_{\mu \nu}=\partial_{\mu} V_{\nu}-\partial_{\nu} V_{\mu}, \tilde{V}_{\mu \nu}=\frac{1}{2} \epsilon_{\mu \nu \rho \sigma} V^{\rho \sigma}$ and $\left(A \overleftrightarrow{\partial_{\mu}} B\right)=A\left(\partial_{\mu} B\right)-\left(\partial_{\mu} A\right) B$ The overall coupling constants are given by

$$
g_{W W \gamma}=-e \quad \text { and } \quad g_{W W Z}=-e \cot \theta_{W} .
$$

The SM values of the individual couplings are $g_{1}^{Z / \gamma}=\kappa_{Z / \gamma}=1$ while all others vanish.

- Quadruple interactions:

The following $S U(2)$ custodial symmetry conserving quartic interactions are available:

$$
\begin{aligned}
& \mathcal{L}_{4}=\alpha_{4} e^{4}\left(\frac{1}{2} W_{\mu}^{\dagger} W^{\dagger \mu} W_{\nu} W^{\nu}+\frac{1}{2}\left(W_{\mu}^{\dagger} W^{\mu}\right)^{2}+\frac{1}{c_{W}^{2}} W_{\mu}^{\dagger} Z^{\mu} W_{\nu} Z^{\nu}+\frac{1}{4 c_{W}^{4}}\left(Z^{\mu} Z^{\mu}\right)^{2}\right), \\
& \mathcal{L}_{5}=\alpha_{5}\left(\left(W_{\mu}^{\dagger} W^{\mu}\right)^{2}+\frac{1}{c_{W}^{2}} W_{\mu}^{\dagger} Z^{\mu} W_{\nu} Z^{\nu}+\frac{1}{4 c_{W}^{4}}\left(Z^{\mu} Z^{\mu}\right)^{2}\right),
\end{aligned}
$$

cf. [77]. In the SM limit the parameters $\alpha_{4}$ and $\alpha_{5}$ are identical zero.

\section{- $\gamma$ - $Z$ interactions:}

The general anomalous coupling of two on-shell neutral gauge bosons $\left(V_{1}\right.$ and $\left.V_{2}\right)$ to an off-shell boson $\left(V_{3}\right)$ has been given in [76]. The corresponding Feynman rule is displayed in Fig. 7. The vertex functions $\Gamma_{V_{1} V_{2} V_{3}^{*}}$ for $V_{2}=Z$ and $V_{2}=\gamma$ are given in Eqs. (9) and (10), respectively. In the $\mathrm{SM}$ all coupling parameters, i.e. $f_{4}^{V}, f_{5}^{V}, h_{i}^{V}$, equal zero.

$$
\begin{gathered}
\Gamma_{Z Z V^{*}}^{\alpha \beta \mu}\left(q_{1}, q_{2}, P\right)=\frac{i\left(P^{2}-m_{V}^{2}\right)}{m_{Z}^{2}}\left[f_{4}^{V}\left(P^{\alpha} g^{\mu \beta}+P^{\beta} g^{\mu \alpha}\right)+f_{5}^{V} \epsilon^{\mu \alpha \beta \rho}\left(q_{1}-q_{2}\right)_{\rho}\right] \\
\Gamma_{Z \gamma V^{*}}^{\alpha \beta \mu}\left(q_{1}, q_{2}, P\right)=\frac{i\left(P^{2}-m_{V}^{2}\right)}{m_{Z}^{2}}\left\{h_{1}^{V}\left(q_{2}^{\mu} g^{\alpha \beta}-q_{2}^{\alpha} g^{\mu \beta}\right)\right. \\
\left.\quad+\frac{h_{2}^{V}}{m_{Z}^{2}} P^{\alpha}\left[\left(P q_{2}\right) g^{\mu \beta}-q_{2}^{\mu} P^{\beta}\right]+h_{3}^{V} \epsilon^{\mu \alpha \beta \rho} q_{2 \rho}+\frac{h_{4}^{V}}{m_{Z}^{2}} P^{\alpha} \epsilon^{\mu \beta \rho \sigma} P_{\rho} q_{2 \sigma}\right\}
\end{gathered}
$$

It should be noted that the most general anomalous coupling between three off-shell neutral gauge 


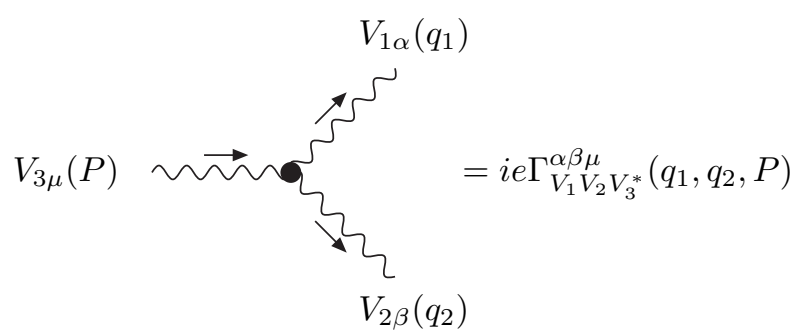

Fig. 7 Feynman rule for the anomalous triple neutral gauge boson vertex.

bosons allows more coupling terms [78], which are, however, not implemented in the current version. To account for this a symmetrised version of the above vertex is used outside the on-shell limit of two of the vector bosons. For example, the interaction of three off-shell $Z$ bosons is modelled through $\Gamma_{Z^{*} Z^{*} Z^{*}}=\Gamma_{Z_{1} Z_{2} Z_{3}^{*}}+\Gamma_{Z_{2} Z_{3} Z_{1}^{*}}+\Gamma_{Z_{3} Z_{1} Z_{2}^{*}}$.

\section{- Unitarisation:}

Owing to the effective nature of the anomalous couplings unitarity might be violated for coupling parameters other than the SM values. For very large momentum transfers, such as those probed at the LHC, this will lead to unphysical results. As discussed in [79] this can be avoided by introducing energy dependent form factors for the deviation of coupling parameters from their Standard Model values:

$$
a(\hat{s})=\frac{a_{o}}{\left(1+\hat{s} / \Lambda^{2}\right)^{n}}
$$

where $\hat{s}$ is the partonic centre-of-mass energy, $\Lambda$ represents the new physics scale, and the exponent $n$ is typically chosen to be $n=2$.

\subsubsection{Further BSM models}

Besides the major model implementations described above AMEGIC++ offers various other physics scenarios that shall briefly be listed here:

- Two-Higgs-Doublet model:

As byproduct of the MSSM implementation the THDM of type II can be studied. In contrast to the MSSM, the relevant input parameters of the extended Higgs sector (masses of the Higgs bosons $h^{0}, H^{0}, A^{0}$, and $H^{ \pm}$, the mixing angle between the two doublets, $\alpha$, and the ratio of their vevs, $\tan \beta$ ) have to be specified explicitly.

\section{- Phantom-Higgs model:}

This model has been discussed in [50]. It emerges by adding a complex scalar gauge singlet to the SM, which interacts with the SM particle through a mixed quartic coupling with the original Higgs doublet of the SM. After symmetry breaking, a massless pseudoscalar and a massive scalar augment the original SM particle spectrum. The latter will undergo a mixing with the original Higgs boson, therefore dilute its couplings to the other SM particles through a mixing parameter. On the other hand, the massless pseudoscalar does not interact with the SM world, apart from the Higgs boson, leading to a sizable invisible partial width of both scalars. The relevant parameters in this model are the masses of the two scalars, their mixing angle, $\tan \theta$, and the ratio of vevs, $\tan \beta$.

- 4th generation:

In this model a fourth generation family has been included, i.e. a lepton $\ell_{4}$, a neutrino $\nu_{4}$, an up-type and a down-type quark $u_{4}$ and $d_{4}$, respectively. They are parametrised by their masses, 
which also serve as input parameters. In addition, both may decay to the Standard Model particles through the charged current weak interaction where the mixing to the other generations is parametrised by one mixing angle each. In the lepton case, this implies that all $\ell_{4}$ and $\nu_{4}$ must decay into a $\tau$-neutrino or a $\tau$-lepton, respectively, while the pattern in the quark case is a bit more involved. There the mixing matrix emerges by multiplying the original $3 \times 3$ CKM matrix with a rotation matrix in the 34 -sector, cf. [80].

\section{- Axigluons:}

Here, an axigluon, i.e. a massive axial vector with the colour quantum numbers and interactions of the gluon is added to the Standard Model, cf. [51]. The only input parameter is the mass of this particle. The implications of such a particle have been discussed, e.g., in [81].

There exist further (though private) implementations of alternative physics scenarios that are not publicly available yet but can be obtained from the respective authors on request, see e.g. Refs. [82, 83]. Note that to further alleviate the implementation of new physics ideas and to allow for an easy distribution an interface to the FEYNRULES package [84] is currently being developed.

\section{Initial- and final-state radiation}

QCD parton evolution and the occurrence of jets can be understood theoretically when the structure of perturbative amplitudes is examined in the kinematical regime of intrajet evolution, i.e. where two or more partons get close to each other in phase space. Whenever this happens, any QCD matrix element squared factorises into a matrix element squared containing the combined "mother" parton and a universal function describing the splitting into the "daughters". In this limit, the theory becomes semi-classical and can be understood in a Markovian approach, where a single initiating parton develops a cascade of independent branchings. This is the basic concept of any shower Monte Carlo. Potential differences then arise in the factorisation scheme only. The default shower generator within SHERPA is APACIC++ [32,33]. It is essentially based on virtuality ordered DGLAP parton evolution with superimposed angluar ordering constraints.

\subsection{The parton cascade APACIC ++}

Final-state showering in APACIC++ proceeds along the lines of [85]. $Q^{2}$ evolution of QCD partons is simulated by $1 \rightarrow 2$ splittings, which occur with differential probability

$$
\frac{\mathrm{d} \mathcal{P}_{\text {branch }, a}^{(F)}}{\mathrm{d} t}=\frac{\mathrm{d}}{\mathrm{d} t} \exp \left\{-\int_{t}^{t^{\prime}} \frac{\mathrm{d} \bar{t}}{\bar{t}} \int_{\tilde{z}_{\min }(\bar{t})}^{\tilde{z}_{\max }(\bar{t})} \mathrm{d} \tilde{z} \frac{\alpha_{s}\left(\mathrm{k}_{\perp}(\tilde{z}, \bar{t})\right)}{2 \pi} \sum_{b=q, g} P_{a b}(\tilde{z})\right\}
$$

Here $a$ denotes the flavour of the splitting parton and $P_{a b}(z)$ are the unregularised Altarelli-Parisi kernels in four dimensions for the splitting $a \rightarrow b c$. The transverse momentum $\mathrm{k}_{\perp}$ is given with respect to the axis defined by the direction of the decayer. The condition $\mathrm{k}_{\perp}>\mathrm{k}_{\perp \text {, min }}$, with $\mathrm{k}_{\perp \text {, min }}$ some cutoff value for $\mathrm{k}_{\perp}$, yields the boundaries of the $\tilde{z}$-integral, $\tilde{z}_{\min }$ and $\tilde{z}_{\max }$, cf. [33]. Evolution and splitting variable are defined by

$$
t=p_{a}^{2}-m_{a}^{2}, \quad \text { and } \quad \tilde{z}=\frac{E_{b}}{E_{a}},
$$

respectively, $m_{a}$ being the on-shell mass of parton $a$. The splitting variable $\tilde{z}$ is related to the light-cone momentum fraction $z=p_{b}^{+} / p_{a}^{+}$(with the "+" direction defined by $p_{a}$ ) through

$$
2 \tilde{z} \kappa E_{a}^{2}+z\left(t_{a}-\kappa^{2} E_{a}^{2}\right)=t_{a}+t_{b}-t_{c} \quad \quad \text { where } \quad \kappa=1+\sqrt{1-\frac{t_{a}}{E_{a}^{2}} .}
$$

Although defined in an apparently non-covariant way, the splitting variable actually is Lorentz invariant [85]. Colour coherence during evolution is taken into account by an explicit angular veto, which 
means that a branching is rejected if the opening angle of the emission is larger than the one of the previous branching.

Initial-state showering proceeds in the backward-evolution picture along the lines of [86]. The differential branching probability reads

$$
\frac{\mathrm{d} \mathcal{P}_{\text {branch }, a}^{(I)}}{\mathrm{d} t}=\frac{\mathrm{d}}{\mathrm{d} t} \exp \left\{-\int_{t}^{t^{\prime}} \frac{\mathrm{d} \bar{t}}{\bar{t}} \int_{x}^{\bar{z}_{\max }(\bar{t})} \frac{\mathrm{d} \bar{z}}{\bar{z}} \frac{\alpha_{s}\left(\mathrm{k}_{\perp}(\bar{z}, \bar{t})\right)}{2 \pi} \sum_{b=q, g} P_{b a}(\bar{z}) \frac{f_{b}(x / \bar{z}, \bar{t})}{f_{a}(x, \bar{t})}\right\}
$$

where the ratio $f_{b}(x / \bar{z}, \bar{t}) / f_{a}(x, \bar{t})$ accounts for the change of parton distributions in each shower step. The splitting variable $\bar{z}$ can be reinterpreted as

$$
\bar{z}=\frac{\hat{s}^{\prime}}{\hat{s}}
$$

$\hat{s}$ and $\hat{s}^{\prime}$ being the partonic center of mass energies before and after the branching, respectively. This immediately yields the relation $x^{\prime}=x / \bar{z}$, thus is partially defining the kinematics after the branching.

An important issue for DGLAP shower algorithms is the convention to implement kinematic constraints once a splitting generates recoil owing to the branching parton going off mass-shell. The recoil strategy seems ambiguous because the branching equations are independent of it. In fact, for the derivation of the DGLAP equation, it may be assumed that there is a spectator parton aligned along the same axis as the splitter, but with opposite direction. This leads to the following approach for APACIC++:

- In final-state branchings the parton, which originates from the same splitting as the branching parton, takes the recoil. If this parton has been already decayed, the decay products are boosted accordingly. This amounts to redefining the splitting variable of the respective branching by

$$
\tilde{z} \rightarrow \tilde{z}^{\prime}=\left(\tilde{z}-\frac{t_{a}+t_{b}-t_{c}}{2 t_{a}}\right) \sqrt{\frac{\left(t_{a}-t_{b}^{\prime}-t_{c}^{\prime}\right)^{2}-4 t_{b}^{\prime} t_{c}^{\prime}}{\left(t_{a}-t_{b}-t_{c}\right)^{2}-4 t_{b} t_{c}}}+\frac{t_{a}+t_{b}^{\prime}-t_{c}^{\prime}}{2 t_{a}}
$$

where $t$ and $t^{\prime}$ denote original and reassigned virtualities, respectively.

- In initial-state branchings all remaining partons take the recoil. For any splitting $b \rightarrow a$, the process is redefined with parton $b$ rather than $a$ aligned along the beam axis and $\hat{s} \rightarrow \hat{s} / \bar{z}$.

The Altarelli-Parisi splitting functions, $P_{a b}(z)$, are taken in the quasi-collinear limit and include mass effects of the emitting parton, cf. [87],

$$
\begin{aligned}
& P_{q q}(z)=C_{F}\left[\frac{1+z^{2}}{1-z}-2 \mu_{q g}^{2}\right] \\
& P_{g q}(z)=T_{R}\left[z^{2}+(1-z)^{2}-\mu_{q \bar{q}}^{2}\right], \\
& P_{g g}(z)=C_{A}\left[\frac{z}{1-z}+\frac{1-z}{z}+z(1-z)\right] .
\end{aligned}
$$

Quark-mass dependencies are given in terms of the dimensionless variable

$$
\mu_{i j}^{2}=\frac{m_{i}^{2}+m_{j}^{2}}{\left(p_{i}+p_{j}\right)^{2}-m_{i j}^{2}} .
$$

APACIC++ has been thoroughly tested and validated [33]. Modifications to the shower algorithm, which were necessary for a proper implementation of the CKKW merging procedure, have been implemented in full generality, see Sec. 5.

\subsection{Showering off heavy resonances}

$\mathrm{APACIC}++$ is also equipped with the possibility to generate radiation off intermediate heavy resonances, once these are described by separable production and decay processes, cf. Sec. 3. An example is top-quark pair production, which plays a significant role, both as a signal for a better measurement of Standard Model parameters and as a background to new physics searches. 
The QCD radiation pattern in heavy-flavour decays has been thoroughly investigated in Refs. [88]. Within APACIC++ a rather simple strategy is employed. QCD radiation off the decaying heavy particle is described by the standard parton shower with massive splitting functions, except for two modifications:

1. In ordinary final-state parton showering, the mother particle goes off-shell, while the daughters retain their respective on-shell masses. In showering off decaying heavy particles, on the contrary, the mother particle retains its on-shell mass, while the daughter of the same flavour goes off-shell with decreased virtuality.

2. The maximally allowed phase-space volume in a branching process of a decaying particle is reduced by the factor

$$
w_{\mathrm{PS}}=\frac{\mathrm{d} \Phi^{(2)}\left(t^{\prime}, t_{b}, t_{c}\right)}{\mathrm{d} \Phi^{(2)}\left(t, t_{b}, t_{c}\right)}=\sqrt{\frac{t^{3}}{t^{3}} \frac{\lambda\left(t^{\prime}, t_{b}, t_{c}\right)}{\lambda\left(t, t_{b}, t_{c}\right)}}
$$

where $\lambda(a, b, c)=(a-b-c)^{2}-4 b c, t$ and $t^{\prime}$ are the virtualities of the decaying particle before and after the emission, respectively, and $t_{b}$ and $t_{c}$ are the virtualities of the decay products. This correction weight corresponds to a decrease in phase-space volume $\mathrm{d} \Phi^{(2)}$ owing to a decrease in three-momentum $\left|\mathrm{p}_{a / b}^{\mathrm{cm}}\right|$ in the centre-of-mass frame of the decayed parton.

To correctly describe the decay process of the heavy flavour, it is vital to respect spin correlations between production and decay amplitudes. Within SHERPA, this is done by firstly computing the full matrix element for production and decay of the heavy flavour and subsequently adding in the parton evolution of the intermediate state. In this respect, it has to be defined, how the kinematics of the decay products is to be reconstructed, once a parton emission has occurred in the shower. The following strategy is used:

1. If the decaying heavy particle keeps its mass, i.e. if the radiation occurs in the production part of the process, the decay products are simply boosted into the new centre-of-mass frame of the decayer.

2. If the decaying heavy particle does not keep its mass, i.e. if the radiation occurs in the decay part of the process, the decay products are reconstructed such that in the centre-of-mass frame of the daughter the momenta point into the same direction as they did in the centre-of-mass frame of the mother before.

This procedure largely retains the correlations between the final-state particles initially described by the matrix element.

\section{Combining LO matrix elements and parton showers}

One of the most important challenges for simulating events at modern high-energy collider experiments is the accurate theoretical description of multijet final states. They constitute the testbed for many new-physics searches, whose success will largely depend on our understanding of the Standard Model multijet production mechanisms. The best theoretical tools available are therefore needed to approach this problem as accurately as possible and obtain reliable estimates of the Standard Model backgrounds to experimental analyses. Underlying hard processes should be accounted for by full matrix-element calculations and the subsequent evolution and conversion of hard partons into hadronic jets should be modelled by QCD parton cascades and phenomenological hadronisation models. However, there are several scales involved that determine the thorough development of an event which makes it difficult to unambiguously disentangle the components belonging to the hard process and the hard-parton evolution. Given an $n$ jet event of well separated partons, its jet structure is retained when emitting a further collinear or soft parton only. An additional hard, large-angle emission, however, gives rise to an extra jet changing the $n$ to an $n+1$ jet final state. Accordingly a merging scheme for matrix element calculations and parton showers has to be defined, that determines on an event-by-event basis which possibility has to be followed. The primary goal is to avoid double counting by preventing events to appear twice, i.e. once for each possibility, as well as dead regions by generating each configuration only once and using the appropriate path. 
Basically two different solutions for this problem have been proposed and implemented in the past few years, the MLM scheme [22], relying on a geometric analysis of the unconstrained radiation pattern in terms of cone jets, and the CKKW scheme or CKKW merging algorithm [19, 20], employing an analytical reweighting of the matrix elements supplemented by a constrained parton-shower evolution. A reformulation of CKKW to a merging procedure in conjunction with a dipole shower (CKKW-L) has been presented in [21]. Common to all schemes is that sequences of tree-level multileg matrix elements with increasing final-state multiplicity are merged with parton showers to yield a fully inclusive sample with no double counting and correct at leading-logarithmic accuracy. On the theoretical side there have been various studies to compare the different approaches, see e.g. Refs. [89,23,24], and the first dedicated experimental analyses on comparing these improved Monte Carlo predictions with actual data have been presented $[90,91,92]$. However, there is more work needed, on the one hand to understand the systematic uncertainties of the various schemes, and, on the other hand further experimental inputs are needed to validate the available tools. This is crucial in prospect of the LHC providing first measurements soon. The SHERPA generator provides a general and largely process-independent implementation of the CKKW algorithm [93], which has extensively been validated for $e^{+} e^{-}$collisions into hadrons [93, 33], the production of single vector bosons at the Fermilab Tevatron [94] and the CERN LHC [95] and for $W^{+} W^{-}$ production at hadron colliders, see [96]. The combined treatment of matrix elements and parton showers is supplemented by a multiple-interactions description, which respects the jet-production scales of the primary process. In the following, these key features of SHERPA shall be discussed in more detail. The actual merging algorithm employed will be presented in Sec. 5.1. Aspects of its application will be discussed in Sec. 5.2.

\subsection{The algorithm implemented in SHERPA}

The idea underlying CKKW is to divide the phase space of partonic emissions according to a $k_{T}$ measure [97] into a regime of jet production, described by appropriate matrix elements, and a regime of jet evolution, described by parton showering. The separation is defined by the merging scale, denoted by $Q_{\text {cut }}$. The matrix elements are reweighted by $\alpha_{s}$ coupling factors and terms that arise from analytic Sudakov form factors. The acceptance or rejection of jet configurations is realised according to this reweighting. Then, each hard parton of the reweighted matrix-element final state undergoes vetoed parton showering, i.e. starting from the scale where this parton appeared first, any new emission that would give rise to an extra jet is vetoed. In this way, the dependence on the, in principle, arbitrary separation scale $Q_{\text {cut }}$ regularising the matrix elements is mostly eliminated and the accuracy of the parton shower is preserved. However, the cancellations are exact only up to at most next-to-leading logarithmic order. This leaves some unavoidable residual dependence, which can be used to tune the procedure and obtain optimal agreement with data.

In SHERPA the CKKW merging of matrix elements and parton showers is accomplished as follows:

1. All cross sections $\sigma_{k}$ for processes with $k=0,1, \ldots, N$ extra partons are calculated with the constraint that the matrix-element final states pass the jet criteria. They are determined by a $k_{T}$ measure and the minimal distance is set by the actual merging scale $Q_{\text {cut }}$. Beyond that, $Q_{\text {cut }}$ also acts as a regulator setting the factorisation $(\mathrm{PDF})$ as well as the renormalisation $\left(\alpha_{s}\right)$ scales of the matrix-element calculations. The $k_{T}$ measure used for jet identification in electron-positron collisions can be written as [97]

$$
Q_{i j}^{2}=2 \min \left\{E_{i}^{2}, E_{j}^{2}\right\}\left(1-\cos \theta_{i j}\right),
$$

and quantifies the $k_{T}$ distance between the final-state particles $i$ and $j$. The jet cuts are satisfied if $Q_{i j}>Q_{\text {cut }}$. For hadron-hadron collisions, a $k_{T}$ scheme is employed, which defines two final-state particles to belong to two different jets, if their relative transverse momentum squared, defined as

$$
Q_{i j}^{2}=2 \min \left\{m_{T}^{(i)}, m_{T}^{(j)}\right\}^{2} \frac{\cosh \left(y^{(i)}-y^{(j)}\right)-\cos \left(\phi^{(i)}-\phi^{(j)}\right)}{D^{2}}
$$

is larger than the critical value $Q_{\text {cut }}^{2}$. In addition, the transverse momentum of each jet has to be larger than the merging scale $Q_{\text {cut }}$. The magnitude $D$, which is of order 1 , is a parameter of the jet algorithm, see [98]. 
2. Processes of fixed parton multiplicity are chosen with probability $\sigma_{k} / \sum_{l=0}^{N} \sigma_{l}$. The event's hard process is picked from the list of partonic processes having the desired multiplicity and according to their particular cross-section contributions. All particle momenta are distributed respecting the correlations encoded in the matrix elements. Merged samples therefore fully include lepton-jet and jet-jet correlations up to $N$ extra jets.

3. The parton configuration of the matrix element has to be analysed to eventually accomplish the reweighting. The partons are clustered backwards according to the same $k_{T}$ jet clustering algorithm used for the regularisation of the final-state phase space of the matrix elements (cf. step 1). The clustering is guided by the physically allowed parton combinations and automatically yields the nodal $k_{T}$ values $Q_{i j}$ of each parton emission. It is stopped after a $2 \rightarrow 2$ configuration (a core process) has been identified. In fact, this backward clustering constructs a limit of leading logarithmic accuracy of the full radiation pattern, i.e. determines a possible parton-shower history. The sequence of clusterings can thus be taken as a pseudo shower configuration, off which the event's evolution will be properly continued by parton showering.

4. The reweighting proceeds according to the reconstructed shower history. For the strong-coupling weight, the identified nodal $k_{T}$ values are taken as scales in the strong-coupling constants and replace the predefined choice of the initial generation. The Sudakov weight attached to the matrix elements accounts for having no further radiation resolvable at $Q_{\text {cut }}$. The NLL Sudakov form factors employed, cf. [97], are defined by

$$
\begin{aligned}
& \Delta_{q}\left(Q, Q_{0}\right)=\exp \left\{-\int_{Q_{0}}^{Q} d q \Gamma_{q}(Q, q)\right\}, \\
& \Delta_{g}\left(Q, Q_{0}\right)=\exp \left\{-\int_{Q_{0}}^{Q} d q\left[\Gamma_{g}(Q, q)+\Gamma_{f}(q)\right]\right\},
\end{aligned}
$$

where $\Gamma_{q, g, f}$ are the integrated splitting functions for $q \rightarrow q g, g \rightarrow g g$ and $g \rightarrow q \bar{q}(f \bar{f})$, given by

$$
\begin{aligned}
\Gamma_{q}(Q, q) & =\frac{2 C_{F}}{\pi} \frac{\alpha_{s}(q)}{q}\left(\ln \frac{Q}{q}-\frac{3}{4}\right), \\
\Gamma_{g}(Q, q) & =\frac{2 C_{A}}{\pi} \frac{\alpha_{s}(q)}{q}\left(\ln \frac{Q}{q}-\frac{11}{12}\right), \\
\Gamma_{f}(q) & =\frac{N_{f}}{3 \pi} \frac{\alpha_{s}(q)}{q},
\end{aligned}
$$

respectively. $\Gamma(Q, q)$ is cut off at zero, such that $\Delta_{q, g}\left(Q, Q_{0}\right)$ retains its probability interpretation for having no emission resolvable at scale $Q_{0}$ during the evolution from $Q$ to $Q_{0}$. Hence, $\Delta$-factors are used to reweight in accordance to the appearance of external parton lines. The ratio of two Sudakov form factors $\Delta\left(Q, Q_{0}\right) / \Delta\left(q, Q_{0}\right)$ accounts for the probability of having no emission resolvable at $Q_{0}$ during the evolution from $Q$ to $q$. Ratio factors thus are employed for the reweighting of internal parton lines. In both cases the lower limit is taken to be $Q_{0}=Q_{\text {cut }}$ or $Q_{0}=D Q_{\text {cut }}$ for partons that are clustered to a beam or to another final-state parton, respectively.

5. After the reweighting of the matrix-element configuration, the parton shower is invoked starting from the pseudo history constructed before. Each parton continues its evolution at the scale where it was produced. For the virtuality-ordered parton shower of APACIC++, this scale is given by the invariant mass of the mother parton belonging to the QCD splitting, through which the considered parton has been initially formed. Recall that this QCD splitting is identified by the "clusterbackwards" procedure. For the cases where the considered leg originates from the core process, the (hard) scale still needs to be determined. For example, all four partons resulting from a clustering that terminated in a pure QCD $2 \rightarrow 2$ process will commence their evolution at the corresponding hard QCD scale. 

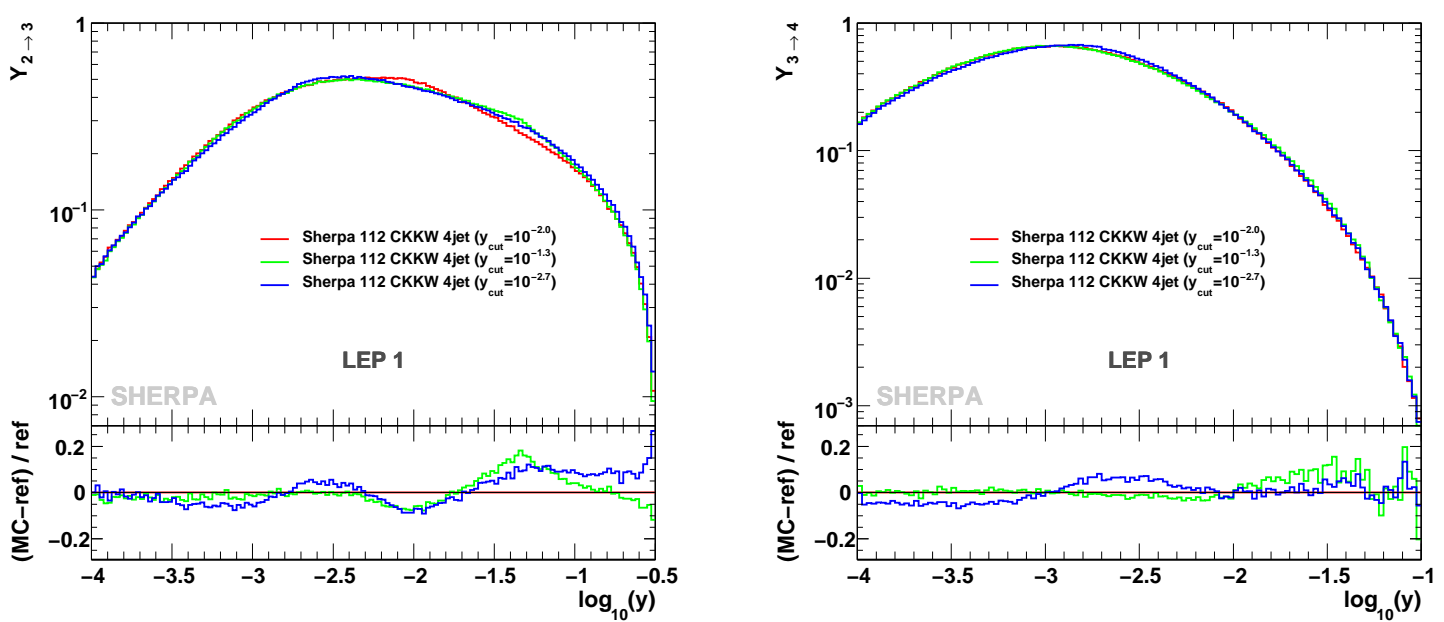

Fig. 8 Durham differential $2 \rightarrow 3$ (left) and $3 \rightarrow 4$ (right) jet rates as a function of the jet-resolution parameter $y$ for different merging-scale choices $y_{\text {cut }}$.

6. In all circumstances parton-shower radiation is subject to the condition that no extra jet is produced. Any emission that turns out to be harder than the separation cut $Q_{\text {cut }}$ is vetoed. The exception to this veto - called highest-multiplicity treatment - is for matrix-element configurations with the maximal number $N$ of extra partons. These cases require the parton shower to cover the phase space for more jets than those produced by the matrix elements. To obtain an inclusive $N$-jet prediction, the veto therefore is on parton emissions at scales harder than the softest clustering scale, $Q_{\text {softest }} \geq Q_{\text {cut }}$. Of course, correlations including the $N+1$ st jet are such only approximately taken into account.

As a final remark it is worthwhile to note that the procedure described here has been fully automated, i.e. in SHERPA the merging of the different jet multiplicities is handled on the fly.

\subsection{Applications}

\subsection{1 $\quad e^{+} e^{-} \rightarrow$ hadrons}

To exemplify the working of the algorithm described above one may consider the simple example of jet production in electron-positron annihilation.

The lowest-order matrix elements account for the $k=2$ processes $e^{+} e^{-} \rightarrow q \bar{q}$. They all have the same topology and the corresponding Sudakov weights read

$$
W_{2}=\left[\Delta_{q}\left(E_{\text {cm }}, Q_{\text {cut }}\right)\right]^{2} .
$$

The vetoed parton showers for both the quark and the antiquark start at the scale equal to the centreof-mass energy $E_{\mathrm{cm}}$ of the $e^{+} e^{-}$annihilation. A veto is applied for emissions above $Q_{\text {cut }}$. There are two topologies for the $k=3$ subprocesses, i.e. for $e^{+} e^{-} \rightarrow q \bar{q} g$, corresponding to the emission of a gluon off the quark or antiquark, respectively. The related Sudakov weights read

$$
\begin{aligned}
W_{3} & =\Delta_{q}\left(E_{\mathrm{cm}}, Q_{\mathrm{cut}}\right) \frac{\Delta_{q}\left(E_{\mathrm{cm}}, Q_{\mathrm{cut}}\right)}{\Delta_{q}\left(q, Q_{\mathrm{cut}}\right)} \Delta_{q}\left(q, Q_{\mathrm{cut}}\right) \Delta_{g}\left(q, Q_{\mathrm{cut}}\right) \\
& =\left[\Delta_{q}\left(E_{\mathrm{cm}}, Q_{\mathrm{cut}}\right)\right]^{2} \Delta_{g}\left(q, Q_{\mathrm{cut}}\right),
\end{aligned}
$$

where $q$ denotes the scale at which the gluon has been resolved. Again the parton showers for the quark and antiquark start at $E_{\mathrm{cm}}$. The shower for the gluon is initiated at scale $q$. All showers are subject to a veto on $Q_{\text {cut }}$. Note that in the case $N=k=3$ the weight reduces to

$$
W_{3}=\left[\Delta_{q}\left(E_{\mathrm{cm}}, q\right)\right]^{2}
$$



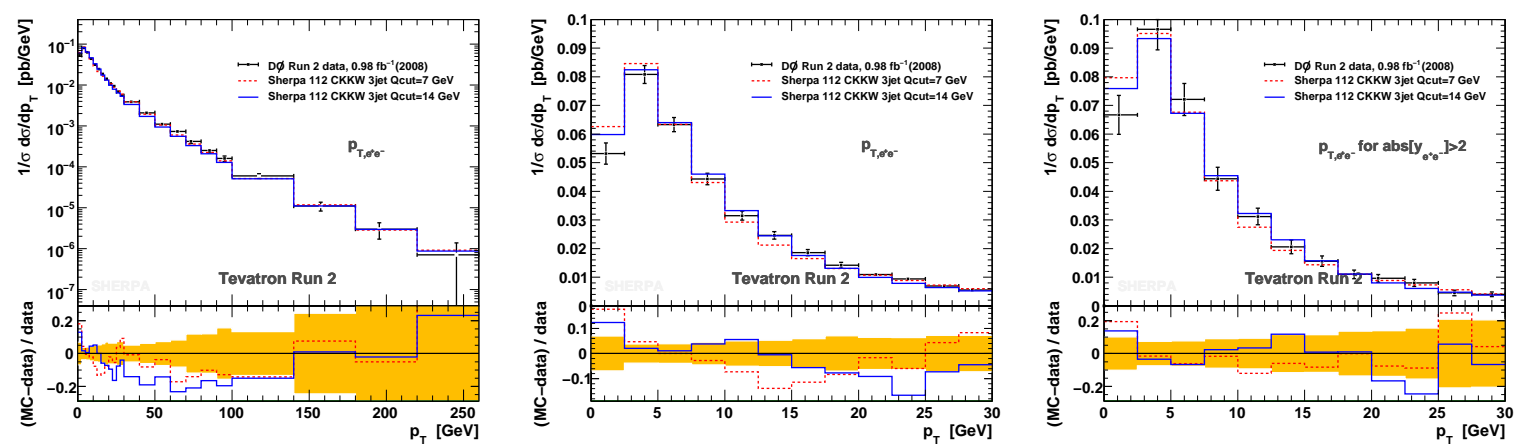

Fig. 9 SHERPA CKKW shape predictions for two different merging-scale choices and various $p_{T}$ spectra of the vector boson in $Z+$ jets production at Tevatron Run II. The left part shows the distribution for the full $p_{T}$ range as measured by D $\varnothing$, whereas the other parts focus on the peak region. The rightmost plot has been obtained by requiring forward-rapidity lepton pairs. Data are taken from [99] and the shaded bands visualize the sum of statistical and systematic uncertainties on the data.

and shower vetoes are performed w.r.t. $q$.

The quality of the procedure can properly be studied by means of the differential jet rates, which show the distributions of $y=Q^{2} / S$ values at which an $n+1$ jet event is merged into an $n$ jet event according to the jet clustering scheme employed by the algorithm. In particular they allow a thorough inspection of the transition region $y \approx y_{\text {cut }}$. The merging procedure provides a good description of both the hard and the soft part of these distributions. Large values of $y$ indicate the region of hard emissions, where the matrix elements improve over any given parton-shower estimate. For decreasing values of $y$, the real-emission matrix elements diverge and descriptions based on them become unreliable. Instead kinematically enhanced logarithms need to be resummed to all orders as accomplished by parton showers. Shower-level results as given by the procedure for the Durham differential $n \rightarrow n+1$ jet rates are depicted in Fig. 8 for $n=2,3$. They clearly show the features described above: predictions for different values of $y_{\text {cut }}$ vary at a level of $15 \%$ only. The transitions between shower and matrix-element domains for the various $y_{\text {cut }}$ choices are smooth. The plots emphasise that the merging procedure works well and at the level of accuracy it claims to provide for the parton-shower tree-level matrix-element matching.

\subsection{2 $W / Z+$ jets}

Weak boson production in association with jets is one of the most prominent backgrounds in many new-physics searches with signals of the type multijets plus missing transverse energy plus leptons. The validation and tuning of Monte Carlo tools against newest Tevatron data on weak boson plus jets production therefore has to be considered a crucial prerequisite for successfully applying the available tools in LHC analyses. The extrapolation to higher energies can only be more meaningful once it starts off a solid and well understood test scenario. With more and more Tevatron data coming in, its discriminating power gradually increases and data can thus be used to refine the multijet-production algorithms, even rule out choices or falsify assumptions that have been made in constructing them.

Here, new comparisons are presented using very recent experimental $W / Z+$ jets data taken during Run II at the Fermilab Tevatron and published by the D $\varnothing$ and CDF collaborations in [99] and $[92,100]$, respectively. All CKKW predictions in this subsection have been obtained from inclusive samples where matrix elements including up to three extra (light-flavour) partons were merged with the parton showers. The PDF set used was CTEQ6L. Note that effects of the underlying event and hadronisation have not been taken into account for this study.

Figure 9 shows transverse momentum distributions of the Drell-Yan lepton pair. On a rather inclusive level these spectra allow for insight to the nature of the occuring QCD radiation, which the boson is finally recoiling against. The data are taken from a $\mathrm{D} \varnothing$ shape measurement of $Z$ boson events with mass $40<M<200 \mathrm{GeV}$ and integrated luminosity of almost $1 \mathrm{fb}^{-1}$ [99]. The overall agreement is satisfactory. In the left panel of Fig. 9 the full $p_{T}$ range measured up to $260 \mathrm{GeV}$ is depicted. The two CKKW predictions differing in their choice of the merging scale $Q_{\text {cut }}$ tend to undershoot the data 

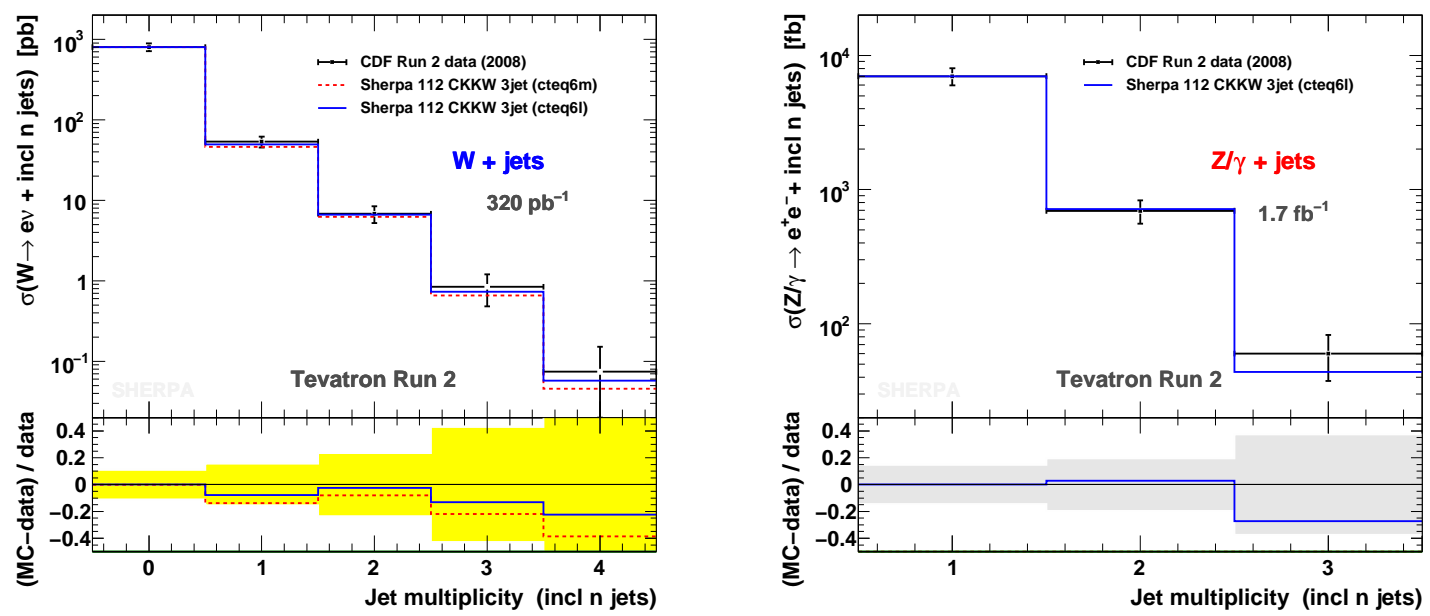

Fig. 10 Inclusive $n$ jet cross sections compared to CDF Tevatron Run II data for $W+$ jets production [92] (left panel) and $Z+$ jets production [100] (right panel). The shaded bands represent the full error on the data, except for the luminosity error being not included for $W+$ jets. In both cases the blue solid line labels SHERPA CKKW predictions obtained with the CTEQ6L PDF set; for the red dashed curve, the CTEQ6M set was used. The predictions for $W$ and $Z+$ jets are normalized to the inclusive $n=0$ and $n=1$ cross sections, resulting in constant $K$-factors of 1.33 (1.14 for the dashed curve) and 1.71, respectively. For cuts and details of both analyses, the reader is referred to the respective publication.

between $30<p_{T}<80 \mathrm{GeV}$. This may be improved further by tuning the merging scale and fully including underlying-event plus hadronisation effects. The center and right panels respectively show the region of soft $p_{T}$ without and with the additional constraint of reconstructing the rapidity of the Drell-Yan pair to be larger than 2. For both plots, the results in the very first bin may be corrected by tuning the intrinsic $k_{T}$ smearing parameters. The good agreement, in particular for forward lepton-pairs events, confirms that resummation effects are reasonably accounted for.

The TeV centre-of-mass energies of the Tevatron/LHC provide sufficiently/enormously large phase space to be filled by relatively hard QCD radiation giving rise to (hard) jets accompanying the vector boson. Such topologies cannot be generated by parton showers. Figure 10 displays inclusive cross sections for various jet multiplicities. These cross sections are very sensitive to the correct description of QCD multijet final states and hence provide excellent probes for SHERPA's merging approach. Figure 11 shows the transverse momentum spectra of jets produced in association with the Drell-Yan lepton pairs. These observables allow a more detailed study of the QCD structure of the events. In both figures CKKW predictions have been compared to CDF data published in [92] for $W+$ jets and [100] for $Z / \gamma^{*}+$ jets production. ${ }^{5}$ The predictions are normalized by a constant $K$-factor to the lowest available $n$ jet cross sections, $n=n_{0}$. The description of the $n>n_{0}$ cross sections then is very satisfactory and the predictions are low for the highest jet only. Merging in matrix elements with even more extra partons will certainly improve on this situation. The same constant $K$-factors were employed to generate the jet $p_{T}$ spectra. As a result rate and shape of all $p_{T}$ distributions is in quite remarkable agreement with the data. There is a tendency of overestimating the hardness of the leading jet, yet the predictions are consistent with data. For the case of $W+$ jets production, a second CKKW curve was added obtained by employing the CTEQ6M PDF set. This led to a smaller $K$-factor and softer spectra in general. It also provides an estimate of the uncertainty that comes along with the use of different PDF sets.

A clear deficiency of the parton shower is the limitation in correctly accounting for boson-jet (leptonjet) and jet-jet correlations. This drawback can be overcome by using the CKKW merging approach. Examples for $Z / \gamma^{*}+X$ production at the LHC are given in Fig. 12. The left panel depicts the pseudorapidity difference between the lepton pair arising from the decay of the vector boson and the hardest jet

\footnotetext{
${ }^{5}$ The new data has been used to further validate and tune SHERPA by revising and finally changing some of the default choices of the implemented merging procedure; the new settings will be made available to the public.
} 

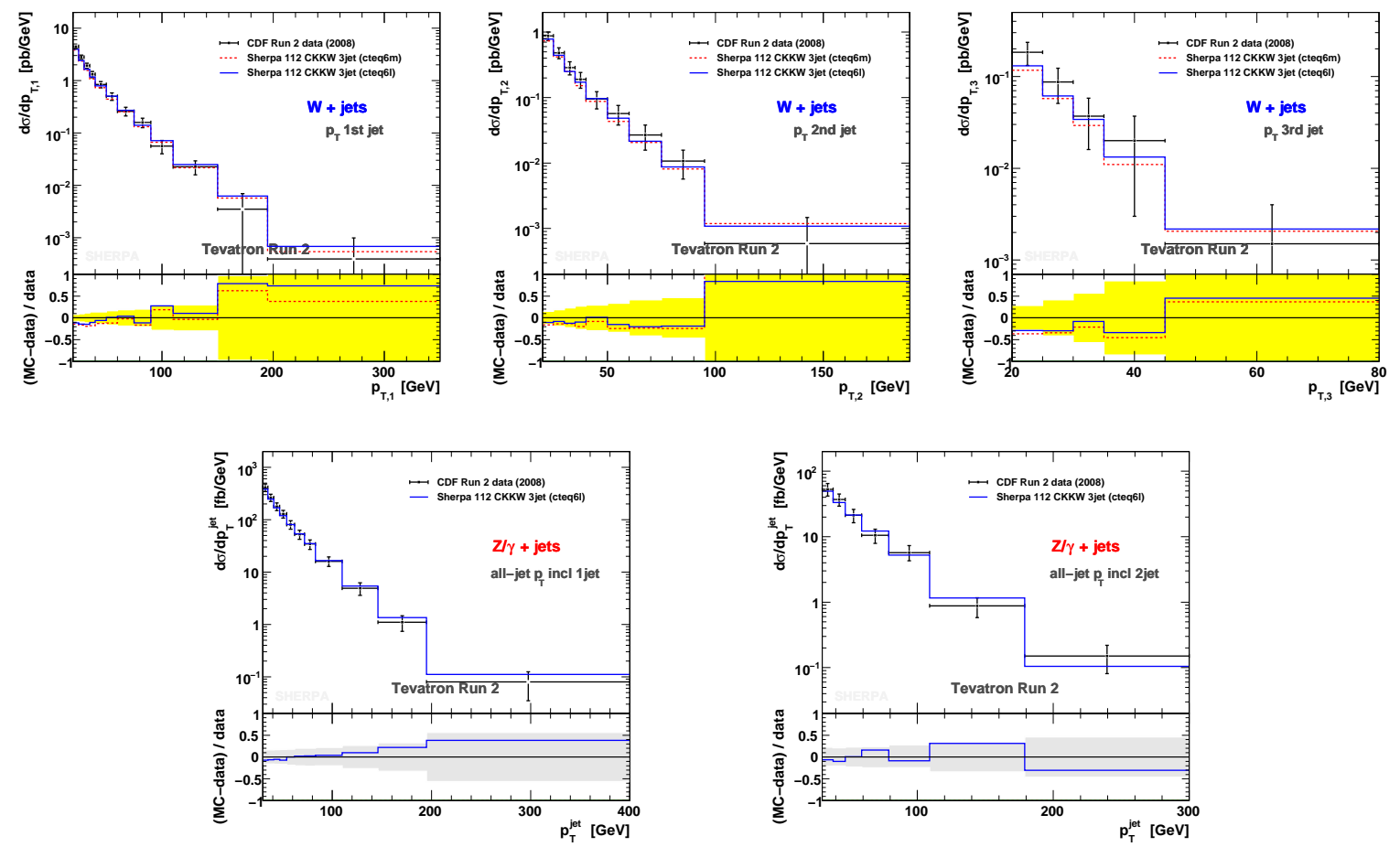

Fig. 11 Jet $p_{T}$ spectra compared to CDF Tevatron Run II data for $W+$ jets production [92] (top row) and $Z+$ jets production [100] (bottom row). Labelling, $K$-factors and cuts are the same as given in the caption of Fig. 10. Note that the top row gives inclusive jet $p_{T}$ spectra of the $n$th jet; the bottom row depicts all-jet $p_{T}$ spectra for events with at least $n$ jets, i.e. a 3 jet event gives 3 entries to all distributions up to $n=3$.

accompanying the boson. The prediction obtained by solely adding parton showering to the Drell-Yan lepton-pair production (here given by the APACIC++ shower) shows a suppression at low $|\Delta \eta|$. The correct correlation is encoded in the QCD real-emission matrix elements for $Z / \gamma^{*}$ production and can hence be accounted for by the CKKW approach. As a consequence the suppression has disappeared in the CKKW merging prediction. The distributions of the spatial separation between the second and third hardest jet are given in the right panel of Fig. 12. The pure shower approach predicts separations that are too wide. This again is corrected for in the CKKW procedure by including matrix elements here up to three extra parton emissions.

As demonstrated by these examples CKKW predictions lead to considerable improvements where the application of parton showers merely gives approximations or is even misleading. An improved ISR description as well as a correct treatment of correlations involving jets can only be achieved by including the corresponding matrix elements for extra QCD radiation. Merging algorithms are therefore an ideal approach to provide inclusive $W / Z+$ jets samples up to a certain number of jets including effects of hard ISR and jet correlations. The comparison to recent Tevatron data proves the relevance of SHERPA's merging approach in describing multijet events realistically.

\subsection{3 $t \bar{t}+$ jets}

It is worthwhile to study the implications of the CKKW formalism for relevant processes at future colliders. An example for such a process, which plays a significant role, both as a signal for a better measurement of Standard Model parameters and as a background to new physics searches, is top quark pair production. In this publication it serves as an example for the merging of matrix elements and parton showers in processes with strongly interacting heavy resonances. Properly implemented real next-to-leading order corrections can be crucial for this kind of interaction, as pointed out e.g. in [70]. The combination of matrix elements and parton showers in this respect is based on using a (potentially Breit-Wigner improved) narrow width approximation to compute the corresponding hard matrix 

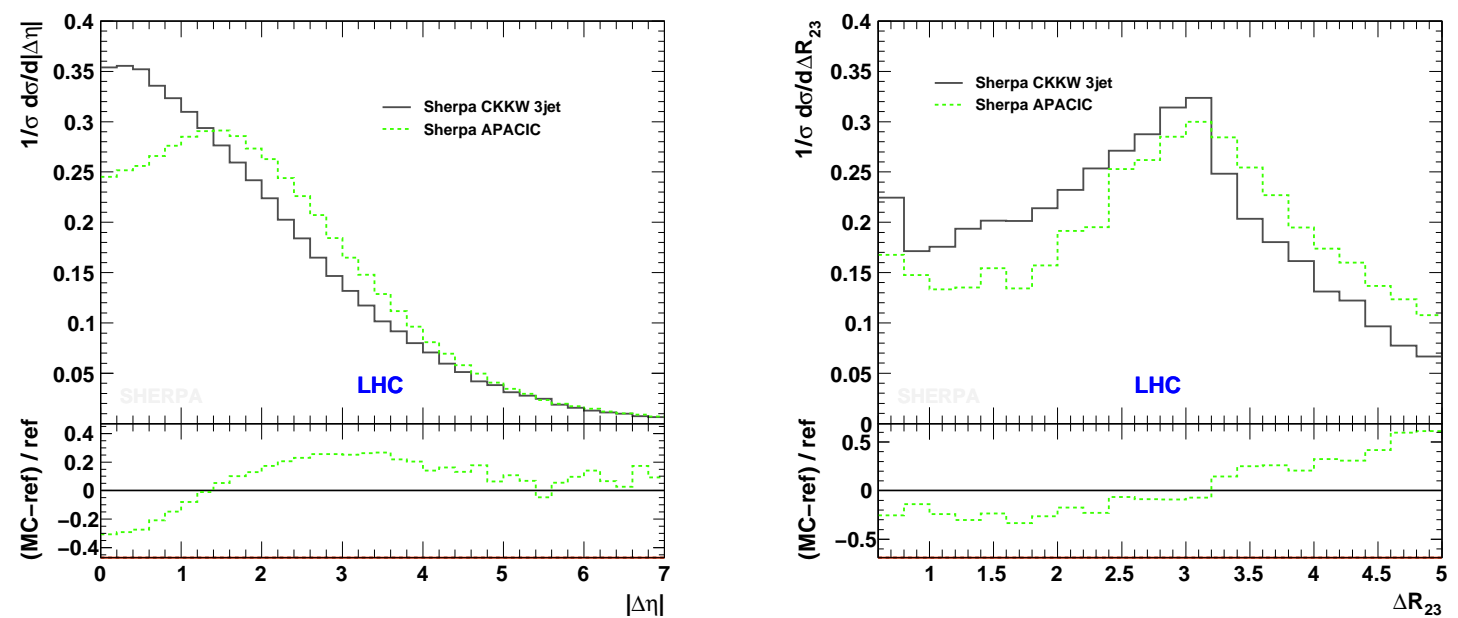

Fig. 12 Left panel: $|\Delta \eta|$ distributions between the vector boson and the first jet in $Z / \gamma^{*}+$ jets production at the LHC. Right panel: Spatial separation between the second and third hardest jet, $\Delta R_{23}$, in $Z / \gamma^{*}+$ jets production at the LHC. Dark solid curves represent CKKW predictions including matrix elements up to three extra partons. The bright dashed curves are pure shower predictions generated by APACIC++. Jets are defined by the Run II $k_{\perp}$-algorithm with parameter $D=0.4$ [98], and the cuts are $p_{T}^{\text {jet }}>20 \mathrm{GeV}$ and $\left|\eta^{\text {jet }}\right|<4.5$.

elements, cf. Sec. 3. Through the identification of the decaying intermediate particle, a parton shower can independently be assigned to its production and decay. Upon application of the CKKW merging algorithm and when integrating over the phase space of the outgoing particles, jet measures $Q$ between strongly interacting particles must be larger than a critical value $Q_{\text {cut }}$. Due to the factorised structure of the process, this critical value might be chosen separately per subprocess, i.e. there may be different values $Q_{\text {prod }}=Q_{\text {cut }}(\operatorname{prod})$ and $Q_{\text {dec }, i}=Q_{\text {cut }}\left(\operatorname{dec}_{i}\right)$.

Technically the merging proceeds as follows: Once a particular momentum configuration is chosen for the hard matrix element, the parton-shower history is identified as in the standard merging prescription. Since the full amplitude factorises over time-like propagators, only particles belonging to the same subamplitudes $\mathcal{A}_{\text {prod }}$ or $\mathcal{A}_{\mathrm{dec}, i}$ can be combined. When reweighting the hard matrix element with Sudakov form factors, the potentially different cutoff values $Q_{\text {cut }}$ must be employed. Sudakov reweighting for the resonant intermediate particles takes places according to the original prescription. The jet veto is applied separately within each part of the parton showers related to a different subamplitude, employing the corresponding veto scale $Q_{\text {cut }}$. The highest multiplicity treatment is applied separately in each subamplitude. To summarise, the above prescription translates into the CKKW method being applied separately and completely independent for each part of the process under consideration.

For this procedure, reweighting with analytic Sudakov form factors for heavy quarks is necessary. It must therefore be assured that those match the respective distributions generated by the parton shower. A corresponding comparison is shown in Fig. 13. Predictions from APACIC++ and an analytic calculation performed in [101] are displayed for the Durham $k_{T}$-jet rates at a fictious electron positron collider operating at $500 \mathrm{GeV}$.

Figure 14 shows the impact of the description of additional hard radiation through appropriate matrix elements in the case of $t \bar{t}$-production at the LHC. Subsamples from a given matrix element configuration are displayed in colour with the colour code explained above the figure. The following notation is employed

- The first number denotes the additional jet multiplicity in the $t \bar{t}$ production process.

- The second number denotes the additional jet multiplicity in the $t$ and $\bar{t}$ decay process.

It can be observed that the transverse momentum spectrum of the produced heavy quark pair is significantly enhanced at high values when employing the CKKW prescription. This is easily explained by the failure of the parton-shower approach to correctly describe hard initial-state radiation. Also, it is clearly 


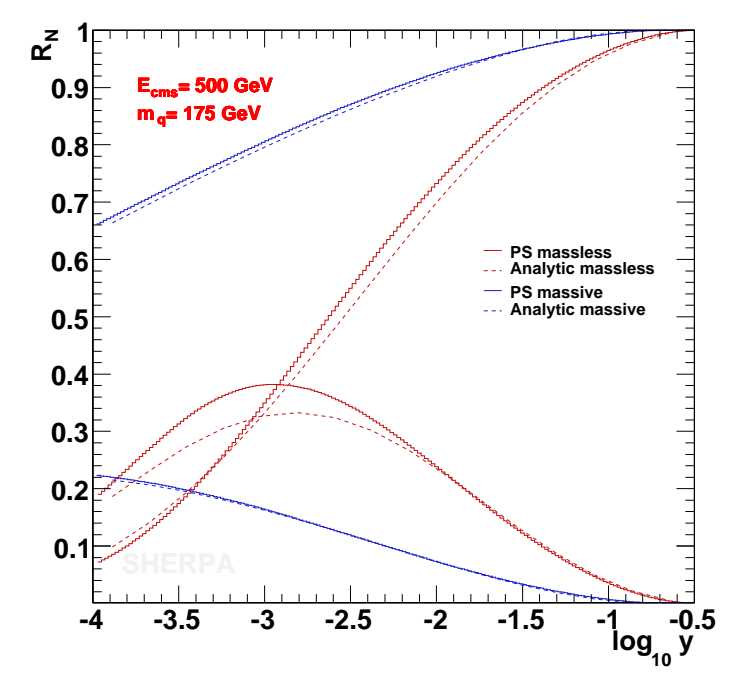

Fig. 13 Comparison of the Durham 2- and 3-jet rates for massive quarks, calculated according to [101], with results from APACIC++. Good agreement is found between the parton-shower result (solid histogram) and the analytical calculation (dashed line). For reference, results with massless quarks are also shown.

seen that subsamples including an additional hard jet in the production process (1-0 and 1-1) deform the pseudorapidity spectrum of the first additional hard jet (identified through $k_{T}$-clustering and Monte Carlo truth based $b$-tagging). The pure parton-shower approach predicts a dip at central rapidity, while the merged sample does not show this feature.

\section{Multiple parton interactions}

The hardest (primary) partonic interaction in hadronic collisions may be accompanied by softer (secondary) ones, which may, however, still fall in the realm of perturbative QCD. This is a valid assumption and clear evidence has come from experimental studies carried out by the CDF collaboration at the Fermilab Tevatron during Run I and Run II $[102,103]$. It was shown that a correct description at the hadron level of particle multiplicities and jet activities can only be achieved by Monte Carlo event generators that incorporate a model for multiple parton scatterings.

The basic idea of such a model is to postulate the probability distribution for the occurrence of multiple scatterings. According to the model of [35] this is given as a function of the non-diffractive cross section $\sigma_{\mathrm{ND}}$, the hard perturbative cross section $\sigma_{\text {hard }}$ and the outgoing transverse momentum $p_{\perp}$ in the scattering by

$$
p\left(p_{\perp}, b\right)=f_{c} f(b) \frac{1}{\sigma_{\mathrm{ND}}} \frac{\mathrm{d} \sigma_{\mathrm{hard}}}{\mathrm{d} p_{\perp}},
$$

where the prefactors $f_{c}$ (normalisation) and $f(b)$ (proton shape function) incorporate an additional impact-parameter dependence of the distribution on an event-by-event basis. In the collinear factorisation approach a phase-space cut on the hard matrix elements, e.g. a minimum jet resolution cut $Q_{\text {cut }}$, has to be introduced to obtain a well-defined differential cross section in perturbation theory. Then $\mathrm{d} \sigma_{\text {hard }} / \mathrm{d} p_{\perp}$ receives its dominant contributions from $2 \rightarrow 2$ processes and, hence, the definition of $p_{\perp}$ in Eq. (31) is unambiguous. Moreover it suffices to consider hard $2 \rightarrow 2$ QCD processes only.

The multiple-interactions model of SHERPA is implemented in the module AMISIC++, which generates multiple scatterings according to the basic ideas listed above. There are however important details where the SHERPA approach deviates from the original formalism presented in [35]. Firstly, all secondary interactions are undergoing parton-shower corrections and, therefore, AMISIC++ has been interfaced to the parton-shower module APACIC++. A second class of extensions concerns the combination of the CKKW merging approach with the modelling of the multiple interactions, which essentially are independent of 

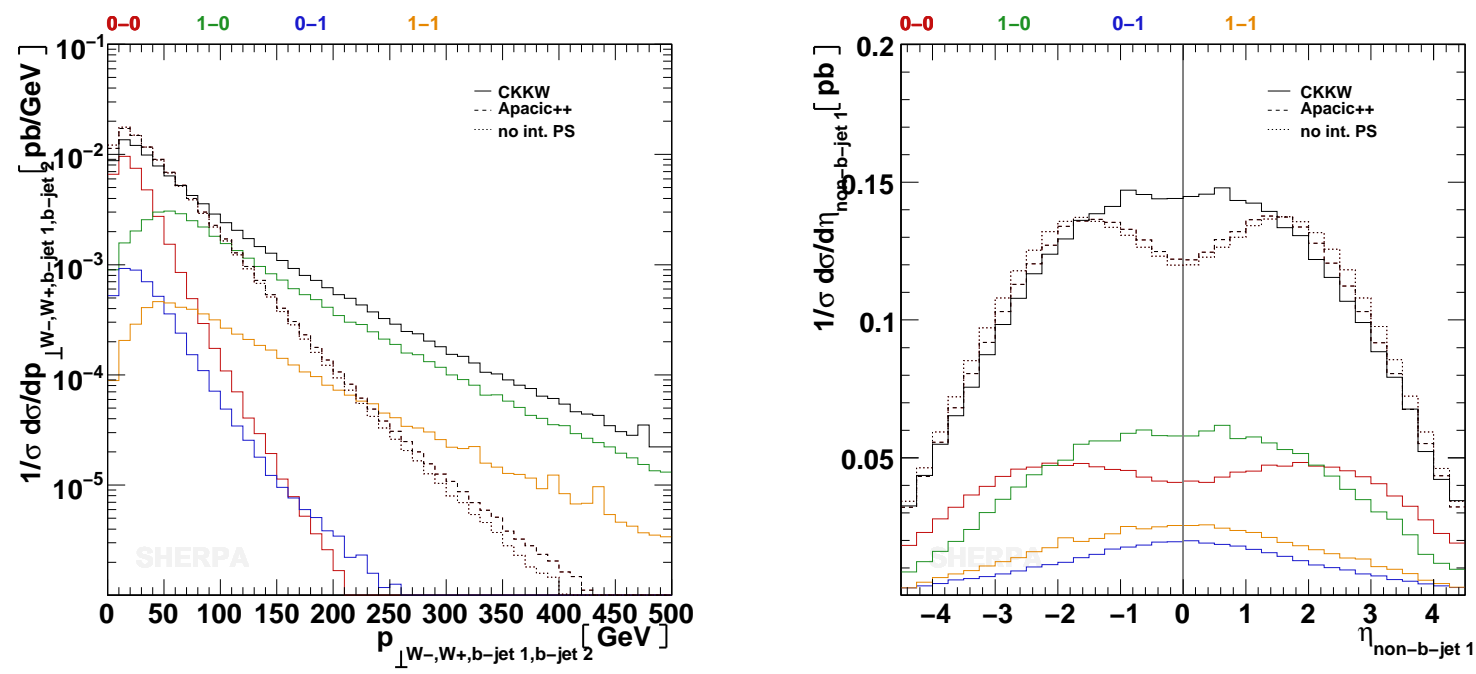

Fig. 14 Transverse momentum spectrum of the $t \bar{t}$-pair (left panel) and rapidity spectrum of the first extra jet (right panel) in $p p \rightarrow t \bar{t} \rightarrow W^{+} W^{-} b \bar{b}+$ jets events at $\sqrt{s}=14 \mathrm{TeV}$. Shown is a comparison between the pure parton-shower result (no int. PS), parton shower with radiation off intermediate top quarks (Apacic++) and a CKKW-merged sample. Contributions from various matrix element configurations are highlighted in colour for the merged sample, with the colour code indicating the additional jet multiplicity in the production $\otimes$ decay process. Up to one extra jet has been simulated through matrix elements in the production and each decay.

the treatment of the hardest scattering in the collision. It is vital that the parton showers related to secondary interactions respect the initial $p_{\perp}$ distribution of the hard scatterings. In particular, partons with a $k_{T}$-separation from other partons larger than $p_{\perp}$ of the respective (secondary) interaction must not be radiated. The appropriate way to incorporate this constraint into the parton-shower formalism is in fact identical to the realisation of the highest-multiplicity treatment in the CKKW approach. The corresponding algorithm then works as follows:

1. Create a kinematic configuration of the final-state particles according to the hard matrix element of the primary interaction and run its parton-shower evolution according to the CKKW formalism (cf. Sec. 5).

2. Employ a $k_{T}$-type algorithm in the $E$-scheme to cluster the complete final state of the previous scattering into a $2 \rightarrow 2$ process, similar to how this is done to define starting conditions for showers in the CKKW approach. Set the starting scale for multiple interaction evolution to the maximum of

(a) the largest relative transverse momentum between QCD partons that have been combined, or

(b) the transverse momentum in the $2 \rightarrow 2$ process, if this process contains a QCD parton in the final state.

3. Select the $p_{\perp}$ of the next secondary interaction according to Eq. (31). If this happens for the first time in the event, select the impact parameter $b$ of the collision as described in [35]. Create the kinematic configuration of the secondary interaction.

4. Set the jet veto scale of the parton shower to the transverse momentum selected in step 3. Start the parton shower of the secondary interaction at the QCD scale

$$
\mu_{\mathrm{QCD}}^{2}=\frac{2 s t u}{s^{2}+t^{2}+u^{2}} .
$$

5. Return to step 2 . 

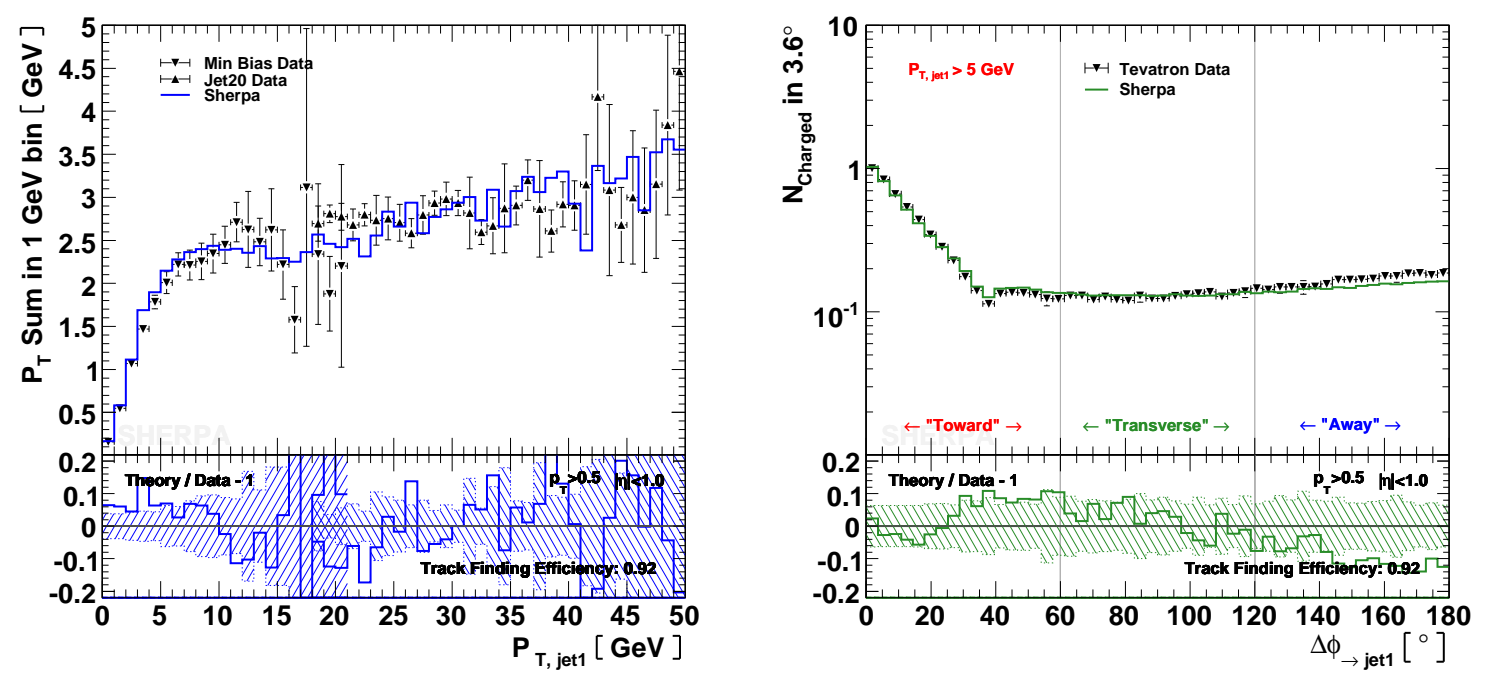

Fig. 15 The left panel displays the average scalar $p_{T}$ sum versus $p_{T}$ of the leading charged particle jet while the right panel displays the average charged particle multiplicity versus $\Delta \phi$ with respect to the leading charged particle jet for $p_{T}>5 \mathrm{GeV}$ of this jet. In both cases Sherpa results are contrasted with data from [103].

The above algorithm works for pure QCD hard matrix elements as well as for electroweak processes being the primary hard scattering in the event. In the QCD case the selected starting scale for the determination of the first additional interaction reduces to the transverse momentum in Eq. (31) and is thus equal to the original ordering parameter. For electroweak core processes, e.g. $W$ or $Z$ boson production, there is no such unique identification. However, it is required that the multiple scatterings in the underlying event do not spoil the jet topologies generated by the primary hard scattering. Regarding the electroweak bosons as being radiated off QCD partons during the parton shower evolution of a hard QCD event, it is appropriate to reinterpret this hard matrix element as a $\mathrm{QCD}+\mathrm{EW}$ process, whereof the simplest is an EW+1-jet process. In this way all primary jet topologies can be preserved.

An appropriate method to include an impact-parameter dependence in the multiple-interactions model is described in [35]. In AMISIC++ the default (anti-)proton shape in position space is chosen to follow a Gaussian matter distribution. AMISIC++ has primarily been validated using the data presented in [103]. These tests revealed that effects owing to the variation of the particular shape function are of minor importance for the description of the underlying event. It might however turn out that the shape function becomes more important when facing more precise data.

Finally, it has to be stressed that AMISIC++ relies on complete factorisation of the hard (primary) process and the additional scatterings in the underlying event. Corrections, which arise from multi-parton PDFs as discussed in [104], are not taken into account.

In Figure 15 predictions from SHERPA are compared with experimental data from [103]. Both observables are very sensitive to the correct description of the underlying event.

\section{Parton-to-hadron fragmentation}

After the parton shower has terminated, a configuration of coloured partons at some low scale of the order of a few $\mathrm{GeV}$ in transverse momentum emerges. These partons, in order to match experiments, have to be translated into hadrons. Since there is no first-principles approach yielding quantitative results, hadronisation is achieved by phenomenological models only. Usually, they are based on some qualitative ideas on how the parton-to-hadron transition proceeds, like, e.g., local parton-hadron duality or infrared safety, defining the model's coarse properties. However, many of the important finer details, often related to how flavour is created and distributed in the procedure, are entirely undefined and subject to phenomenological parameters only. These are essentially free and must be fixed by extensive 
comparisons with data, as done for instance in [105].

\subsection{Beam-remnant handling}

In collisions involving hadronic initial states, there is the additional problem that one or more final-state particles will be colour-connected to the hadron remnants. The breakup of the initial hadron into partons then needs to be modelled suitably. This is achieved in such a way that only a minimal set of particles (quarks and diquarks, the latter as carriers of baryon number) is produced in order to reconstruct the constituent flavour configuration of the corresponding hadron. Once their colour connections are established, the complete set of final-state partons can be hadronised affecting energy flows and other, similar properties of the event at hadron level.

The distribution of colour in the hadron remnants cannot be inferred from first principles. Hence phenomenological models have to be employed. In SHERPA the default distribution of colour is guided by the idea of minimising the relative transverse momentum of colour dipoles spanned by outgoing partons. When including multiple parton interactions in the simulation, it is not always possible to accomplish free colour selection in the hard process and minimisation of relative transverse momenta simultaneously. In such cases the colour configurations of the matrix elements are kept but the configuration of the beam remnants is shuffled at random until a suitable solution is found. The shuffling is implemented also as an alternative choice if no multiple interactions are present. So far, no significant differences have been found in the predictions from the two models on the level of physical observables.

In addition to the issues related to colour neutralisation with the beam remnants, all shower initiators and beam partons obtain a mild $k_{\perp}$-kick according to a Gaussian distribution parametrising the Fermi motion of these particles inside the incoming hadron. It should be stressed that within SHERPA mean and width of this distribution are rather small (about $0.2-0.8 \mathrm{GeV}$ ), such that the physical interpretation indeed holds.

\subsection{Cluster-hadronisation model}

For a long time, for the hadronisation of partons, SHERPA has relied on the implementation of the Lund string model [106] in PYTHIA, accessible through a corresponding interface. Since version 1.1, SHERPA employs its own module AHADIC [107], which implements a cluster-hadronisation model as described in [36] and extended by ideas presented in [37]. The basic assumption underlying this class of models is local parton-hadron duality, i.e. the idea that quantum numbers on the hadron level follow very closely the flow of quantum numbers on the parton level. In this framework, the mass spectrum of the emerging colour-neutral clusters is dominated by typical hadron masses or masses slightly above. It is therefore natural to think of them as some kind of "hadron matter", carrying the flavour and momentum quantum numbers of hadrons. This motivates to translate the light clusters directly into hadrons or, if they are too heavy, to treat them like hitherto unknown heavy hadron resonances, which decay further into lighter ones. The idea underlying AHADIC is to take this interpretation very literal, to compose clusters out of all possible flavours, including diquarks, and to have a flavour-dependent transition scale between clusters and hadrons. This results in solely translating the very light clusters directly into hadrons, whereas slightly heavier clusters experience a competition between being either translated into heavy hadrons or being decayed into lighter clusters, see below. In addition, for all decays, a QCD-inspired, dipole-like kinematics is chosen.

In more detail, in AHADIC, the hadronisation of quarks and gluons proceeds as follows:

First of all, the four-momenta of all partons are boosted and scaled such that all of them reside on constituent-mass shells $\left(m_{u}=m_{d}=0.3 \mathrm{GeV}, m_{s}=0.5 \mathrm{GeV}, m_{c}=1.8 \mathrm{GeV}, m_{b}=5.2 \mathrm{GeV}\right)$, whereas the gluon remains massless in contrast to the cluster model implemented in HERWIG and HERWIG++. In addition to the quarks, further states will emerge, diquarks, which effectively carry baryonic quantum numbers. Their masses are typically given by the sum of the constituent masses of the two respective quarks making them up, i.e. $m_{q q^{\prime}}=m_{q}+m_{q^{\prime}}$. In the following, quarks and diquarks, will commonly be called "flavours".

At the onset of hadronisation, in cluster models the gluons must decay. This is because in all hadronisation models, all hadrons are made of flavour constituents only. This forced decay is the reason, why an effective gluon mass enters as parameter in HERWIG and HERWIG++, where the gluon decays with no spectator involved. In AHADIC, the gluon's decay is facilitated in a dipole framework, and there will 

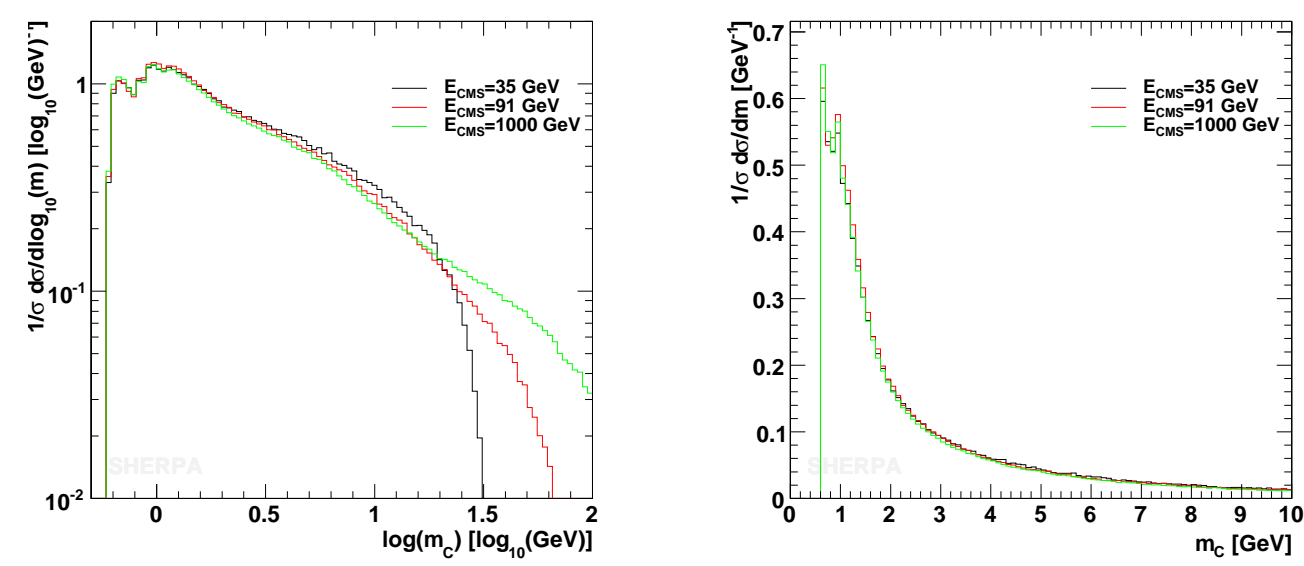

Fig. 16 Cluster-mass distribution in $e^{+} e^{-}$collisions at various center-of-mass energies.

always be a colour-connected spectator to compensate the recoil when the massless gluon decays into massive flavours. In these non-perturbative, enforced decays, the transverse momentum measure $p_{\perp}$ is bounded from above by a parameter $p_{\perp}^{\max }$, typically of the order of the parton-shower cut-off scale.

In addition, in AHADIC a non-perturbative $\alpha_{s}$ coupling has been encoded for all decays (gluons and clusters). It is chosen such that it agrees with a measurement from the GDH sum rule. The corresponding form given in [108] has been implemented with all parameters. In addition to this default setting, constant-value choices are also available.

For the production of the flavours, the available phase space leads to kinematic enhancements of lighterflavour pairs in dependence on the mass of the dipole (i.e. pair of gluon and spectator). It is supplemented by constant weight factors, $\mathcal{P}_{q}$, also used in cluster decays to hadrons. When all gluons have been decayed, colour-connected flavours are merged into colour-neutral clusters. In contrast to the original version of the cluster model, also diquarks are allowed as cluster constituents from the beginning such that in every stage of the hadronisation baryonic quantum numbers can be produced.

In order to guarantee the universality of this hadronisation procedure, it is important that the cluster mass distribution is almost independent of the centre-of-mass-energy in otherwise identical processes. This has been checked, e.g. for the case of $e^{+} e^{-}$annihilations into light quarks, cf. Fig. 16. The structures in the peak region appear owing to masses of the constituent quarks.

At every step, all clusters are checked whether they fall into one or both of two regimes, namely

- either the regime where the mass of the cluster is of the order of the mass of the heaviest hadron with a flavour wave component identical to the flavour content of the cluster, i.e. where $M_{c}<m_{h}+\delta_{h}$,

- or the regime where the mass of the cluster is lighter than the summed mass of the two heaviest hadrons it can decay into by adding a flavour-antiflavour pair to its flavour content, i.e. where $M_{c}<m_{h}+m_{h^{\prime}}+\delta_{h h^{\prime}}$.

In order to steer this classification further, two offsets $\delta_{h}$ and $\delta_{h h^{\prime}}$ have been introduced.

If a cluster is outside both regimes, it will decay further into two clusters. This proceeds by the flavourantiflavour pair firstly emitting a gluon with transverse-momentum choices as in the case of gluon decays discussed above, before then the heavier of the two colour dipoles, made of one of the two flavours and the gluon, decays further, exactly as above.

If in contrast a cluster falls into one or both transition regimes, probabilities for the correspondingly allowed transitions into one or two clusters are calculated. For the direct $C \rightarrow H$ transitions, this probability is given by

$$
\mathcal{P}_{M_{c} \rightarrow m_{h}}=\frac{\kappa}{2 M_{c}} \frac{\left(M_{c} \Gamma_{h}\right)^{2}}{\left(M_{c}^{2}-m_{h}^{2}\right)^{2}+\left(m_{h} \Gamma_{h}\right)^{2}}\left|\Psi_{q \bar{q}}(h)\right|^{2},
$$

where $m_{h}$ and $\Gamma_{h}$ respectively are the mass and width of the hadron in question, and where $\Psi_{q \bar{q}}(h)$ denotes the corresponding flavour wavefunction component of the hadron (e.g. $\Psi_{u \bar{u}}\left(\pi^{0}\right)=1 / \sqrt{2}$ ). For 
decays $\mathrm{C} \rightarrow \mathrm{H}_{1} \mathrm{H}_{2}$, the probablity reads

$$
\mathcal{P}_{M_{c} \rightarrow m_{1} m_{2}}=\frac{\sqrt{\left[M_{c}^{2}-\left(m_{1}+m_{2}\right)^{2}\right]\left[M_{c}^{2}-\left(m_{1}-m_{2}\right)^{2}\right]}}{16 \pi M_{c}^{3}}\left[\frac{4 m_{1} m_{2}}{M_{c}^{2}}\right]^{\alpha}\left|\Psi_{q \bar{q}^{\prime}}\left(h_{1}\right)\right|^{2}\left|\Psi_{q^{\prime} \bar{q}}\left(h_{2}\right)\right|^{2} \cdot \mathcal{P}_{q^{\prime}} .
$$

The parameter $\kappa$ can be used to change the relative rate of transitions and decays. In the decay weights, the exponent $\alpha$ steers the preference of the decays for heavier hadrons. $\mathcal{P}_{q}$ is the probability to create an additional $q$-flavour pair in the field of the decaying cluster. In each channel, the weights are further modified by including weights for the various hadron multiplets. In addition, there is a singlet suppression factor to reduce transition probabilities to singlet-octet mixed states in the meson sector.

Once a decay into two hadrons is chosen, the decay kinematics has to be fixed. This is achieved by giving the hadrons a transverse momentum w.r.t. the original flavour axis in the cluster rest frame. It is distributed according to

$$
p_{\perp}=|\vec{p}| \sin \theta \quad \text { where } \quad \sin \theta=\#^{\eta} .
$$

The parameter $\eta$ is adjustable and \# is a uniformly chosen random number between 0 and 1 .

\section{Decays of unstable particles}

Unstable particles can be produced in various phases of the event generation, for example in the signal process, or after fragmentation, or even from decays themselves.

For unstable particles generated by the hard process, SHERPA provides the possibility to specify the decay chain directly (cf. Sec. 3). Since the complete process is translated into Feynman diagrams, full correlations for all propagators are respected. This approach however is not ideal for particles with many decay channels as appearing for example in BSM models. In these cases one often wants to decay a particle from the signal process inclusively. Currently this is only possible for the $\tau$-leptons, which owing to their hadronic decay channels are treated by the hadron-decay module (cf. Sec. 8.1). A more general solution for other fundamental particles is being worked on for a future version of SHERPA.

On the other hand, unstable hadrons, generated e.g. during fragmentation, and the $\tau$-lepton can be inclusively decayed in cascade-like processes. Features of this implementation shall be described in the following.

A natural starting point for the description of such cascades is the branching ratios collected in the PDG tables [109], and to merely choose according to them the decay channel for individual hadrons. This is not always possible, since in many cases the respective branching ratios do not add up to one. There are several options to restore a complete decay table. For many of the light hadrons, especially in the cases of the pseudoscalar and vector-meson multiplets and the octet and decuplet baryons, it is viable to slightly rearrange the branching ratios, such that they add up to $100 \%$. Introducing new decay channels, "guesstimated" from flavour symmetries and phase-space arguments is an option for some of the higher, not well-measured resonances made of light quarks (like, e.g., the tensor mesons). But especially for hadrons involving heavy quarks, the sheer number of potentially open decay channels forbids such a treatment. Thus, a mixture of explicit hadron decays and transitions of the heavy-hadron state to quarks and gluons, invoking again the hadronisation model, turns out to be a much more powerful strategy. However, care has to be taken that the hadronic final state emerging after such a partonic decay has not already been covered by an explicit hadron-decay channel coexisting with the partonic mode. In the hadron-decay framework of SHERPA this is realised by explicitly vetoing all unwanted hadronic final states emerging after the hadronisation of a partonic decay.

Having selected a specific decay mode, its decay kinematics needs to be modelled. In order to go beyond an isotropic distribution of decay products in the phase space, explicit matrix elements are employed. They are usually inferred from the spin structure and similar symmetries of the initial and final state, giving rise to only a few amplitudes. This simple picture can be further refined by invoking form factors (FFs) for certain transitions - notable examples are the weak transitions of heavy quarks of the type $b \rightarrow c$ or $b \rightarrow u$, typically used in semileptonic decays of heavy hadrons (cf. Sec. 8.2.1), or FFs emerging for hadronic currents in $\tau$-decays (cf. Sec. 8.1).

The complexity of the emerging hadronic final-states and the multitude of hadron decays make it impossible to implement and calculate matrix elements and phase space for a full final state consisting of stable 
particles only. It is necessary to resort to the construction of "chains" of subsequent decays. Naively, it looks like a reasonable as well as feasible approximation to deal with each decay of such a chain completely independently. Nevertheless, looking a bit more carefully reveals that complete independence is not always a valid assumption. Clearly, the spin structure of decaying and intermediate particles potentially leads to non-trivial correlations among them and other intermediate particles possibly emerging in the primary decay. The algorithm presented in [110] has been implemented to take such non-trivial correlations into account. To illustrate it, consider the decay $B \rightarrow D \rho$ with a subsequent $\rho \rightarrow \pi \pi$ decay. Naively, neglecting form factors, the full matrix element for $B \rightarrow D \pi \pi$ can be described by

$$
\begin{aligned}
\mathcal{M} & \sim i\left(p_{B}^{\mu}+p_{D}^{\mu}\right) \cdot\left[g_{\mu \nu}-\frac{p_{\mu} p_{\nu}}{p^{2}}\right] \cdot\left(p_{\pi_{1}}^{\nu}-p_{\pi_{2}}^{\nu}\right) \\
& \rightarrow i \sum_{\lambda_{\rho}}\left(p_{B}^{\mu}+p_{D}^{\mu}\right) \cdot \varepsilon_{\mu}^{*}\left(\lambda_{\rho}\right) \varepsilon_{\nu}\left(\lambda_{\rho}\right) \cdot\left(p_{\pi_{1}}^{\nu}-p_{\pi_{2}}^{\nu}\right) .
\end{aligned}
$$

This then allows to write the matrix element for $B \rightarrow D \pi \pi$ in factorised form as

$$
\mathcal{M} \sim \sum_{\lambda_{\rho}} \mathcal{M}\left(B \rightarrow D \rho ; \lambda_{\rho}\right) \mathcal{M}\left(\rho \rightarrow \pi \pi ; \lambda_{\rho}\right)
$$

Here the only difference to the decay cascade is that the sum over helicities $\lambda_{\rho}$ is not computed independently for the two subprocesses. The spin-correlation algorithm takes this into account.

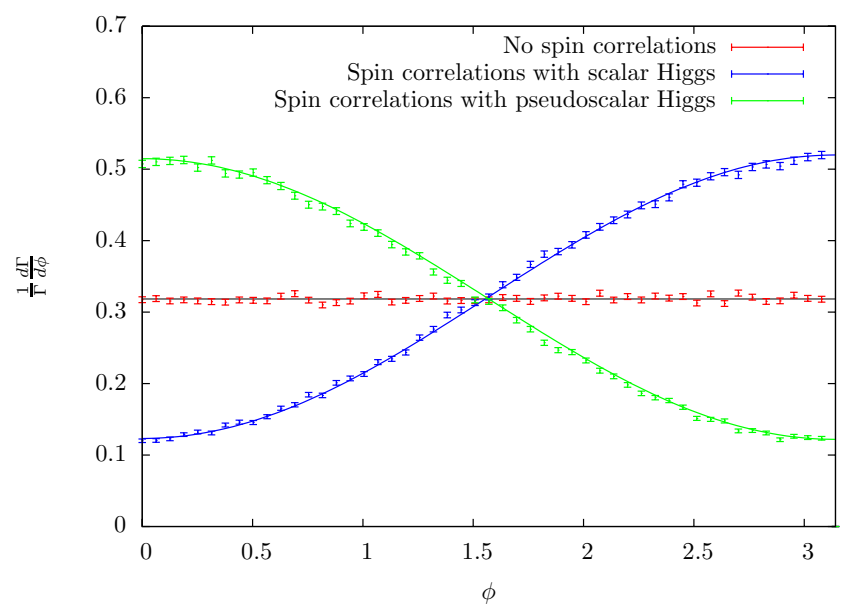

Fig. 17 Effect of spin correlations for the angle between the decay planes in $h \rightarrow \tau^{-} \tau^{+} \rightarrow \pi^{-} \nu_{\tau} \pi^{+} \bar{\nu}_{\tau}$

Spin correlations for $\tau$-leptons produced by the hard matrix element are also taken into account when decaying the $\tau$. An example for this can be seen in Fig. 17 displaying the angle between the decay planes of $\tau^{-}$and $\tau^{+}$produced from a Higgs boson decay. The solid lines represent the analytical predictions taken from [111].

Unstable particles such as the $\rho$ meson have a finite width, which may be quite large. In order to accomodate for this effect, the invariant mass of the decaying particle can a posteriori, i.e. after the particle has been produced on its mass shell, be smeared according to a relativistic Breit-Wigner distribution. When doing so, however, kinematic bounds must be respected. It is quite obvious that there is an upper bound given by the mass of the decaying particle subtracted by the masses of the other decay products, which themselves may have to be smeared. Hence, a strategy is needed concerning the sequence of the mass modifications. Apart from the upper limit, there is also a lower limit for the invariant mass of the decaying particle, given by the minimal mass of its decay products. This inevitably will vary with the decay channel - fixing the mass before fixing the decay channel could therefore lead to unwanted biases, modifying the branching ratios in an unacceptable way. In SHERPA, these two problems are solved by fixing the decay channels of the secondaries before applying the Breit-Wigner smearing on their masses, the latter with rising width of the particles.

This still leaves some room for improvement. Currently, the mass of any unstable particle is distributed according to a superposition of Breit-Wigner distributions with different weights given by the respective 
branching ratios and different lower cut-offs given by the minimal masses of the respective final state. This simple picture may be refined by adding in threshold effects emerging when new decay channels are becoming kinematically accessible. This can be done by reweigthing with the "true" invariant mass in the phase-space factors. For the dicing of the offshell mass $m_{a}^{\prime}$ of a particle $a$ decaying in a two-particle decay $a \rightarrow b c$, the emerging correction weight $w$ reads

$$
w_{\mathrm{PS}}=\frac{\mathrm{d} \Phi^{(2)}\left(m_{a}^{\prime 2}, m_{b}^{2}, m_{c}^{2}\right)}{\mathrm{d} \Phi^{(2)}\left(m_{a}^{2}, m_{b}^{2}, m_{c}^{2}\right)}=\frac{m_{a}^{3}}{m_{a}^{\prime 3}} \sqrt{\frac{\lambda\left(m_{a}^{\prime 2}, m_{b}^{2}, m_{c}^{2}\right)}{\lambda\left(m_{a}^{2}, m_{b}^{2}, m_{c}^{2}\right)}}
$$

with $\lambda(a, b, c)=(a-b-c)^{2}-4 b c$. For n-particle decays, one can resort to an approximation using the mass of the heaviest decay product for $m_{b}$ and the sum of the remaining decay-product masses for $m_{c}$. In SHERPA these phase-space correction weights for updated masses are used, but no correction for the matrix element is applied.

\section{1 $\tau$-lepton decays}

The $\tau$-lepton is the only lepton being heavy enough to decay into lighter hadrons. Consequently, it provides an excellent laboratory for measuring hadronic currents and, additionally, to test the Fermi point-like interaction assumption and the CVC (PCAC) hypothesis [112].

The mass of the $\tau$-lepton is roughly $1.77 \mathrm{GeV}$, which does not suffice to produce a charmed hadron while decaying. Therefore, its decay products are either leptons or light hadrons consisting merely of up, down, and strange quarks. For both, the matrix element of the decay

$$
\tau^{-}(P) \rightarrow \nu_{\tau}(k)+\text { products }
$$

is given by

$$
\mathcal{M}=\frac{G_{F}}{\sqrt{2}} \bar{u}\left(k, \lambda_{\nu_{\tau}}\right)\left(\gamma^{\mu}-\gamma^{\mu} \gamma_{5}\right) u\left(P, \lambda_{\tau}\right) J_{\mu}
$$

where the current $J_{\mu}$ depends on the chosen decay channel.

The branching ratio of the leptonic decay channels is about $35 \%$. This is a pure weak process, which can be calculated analytically and serves as a suitable testbed for the left-handed leptonic currents.

The more involved hadronic channels with more than one hadron have complicated resonance structures owing to intermediate particles with a short life time such as the $\rho$ meson. The corresponding decays are described by form factors, which in SHERPA can be parametrised according to the following models:

- Kühn-Santamaría(KS) [113] parametrisation,

- Resonance Chiral Theory $(\mathrm{R} \chi \mathrm{T})[114]$.

The KS model is a phenomenological approach describing resonances through their Breit-Wigner form. They are introduced "by hand" into the matrix element. $\mathrm{R} \chi \mathrm{T}$ on the other hand is an effective field theory: the extrapolation of Chiral Perturbation Theory $(\chi \mathrm{PT})$ to higher energies. Using this approach, the decay matrix elements can be calculated analytically including all occuring resonances.

Such form factors have been implemented for decays into up to three pseudoscalars of the type $\pi^{ \pm}, \pi^{0}$, $K^{ \pm}, K^{0}$ and the two modes producing four pions. As an example, Fig. 18 shows a comparison of four different form-factor models with CLEO data [115].

\subsection{Hadron decays}

With approximately 200 decay tables consisting of 2500 decay channels the majority of the decaying particles are hadrons. For some of them, form factors have been very accurately measured, while for others not even the branching ratios are well known. In the following the features of hadron-decay simulations in SHERPA will be described thereby focussing on heavy mesons. Details about the complete package can be found in [38]. 


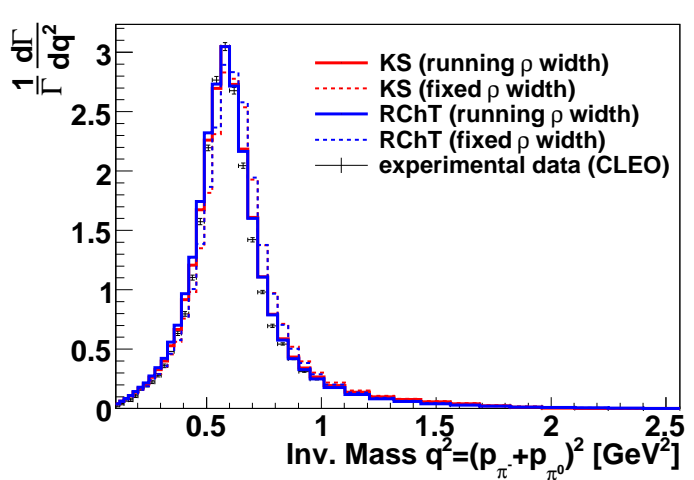

Fig. 18 Invariant mass distribution of the two outgoing pions for $\tau^{-} \rightarrow \nu_{\tau} \pi^{-} \pi^{0}$.

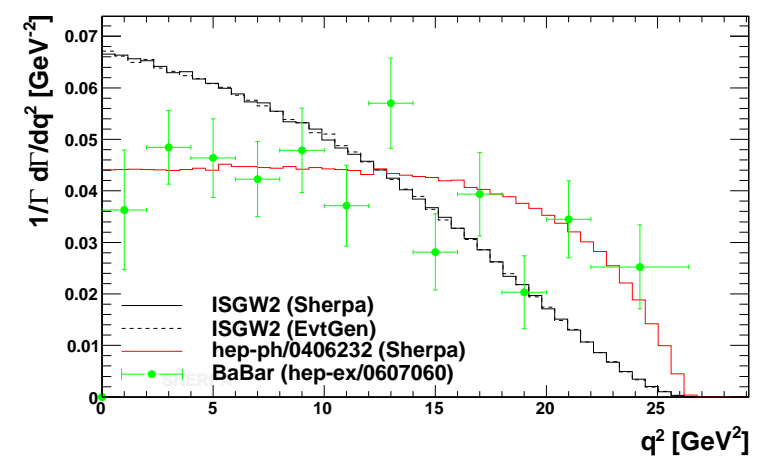

Fig. 19 Invariant mass of the lepton-neutrino pair for the decay $B \rightarrow \pi \nu_{e} e$.

\subsubsection{Heavy mesons}

Mesons containing one heavy quark $\left(m_{q} \gg \Lambda_{\mathrm{QCD}}\right)$ as a constituent can appear as

- $D^{+}(c \bar{d})$ and $D^{-}(\bar{c} d)$,

- $D^{0}(c \bar{u})$ and $\overline{D^{0}}(\bar{c} u)$,

- $D_{s}^{+}(c \bar{s})$ and $D_{s}^{-}(\bar{c} s)$,

- $B^{+}(\bar{b} u)$ and $B^{-}(b \bar{u})$,

- $B^{0}(\bar{b} d)$ and $\overline{B^{0}}(b \bar{d})$,

- $B_{s}^{0}(\bar{b} s)$ and $\overline{B_{s}^{0}}(b \bar{s})$,

- $B_{c}^{+}(\bar{b} c)$ and $B_{c}^{-}(b \bar{c})$.

In most weak decays, only the heavy quark undergoes the flavour change and enters into the $V-A$ current. The second quark only serves as "spectator" being exchangeable among the light quarks.

For mesons with heavy spectator quarks, additional decay modes may appear, because the spectator quark can decay weakly as well. Differences may also appear in the form factors, such that separate ones are implemented for decays of the $B_{c}$ meson.

Excited mesons, like the $B^{*}$, do not feature very diversified decay channels, but decay to their appropriate ground states emitting an additional pion or photon. No form factors have been implemented for these two-body decays, but they are treated according to the respective generic matrix element.

The main decay modes of the pseudoscalar ground states fall into three categories: leptonic decays, semileptonic decays and purely hadronic decays. Leptonic decay channels are easily parametrised using decay constants and generic matrix elements.

For semileptonic decays, just like for $\tau$ decays, it is useful to simply assume a factorised form of the decay matrix elements: in this case the heavy-to-heavy or heavy-to-light transitions can be modelled with form factors, which are determined by effective field-theory approaches such as Heavy Quark Effective Theory (HQET) [116], quark-model predictions [117] or QCD sum rules [118]. Fig. 19 exemplifies the use of different form factor models in the decay $B \rightarrow \pi \nu_{e} e$ by comparing to predictions by the EvtGen simulation package [119] and data from BABAR [120]. For hadronic decays, again a factorisation between the two hadronic currents is assumed, using the heavy currents from the semileptonic decays and combining them with a hadronic current taken over from $\tau$-decays. In fact, for a certain class of heavy-to-light transitions, this factorisation ansatz has been proven to yield results correct up to higher orders [121], and it will be used for these and many other decays in SHERPA. 


\subsubsection{Meson oscillations and CP violation}

In addition to spin correlations and finite-width effects, more improvements beyond the decay cascade are related to the simulation of mixing phenomena in systems of neutral mesons, most notably $B \bar{B}$-mixing, and the modelling of CP-violating effects.

In the following the relationship between mass eigenstates $M_{L / H}$ and flavour eigenstates $M^{0} / \overline{M^{0}}$ of a neutral meson $M$ is defined as

$$
\left|M_{L}\right\rangle=p\left|M^{0}\right\rangle+q\left|\overline{M^{0}}\right\rangle \quad \text { and } \quad\left|M_{H}\right\rangle=p\left|M^{0}\right\rangle-q\left|\overline{M^{0}}\right\rangle .
$$

This leads to a non-trivial time evolution $M_{\text {phys }}^{0}(t)$ for flavour eigenstates produced at $t=0$ in fragmentation and decay processes. Four related effects are implemented in SHERPA:

1. Explicit mixing in the event record can be accomplished by setting the parameters $\Delta m=m_{H}-m_{L}$ and $\Delta \Gamma=\Gamma_{H}-\Gamma_{L}$ of the neutral meson. According to the time evolution of the flavour eigenstates, the mesons will then decay as the appropriate flavour.

2. CP violation "in mixing" can be simulated by setting the value of $\left|\frac{q}{p}\right|^{2} \neq 1$.

3. Direct CP violation "in decays" can be accounted for by specifying different decay tables for particles and their antiparticles.

4. CP violation "in the interference of mixing and decays" appears in the decay to final states common to meson and anti-meson, because of interference terms between the amplitude with a mixed meson and the unmixed amplitude. It leads to a time dependent rate asymmetry

$$
A_{C P}(t)=\frac{\Gamma\left(\bar{M}_{\mathrm{phys}}^{0}(t) \rightarrow f\right)-\Gamma\left(M_{\mathrm{phys}}^{0}(t) \rightarrow f\right)}{\Gamma\left(\bar{M}_{\mathrm{phys}}^{0}(t) \rightarrow f\right)+\Gamma\left(M_{\mathrm{phys}}^{0}(t) \rightarrow f\right)}
$$

Defining the quantity $\lambda_{f}=\frac{q}{p} \frac{\overline{\mathcal{M}}_{f}}{\mathcal{M}_{f}}$ for a decay to the common final state $f$ through the matrix elements $\mathcal{M}$, the asymmetry can be expressed as

$$
A_{C P}(t)=\frac{S \cdot \sin (\Delta m t)-C \cdot \cos (\Delta m t)}{\cosh \left(\Delta \Gamma \frac{t}{2}\right)-\frac{2 \operatorname{Re} \lambda_{f}}{1+\left|\lambda_{f}\right|^{2}} \sinh \left(\Delta \Gamma \frac{t}{2}\right)} \stackrel{\Delta \Gamma=0}{=} S \cdot \sin (\Delta m t)-C \cdot \cos (\Delta m t)
$$

where $S=\frac{2 \operatorname{Im} \lambda_{f}}{1+\left|\lambda_{f}\right|^{2}}$ and $C=\frac{1-\left|\lambda_{f}\right|^{2}}{1+\left|\lambda_{f}\right|^{2}}$ denote the mixing-induced and direct contributions to the CP asymmetry, respectively.

The factors $S$ and $C$ have been measured or calculated for many decay channels of the $B$ meson, cf. [109] for a listing of measured values. If $\Delta \Gamma \neq 0$ is chosen, SHERPA calculates $\lambda_{f}$ from the given values of $S$ and $C$,

$$
\begin{aligned}
\left|\lambda_{f}\right|^{2} & =\frac{1-C}{1+C}, \\
\operatorname{Im}\left(\lambda_{f}\right) & =\frac{S}{1+C}, \\
-\frac{2 \operatorname{Re} \lambda_{f}}{1+\left|\lambda_{f}\right|^{2}} & =-\sqrt{1-C^{2}-S^{2}},
\end{aligned}
$$

and simulates according to the non-simplified expression for $A_{C P}(t)$ given in Eq. (43).

A simulation of CP violation owing to interference effects in the decays of $B^{0} \bar{B}^{0}$ pairs created from $\Upsilon(4 S)$ decays is presented in Fig. 20. Here, one of the $B$ mesons is randomly selected to decay semileptonically at time $t_{0}$. This provides a flavour tag at $t_{0}$ for the other $B$, decaying at $t_{1}$ into $J / \Psi K_{S}$. Since both $B$ mesons have been produced coherently in the $\Upsilon(4 S)$ decay, the reference time for the evolution of both flavour eigenstates is set by the semileptonic decay. Thus the rate asymmetry is governed by the time difference $\Delta t=t_{1}-t_{0}$, which can be positive or negative. 


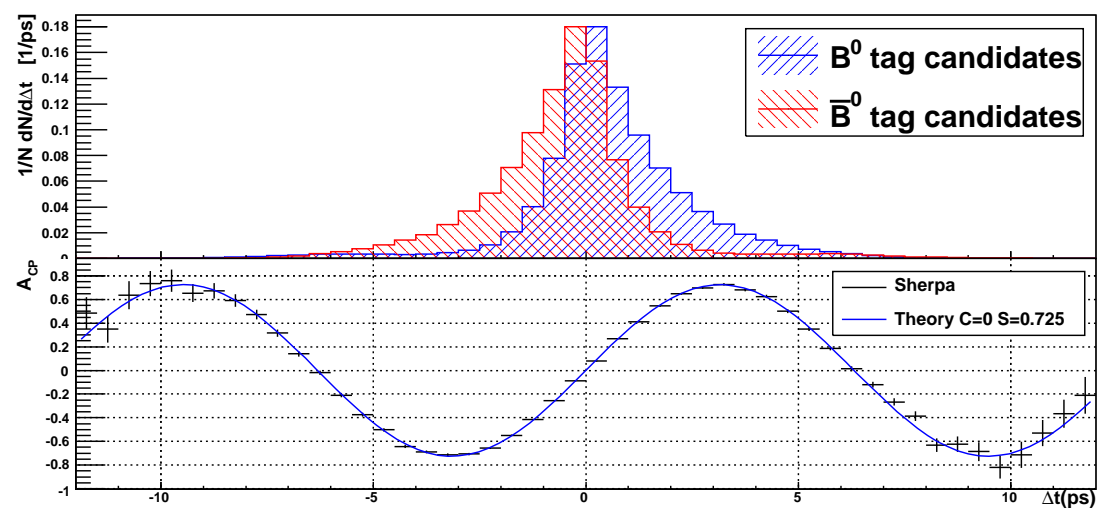

Fig. 20 CP violation in the interference between $B^{0} \rightarrow J / \Psi K_{S}$ and $\bar{B}^{0} \rightarrow J / \Psi K_{S}$ with $\Delta \Gamma=0, S=0.725$ and $C=0$.

\section{Multiple emission of photons}

In the previous sections nearly all possible aspects of event generation were adressed. However, higher order QED corrections in the form of soft-photon radiation were neglected. Nonetheless, soft-photon radiation occurs at any stage of event generation whenever charged particles are involved. They affect, e.g., production cross sections, (partial) decay widths of hadrons and $\tau$-leptons, and broaden momentum and invariant-mass distributions of final-state particles. Therefore these corrections have to be taken into account in a realistic simulation. In SHERPA this is accomplished by the PHOTONS++ module, which is based on the approach of Yennie, Frautschi and Suura (YFS) [40].

The corresponding algorithm relies on the idea of resumming leading soft logarithms to all orders, rather than focusing on leading collinear terms, which are taken into account in conventional parton showers (cf. Sec. 4). Soft logarithms are largely independent of the inner process characteristics and can be calculated from the charges of the external particles and their four-momenta only. The YFS formalism allows a systematic improvement of the eikonal approximation order-by-order in the QED coupling constant - which has rendered it the standard approach for precision-calculations of QED radiation effects [122].

At present the Photons++ module is only capable to handle single-particle initial states and, hence, can only effect these corrections for particle decays. For the hard process, collinearly enhanced photon emission off charged leptons and quarks is accounted for during the parton-shower evolution, cf. Sec. 4.

\subsection{The formalism}

The YFS formalism shall be reviewed very briefly to illustrate the mechanisms of the resummed softphoton contributions and the order-by-order improvement for the hard emission region.

Consider a process at zeroth order in the electromagnetic coupling $\alpha$, described by the matrix element $\mathcal{M}_{0}^{0}$. The sub- and superscripts denote the number of real photons involved and the order of $\alpha$, respectively. The four-momenta of the final-state particles are labelled $p_{f}$, while the incoming momenta are denoted by $p_{i}$. The fully inclusive cross section for having any number of additional soft photons with momenta $k$ can then be written as

$$
\sigma \sim \sum_{n_{R}=0}^{\infty} \frac{1}{n_{R} !} \int \mathrm{d} \Phi_{p} \mathrm{~d} \Phi_{k}(2 \pi)^{4} \delta^{4}\left(\sum p_{i}-\sum p_{f}-\sum k\right)\left|\sum_{n_{V}=0}^{\infty} \mathcal{M}_{n_{R}}^{n_{V}+\frac{1}{2} n_{R}}\right|^{2}
$$

where $n_{V}$ and $n_{R}$ respectively count the numbers of virtual and real photons involved.

The starting point of the YFS algorithm is to approximate the dressed matrix elements by the zerothorder expression times eikonal factors that depend on the external momenta only. The correct result can then be restored order-by-order in perturbation theory by supplementing the non-leading, processdependent pieces. In the case of adding just one virtual photon this can be formalised as

$$
\mathcal{M}_{0}^{1}=\alpha B \mathcal{M}_{0}^{0}+M_{0}^{1},
$$


with $M_{0}^{1}$ being the infrared-subtracted matrix element including one extra virtual photon. Note that accordingly $M_{0}^{1}$ is finite in the limit of vanishing photon momentum, i.e. $k \rightarrow 0$. All soft divergences owing to the virtual-photon insertion are contained in the integrated eikonal $B$, which is process-independent and universal ${ }^{6}$. When summing over all subsequent insertions of further virtual photons, thereby iteratively applying Eq. (48), the very appealing all-order expression

$$
\sum_{n_{V}=0}^{\infty} \mathcal{M}_{0}^{n_{V}}=\sum_{n_{V}=0}^{\infty} \sum_{r=0}^{n_{V}} M_{0}^{n_{V}-r} \frac{(\alpha B)^{r}}{r !}=\exp (\alpha B) \sum_{n_{V}=0}^{\infty} M_{0}^{n_{V}}
$$

can be derived. This can be generalised to any number of real photons by exploiting the unique characteristics of abelian QED and the fact that virtual photons inserted in closed charged loops do not produce any additional infrared singularity. Hence, all $M_{n_{R}}^{n_{V}+\frac{1}{2} n_{R}}$ are free of soft singularities from virtual-photon exchange but may still exhibit such due to real-photon emission.

However, YFS were able to show that real-photon emission processes can also be factorised - on the level of the squared matrix elements rather than the amplitudes. For a single radiated photon, one obtains

$$
\frac{1}{2(2 \pi)^{3}}\left|\sum_{n_{V}=0}^{\infty} M_{1}^{n_{V}+\frac{1}{2}}\right|^{2}=\tilde{S}(k)\left|\sum_{n_{V}=0}^{\infty} M_{0}^{n_{V}}\right|^{2}+\sum_{n_{V}=0}^{\infty} \tilde{\beta}_{1}^{n_{V}+1}(k) .
$$

Here, $\tilde{S}(k)$ is an eikonal factor containing the soft divergence related to real-photon emission ${ }^{7} . \tilde{\beta}_{n_{R}}^{n_{V}}+n_{R}$ denotes the complete IR-finite (subtracted) squared matrix element for the basic process dressed with $n_{R}$ real and $n_{V}$ virtual photons. With Eq. (51) at hand the perturbative series can be reordered such that a complete partition of infrared finite and divergent terms is achieved. Moreover, the singular terms can be exponentiated by separating the real-emission phase space into a region $\Omega$ containing the infrared divergence such that $(1-\Omega)$ is completely IR finite. Introducing the YFS form factor ${ }^{8}$

$$
Y(\Omega)=2 \alpha(B+\tilde{B}(\Omega))
$$

the cancellation of real and virtual infrared divergences can be made explicit. The rearranged form of the exact cross section given in Eq. (47) reads

$$
\begin{array}{r}
\sigma \sim \sum_{n_{R}=0}^{\infty} \frac{1}{n_{R} !} \int \mathrm{d} \Phi_{p} \mathrm{~d} \Phi_{k}^{\prime}(2 \pi)^{4} \delta^{4}\left(\sum p_{i}-\sum p_{f}-\sum k\right) e^{Y(\Omega)} \prod_{i=1}^{n_{R}} \tilde{S}\left(k_{i}\right) \Theta\left(k_{i}, \Omega\right) \\
\times\left(\tilde{\beta}_{0}^{0}+\tilde{\beta}_{0}^{1}+\sum_{i=1}^{n_{R}} \frac{\tilde{\beta}_{1}^{1}\left(k_{i}\right)}{\tilde{S}\left(k_{i}\right)}+\mathcal{O}\left(\alpha^{2}\right)\right) .
\end{array}
$$

The original perturbative series, organised in powers of the electromagnetic coupling constant $e$ and based on amplitudes, has been rearranged as a perturbative series in $\alpha$ based on squared matrix elements, whose infrared singularities have been extracted. While the squared matrix elements encode all the processspecific information, e.g. spin correlations, interferences, and hard-emission properties, the YFS form factor describes the resummed universal soft limit.

$$
\begin{aligned}
& { }^{6} \text { The universal integrated virtual eikonal is a sum over the eikonals of every QED subdipole, i.e. } \\
& B=\sum_{i<j} B_{i j} .
\end{aligned}
$$

Details can be found in [39].

7 The universal real eikonal is defined as a sum over subdipoles

$$
\tilde{S}(k)=\sum_{i<j} \tilde{S}_{i j}(k) \text {. }
$$

For details see [39].

8 The integrated real-emission eikonal - in analogy to the universal virtual eikonal $B$ - is defined as

$$
2 \alpha \tilde{B}(\Omega)=\int \frac{\mathrm{d}^{3} k}{k} \tilde{S}(k)(1-\Theta(k, \Omega)),
$$

with $\Theta(k, \Omega)=1$ if $k \notin \Omega$ and zero otherwise. 
In this apparent form of the all-orders cross section the leading-order process $\tilde{\beta}_{0}^{0}$ can be factored out enabling the construction of a Monte Carlo algorithm to correct (arbitrary) cross sections or decay rates for real and virtual QED radiation.

\subsection{The algorithm}

In Ref. [39] a concrete version of such an algorithm is presented. Two basic aspects of this ansatz shall briefly be discussed here, the definition of the soft region $\Omega$ and the reconstruction algorithm for the leading-order particles' momenta.

To simplify calculations the singular phase-space region $\Omega$ has been chosen to be bounded by an isotropic hypersurface in the rest frame of the multipole, i.e. the ensemble of charged particles involved in the considered process. It is specified by an energy cut-off $\omega$. Photons outside $\Omega$ are generated exclusively. Their number, energy and angular distribution are determined by $\int \frac{\mathrm{d}^{3} k}{k} \tilde{S}(k) \Theta(k, \Omega)$, the soft real-emission eikonal. The higher-order hard-emission corrections are then accomodated by introducing them as a relative weight.

This can be achieved by approximating the real-emission matrix elements in the quasi-collinear limit using the QED variant of the spin-dependent dipole splitting functions $g_{i j}$ of [123]. However, the terms describing the soft limit need to be subtracted as they are already included in the YFS form factor. This yields new, soft-subtracted, dipole splitting functions denoted by $\bar{g}_{i j}$. For the collinearly approximated hard-emission matrix elements one then obtains

$$
\tilde{\beta}_{1}^{1}=-\frac{\alpha}{4 \pi^{2}} \sum_{i<j} Z_{i} Z_{j} \theta_{i} \theta_{j}\left(\bar{g}_{i j}+\bar{g}_{j i}\right) \tilde{\beta}_{0}^{0} .
$$

However, the $\tilde{\beta}_{1}^{1}$ lack any interference terms between the different amplitudes entering. These can only be incorporated using the exact real-emission matrix element. This, however, is process specific and, hence, is only available for some special cases (a list of available matrix elements is given in Tab. 3). Furthermore, to maintain a process-independent formulation as far as possible, extended, composite objects, e.g. hadrons, need to be treated as point-like ones. To achieve a higher accuracy in selected channels form-factor models can be used.

\begin{tabular}{|c|c|c|}
\hline matrix element & real $\mathcal{O}\left(\alpha_{\mathrm{QED}}\right)$ & virtual $\mathcal{O}\left(\alpha_{\mathrm{QED}}\right)$ \\
\hline$V^{0} \rightarrow F^{+} F^{-}$ & $\sqrt{ }$ & $\sqrt{ }$ \\
$V^{0} \rightarrow S^{+} S^{-}$ & $\sqrt{ }$ & $\sqrt{ }$ \\
$S^{0} \rightarrow F^{+} F^{-}$ & $\sqrt{ }$ & $\sqrt{ }$ \\
$S^{0} \rightarrow S^{+} S^{-}$ & $\sqrt{ }$ & $\sqrt{ }$ \\
$W^{ \pm} \rightarrow \ell^{ \pm} \nu_{\ell}$ & $\sqrt{ }$ & - \\
$\tau^{ \pm} \rightarrow \ell^{ \pm} \nu_{\ell} \nu_{\tau}$ & $\sqrt{ }$ & - \\
$S^{0} \rightarrow S^{\mp} \ell^{ \pm} \nu_{\ell}$ & under construction \\
$S^{0} \rightarrow V^{\mp} \ell^{ \pm} \nu_{\ell}$ & under construction & - \\
\hline
\end{tabular}

Tab. 3 List of available generic and specific infrared subtracted squared real-emission $\left(\tilde{\beta}_{1}^{1}\right)$ and virtualcorrection $\left(\tilde{\beta}_{0}^{1}\right)$ matrix elements $(V$ - vector, $F$ spin- $\frac{1}{2}$ fermion, $S$ - scalar).

A comparison of the impact of the various possibilities to incorporate hard-emission effects can be found in Fig. 21, which depicts the total amount of energy radiated and the angular radiation spectrum in leptonic $J / \psi$-decays, respectively. Both distributions highlight the importance of properly accounting for hard radiation and reveal the shortcomings of the approximated matrix elements when compared to the exact result. The approximation overestimates hard radiation at large angles, thus enhances the total fraction of events with hard photons close to the kinematic limit at half the mass of the decaying particle. This effect originates from the missing interference terms and is the more pronounced the higher the mass of the emitting particle. The soft limit of course is the same in all approaches, since it is incorporated in the YFS form factor $Y(\Omega)$ to all orders in $\alpha$.

The original particles' momenta need to be adapted to satisfy momentum conservation after the higherorder QED corrections have been included. This is achieved by distributing the additional photons' 

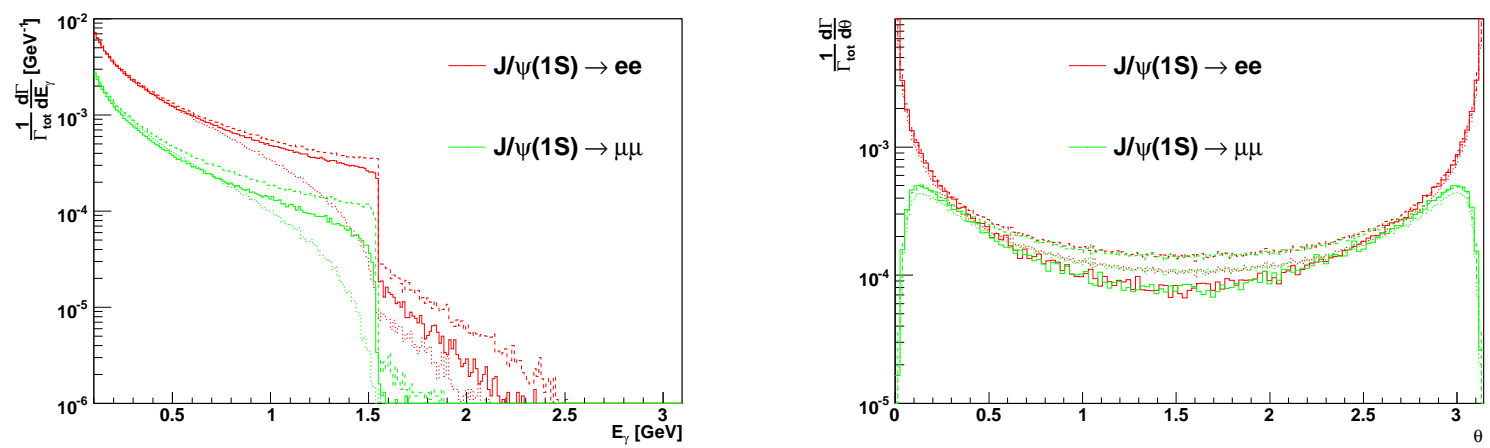

Fig. 21 Left panel: Total photon energy radiated for $J / \psi(1 S) \rightarrow \ell^{+} \ell^{-}$in the $J / \psi$ rest frame. Right panel: Angular distribution of photon radiation for $J / \psi(1 S) \rightarrow \ell^{+} \ell^{-}$in the $\ell^{+}-\ell^{-}$rest frame. The effects of different types of higher-order hard-emission corrections are shown: no corrections (dotted), approximated real-emission matrix-element correction (dashed) and exact real-emission matrix-element correction (solid).

momenta uniformly among all, charged and neutral, particles of the process, supplemented by a common rescaling of all final-state three-momenta in the multipole's rest frame. This prescription necessitates a change of the initial-state momenta as well. However, since these were already fixed when calculating the leading-order process, this change is interpreted as a shift of the multipole's rest frame. Additional complications arise for initial states involving more than one particle, however, at present PHOTONS++ is limited to particle decays.

On the other hand, photons emitted inside $\Omega$ are treated inclusively and although their number is infinite, the sum of their momenta is assumed to have a negligible effect on the overall momentum distribution.

\section{Summary}

In this article the original publication on the proof-of concept version of SHERPA [5], dating back to 2003, has been updated. Since then, SHERPA has matured considerably, especially in terms of physics capabilities, reliability and user support. At the same time, however, the original strategy of a separation of physics modules and structures for event generation has been maintained. In fact, the underlying idea of the physics being confined to individual modules, steered by corresponding handler classes, which in turn are employed by appropriate event phase classes during event generation, has proven to be extremely flexible, robust and versatile. Eventually, this construction paradigm has allowed a comparably quick and painless incorporation of many new physics aspects and additional user interfaces. This has helped to augment SHERPA with many hitherto missing physics aspects, for example hadronisation, hadron decays and the radiation of soft photons, thus replacing interfaces to already existing Fortran routines from other codes $^{9}$. These additions render the program a truly self-contained event generator for lepton-lepton and hadron-hadron collisions. In addition, many existing physics modules have been upgraded or improved. This ranges from the implementation of some models for new physics over a significantly enhanced and validated merging prescription for matrix elements and parton showers to the incorporation of a number of interfaces to, e.g., the Les Houches accord files for transmitting MSSM parameters or the LHAPDF library of PDFs. In addition, the user-interface has significantly improved, now including, among others, various event formats, a large variety of phase-space cuts and the greatly enhanced SHERPA-internal analysis tool. It is worth stressing that all these improvements certainly enhance both the reliability and the physics abilities of SHERPA.

In addition to the published features of SHERPA described in this article, many other features have established, but are not yet part of a publicly available version. These features include two new QCD shower modules, one based on Catani-Seymour dipole factorisation [124] and a dipole shower [125]. In the matrix-element sector, an automated Catani-Seymour dipole subtraction algorithm has been

\footnotetext{
${ }^{9}$ For the sake of backward-compatibility, these interfaces are kept available.
} 
implemented within AMEGIC++ [126], and a new matrix-element generator for very large multiplicities, COMIX [57], has been constructed.

These new modules, have not been released yet as part of SHERPA, but are planned to be included in a version 1.2, which will be made available in 2009. Their incorporation can be seen as a first step of the package being upgraded to next-to-leading order accuracy in the matrix-element sector. In this context, the initial construction paradigm of SHERPA again proves to yield a very flexible code, which can easily support the newly emerging structures.

\section{Acknowledgements}

T.G.'s research was supported by the US Department of Energy, contract DE-AC02-76SF00515. S.H. acknowledges funding by the HEPTOOLS Marie Curie Research Training Network (contract number MRTN-CT-2006-035505) and the Swiss National Science Foundation (SNF, contract number 200020117602). F.S.'s research was funded by MCnet (contract number MRTN-CT-2006-035606). S.S. acknowledges funding by the UK Sience and Technology Facilities Council (STFC). J.W. thanks O. González for helpful discussions. Fermilab is operated by Fermi Research Alliance, LLC, under contract DE-AC0207CH11359 with the United States Department of Energy. Financial support from BMBF is gratefully acknowledged.

\section{References}

[1] T. Sjöstrand, S. Mrenna and P. Skands, PYTHIA 6.4 physics and manual, JHEP 05 (2006), 026, [hep-ph/0603175].

[2] G. Corcella et al., HERWIG 6.5 Release Note, hep-ph/0210213.

[3] T. Sjöstrand, S. Mrenna and P. Skands, A brief introduction to PYTHIA 8.1, Comput. Phys. Commun. 178 (2008), 852-867, [arXiv:0710.3820 [hep-ph]].

[4] M. Bähr et al., Herwig++ Physics and Manual, arXiv:0803.0883 [hep-ph].

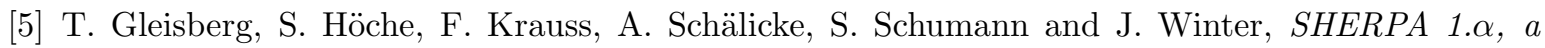
proof-of-concept version, JHEP 02 (2004), 056, [hep-ph/0311263].

[6] J. Campbell and R. K. Ellis, MCFM - Monte Carlo for FeMtobarn processes, http://mcfm.fnal. gov.

[7] Z. Nagy, Next-to-leading order calculation of three-jet observables in hadron-hadron collisions, Phys. Rev. D68 (2003), 094002, [hep-ph/0307268].

[8] S. Frixione and B. R. Webber, Matching NLO QCD computations and parton shower simulations, JHEP 06 (2002), 029, [hep-ph/0204244]. S. Frixione, P. Nason and B. R. Webber, Matching NLO QCD and parton showers in heavy flavour production, JHEP 08 (2003), 007, [hep-ph/0305252].

[9] S. Frixione, P. Nason and C. Oleari, Matching NLO QCD computations with parton shower simulations: the POWHEG method, JHEP 11 (2007), 070, [arXiv:0709.2092 [hep-ph]].

[10] S. Frixione and B. R. Webber, The MC@NLO 3.3 Event Generator, hep-ph/0612272.

[11] O. Latunde-Dada, S. Gieseke and B. Webber, A positive-weight next-to-leading-order Monte Carlo for $e^{+} e^{-}$annihilation to hadrons, JHEP 02 (2007), 051, [hep-ph/0612281]. K. Hamilton, P. Richardson and J. Tully, A Positive-Weight Next-to-Leading Order Monte Carlo Simulation of Drell-Yan Vector Boson Production, arXiv:0806.0290 [hep-ph]. O. Latunde-Dada, Applying the POWHEG method to top pair production and decays at the ILC, arXiv:0806.4560 [hep-ph].

[12] S. Frixione, P. Nason and G. Ridolfi, The POWHEG-hvq manual version 1.0, arXiv:0707.3081 [hep-ph]. S. Alioli, P. Nason, C. Oleari and E. Re, NLO vector-boson production matched with shower in POWHEG, JHEP 07 (2008), 060, [arXiv:0805.4802 [hep-ph]]. 
[13] M. L. Mangano, M. Moretti, F. Piccinini, R. Pittau and A. D. Polosa, ALPGEN, a generator for hard multiparton processes in hadronic collisions, JHEP 07 (2003), 001, [hep-ph/0206293].

[14] E. Boos et al., CompHEP collaboration, CompHEP 4.4 - automatic computations from Lagrangians to events, Nucl. Instrum. Meth. A534 (2004), 250-259, [hep-ph/0403113]. A. Pukhov, CalcHEP 3.2: MSSM, structure functions, event generation, batchs, and generation of matrix elements for other packages, hep-ph/0412191.

[15] A. Kanaki and C. G. Papadopoulos, HELAC: A package to compute electroweak helicity amplitudes, Comput. Phys. Commun. 132 (2000), 306-315, [hep-ph/0002082]. C. G. Papadopoulos, PHEGAS: A phase-space generator for automatic cross-section computation, Comput. Phys. Commun. 137 (2001), 247-254, [hep-ph/0007335]. A. Cafarella, C. G. Papadopoulos and M. Worek, HELACPHEGAS: a generator for all parton level processes, arXiv:0710.2427 [hep-ph].

[16] T. Stelzer and W. F. Long, Automatic generation of tree level helicity amplitudes, Comput. Phys. Commun. 81 (1994), 357-371, [hep-ph/9401258]. J. Alwall et al., MadGraph/MadEvent v4: The new web generation, JHEP 09 (2007), 028, [arXiv:0706.2334 [hep-ph]].

[17] W. Kilian, T. Ohl and J. Reuter, WHIZARD: Simulating Multi-Particle Processes at LHC and ILC, arXiv:0708.4233 [hep-ph].

[18] F. Krauss, R. Kuhn and G. Soff, AMEGIC++ 1.0: A Matrix Element Generator In $C++$, JHEP 02 (2002), 044, [hep-ph/0109036].

[19] S. Catani, F. Krauss, R. Kuhn and B. R. Webber, QCD matrix elements + parton showers, JHEP 111 (2001), 063, [hep-ph/0109231].

[20] F. Krauss, Matrix elements and parton showers in hadronic interactions, JHEP 0208 (2002), 015, [hep-ph/0205283].

[21] L. Lönnblad, Correcting the colour-dipole cascade model with fixed order matrix elements, JHEP 05 (2002), 046, [hep-ph/0112284]. N. Lavesson and L. Lönnblad, $W+$ jets matrix elements and the dipole cascade, JHEP 07 (2005), 054, [hep-ph/0503293].

[22] M. L. Mangano, M. Moretti and R. Pittau, Multijet matrix elements and shower evolution in hadronic collisions: $W b \bar{b}+n$-jets as a case study, Nucl. Phys. B632 (2002), 343-362, [hep-ph/0108069]. M. L. Mangano, M. Moretti, F. Piccinini and M. Treccani, Matching matrix elements and shower evolution for top-pair production in hadronic collisions, JHEP 01 (2007), 013, [hep-ph/0611129].

[23] S. Höche et al., Matching Parton Showers and Matrix Elements, hep-ph/0602031.

[24] J. Alwall et al., Comparative study of various algorithms for the merging of parton showers and matrix elements in hadronic collisions, Eur. Phys. J. C53 (2008), 473-500, [arXiv:0706.2569 [hep-ph]].

[25] R. Kleiss and W. J. Stirling, Spinor techniques for calculating $p \bar{p} \rightarrow W^{ \pm} / Z^{0}+j e t s$, Nucl. Phys. B262 (1985), 235-262. A. Ballestrero, E. Maina and S. Moretti, Heavy quarks and leptons at $e^{+} e^{-}$ colliders, Nucl. Phys. B415 (1994), 265-292, [hep-ph/9212246].

[26] T. Gleisberg, F. Krauss, C. G. Papadopoulos, A. Schälicke and S. Schumann, Cross sections for multi-particle final states at a linear collider, Eur. Phys. J. C34 (2004), 173-180, [hep-ph/0311273].

[27] K. Hagiwara et al., Supersymmetry simulations with off-shell effects for the CERN LHC and an ILC, Phys. Rev. D73 (2006), 055005, [hep-ph/0512260].

[28] R. Kleiss and R. Pittau, Weight optimization in multichannel Monte Carlo, Comput. Phys. Commun. 83 (1994), 141-146, [hep-ph/9405257]. F. A. Berends, R. Pittau and R. Kleiss, All electroweak four-fermion processes in electron-positron collisions, Nucl. Phys. B424 (1994), 308, [hep-ph/9404313]. 
[29] G. P. Lepage, VEGAS - An Adaptive Multi-dimensional Integration Program, CLNS-80/447, http://www.slac. stanford. edu/spires/find/hep/www?r=CLNS-80/447.

[30] R. Kleiss, W. J. Stirling and S. D. Ellis, A new Monte Carlo treatment of multiparticle phase space at high energies, Comput. Phys. Commun. 40 (1986), 359.

[31] A. van Hameren and C. G. Papadopoulos, A hierarchical phase space generator for QCD antenna structures, Eur. Phys. J. C25 (2002), 563-574, [hep-ph/0204055].

[32] R. Kuhn, F. Krauss, B. Ivanyi and G. Soff, APACIC++ 1.0: A PArton Cascade In C++, Comput. Phys. Commun. 134 (2001), 223-266, [hep-ph/0004270].

[33] F. Krauss, A. Schälicke and G. Soff, APACIC++ 2.0: A PArton Cascade In C++, Comput. Phys. Commun. 174 (2006), 876-902, [hep-ph/0503087].

[34] S. Alekhin et al., HERA and the LHC - A workshop on the implications of HERA for LHC physics: Proceedings Part A, hep-ph/0601012.

[35] T. Sjöstrand and M. van Zijl, A multiple-interaction model for the event structure in hadron collisions, Phys. Rev. D36 (1987), 2019.

[36] T. D. Gottschalk, A realistic model for $e^{+} e^{-}$annihilation including parton bremsstrahlung effects, Nucl. Phys. B214 (1983), 201. T. D. Gottschalk, An improved description of hadronization in the QCD cluster model for $e^{+} e^{-}$annihilation, Nucl. Phys. B239 (1984), 349. B. R. Webber, A QCD model for jet fragmentation including soft gluon interference, Nucl. Phys. B238 (1984), 492. T. D. Gottschalk and D. A. Morris, A new model for hadronization and $e^{+} e^{-}$annihilation, Nucl. Phys. B288 (1987), 729.

[37] J.-C. Winter, F. Krauss and G. Soff, A modified cluster-hadronisation model, Eur. Phys. J. C36 (2004), 381-395, [hep-ph/0311085].

[38] F. Krauss, T. Laubrich and F. Siegert, Simulation of hadron decays in SHERPA, in preparation.

[39] M. Schönherr and F. Krauss, Soft Photon Radiation in Particle Decays in SHERPA, arXiv:0810.5071 [hep-ph].

[40] D. R. Yennie, S. C. Frautschi and H. Suura, The Infrared Divergence Phenomena and High-Energy Processes, Ann. Phys. 13 (1961), 379-452.

[41] E. Barberio and Z. Wạs, PHOTOS - a universal monte carlo for QED radiative corrections: version 2.0, Comput. Phys. Commun. 79 (1994), 291-308.

[42] M. R. Whalley, D. Bourilkov and R. C. Group, The Les Houches Accord PDFs (LHAPDF) and LHAGLUE, hep-ph/0508110.

[43] P. Skands et al., SUSY Les Houches accord: interfacing SUSY spectrum calculators, decay packages, and event generators, JHEP 07 (2004), 036, [hep-ph/0311123].

[44] B. Badelek et al., ECFA/DESY Photon Collider Working Group collaboration, TESLA Technical Design Report, Part VI, Chapter 1: Photon collider at TESLA, Int. J. Mod. Phys. A19 (2004), 5097-5186, [hep-ex/0108012].

[45] A. F. Żarnecki, CompAZ: Parametrization of the luminosity spectra for the photon collider, Acta Phys. Polon. B34 (2003), 2741-2758, [hep-ex/0207021].

[46] V. M. Budnev, I. F. Ginzburg, G. V. Meledin and V. G. Serbo, The two photon particle production mechanism. Physical problems. Applications. Equivalent photon approximation, Phys. Rept. 15 (1974), 181-281.

[47] J. Archibald, T. Gleisberg, S. Höche, F. Krauss, M. Schönherr, S. Schumann, F. Siegert and J.-C. Winter, Simulation of photon-photon interactions in hadron collisions with SHERPA, Nucl. Phys. 179 (2008), 218-225. 
[48] M. Glück, E. Reya and A. Vogt, Parton structure of the photon beyond the leading order, Phys. Rev. D45 (1992), 3986-3994. M. Glück, E. Reya and A. Vogt, Photonic parton distributions, Phys. Rev. D46 (1992), 1973-1979.

[49] T. Appelquist and C. W. Bernard, Strongly interacting Higgs bosons, Phys. Rev. D22 (1980), 200. T. Appelquist and G.-H. Wu, Electroweak chiral Lagrangian and new precision measurements, Phys. Rev. D48 (1993), 3235-3241, [hep-ph/9304240].

[50] A. Dedes, T. Figy, S. Hoche, F. Krauss and T. E. J. Underwood, Searching for Nambu-Goldstone Bosons at the LHC, arXiv:0807.4666 [hep-ph].

[51] J. C. Pati and A. Salam, Mirror Fermions, J/psi Particles, Kolar Mine Events and Neutrino Anomaly, Phys. Lett. B58 (1975), 333-337. L. J. Hall and A. E. Nelson, Heavy Gluons and Monojets, Phys. Lett. B153 (1985), 430. P. H. Frampton and S. L. Glashow, Chiral Color: An Alternative to the Standard Model, Phys. Lett. B190 (1987), 157. P. H. Frampton and S. L. Glashow, Unifiable Chiral Color With Natural GIM Mechanism, Phys. Rev. Lett. 58 (1987), 2168. J. Bagger, C. Schmidt and S. King, Axigluon Production in Hadronic Collisions, Phys. Rev. D37 (1988), 1188 .

[52] N. Arkani-Hamed, S. Dimopoulos and G. R. Dvali, The hierarchy problem and new dimensions at a millimeter, Phys. Lett. B429 (1998), 263-272, [hep-ph/9803315]. I. Antoniadis, N. Arkani-Hamed, S. Dimopoulos and G. R. Dvali, New dimensions at a millimeter to a fermi and superstrings at a TeV, Phys. Lett. B436 (1998), 257-263, [hep-ph/9804398].

[53] T. Gleisberg, F. Krauss, K. T. Matchev, A. Schälicke, S. Schumann and G. Soff, Helicity formalism for spin-2 particles, JHEP 09 (2003), 001, [hep-ph/0306182].

[54] T. Ohl, Vegas revisited: Adaptive Monte Carlo integration beyond factorization, Comput. Phys. Commun. 120 (1999), 13-19, [hep-ph/9806432].

[55] F. Cachazo, P. Svrček and E. Witten, MHV vertices and tree amplitudes in gauge theory, JHEP 09 (2004), 006, [hep-th/0403047].

[56] T. Gleisberg, S. Höche, F. Krauss and R. Matyskiewicz, How to calculate colourful cross sections efficiently, arXiv:0808.3672 [hep-ph].

[57] T. Gleisberg and S. Höche, Comix, a new matrix element generator, arXiv:0808.3674 [hep-ph].

[58] S. Dawson, Radiative corrections to Higgs boson production, Nucl. Phys. B359 (1991), 283-300.

[59] A. Bredenstein, S. Dittmaier and M. Roth, Four-fermion production at gamma gamma colliders: I. Lowest-order predictions and anomalous couplings, Eur. Phys. J. C36 (2004), 341-363, [hep-ph/0405169].

[60] A. Denner, S. Dittmaier, M. Roth and D. Wackeroth, Predictions for all processes e+ e- ¿ 4fermions + gamma, Nucl. Phys. B560 (1999), 33-65, [hep-ph/9904472]. S. Dittmaier and M. Roth, LUSIFER: A LUcid approach to SIx FERmion production, Nucl. Phys. B642 (2002), 307-343, [hep-ph/0206070].

[61] http://mlm.home.cern.ch/mlm/mcwshop03/mcwshop.html.

[62] J. Rosiek, Complete set of Feynman rules for the minimal supersymmetric extension of the standard model, Phys. Rev. D41 (1990), 3464. J. Rosiek, Complete set of Feynman rules for the MSSM ERRATUM, hep-ph/9511250.

[63] A. Denner, H. Eck, O. Hahn and J. Kublbeck, Feynman rules for fermion number violating interactions, Nucl. Phys. B387 (1992), 467-484.

[64] B. C. Allanach et al., The Snowmass points and slopes: Benchmarks for SUSY searches, hep-ph/0202233. 
[65] B. C. Allanach, SOFTSUSY: A program for calculating supersymmetric spectra, Comput. Phys. Commun. 143 (2002), 305-331, [hep-ph/0104145]. A. Djouadi, J.-L. Kneur and G. Moultaka, SuSpect: A Fortran code for the Supersymmetric and Higgs particle spectrum in the MSSM, Comput. Phys. Commun. 176 (2007), 426-455, [hep-ph/0211331]. W. Porod, SPheno, a program for calculating supersymmetric spectra, SUSY particle decays and SUSY particle production at $e^{+} e^{-}$ colliders, Comput. Phys. Commun. 153 (2003), 275-315, [hep-ph/0301101].

[66] B. Allanach et al., SUSY Les Houches Accord 2, arXiv:0801.0045 [hep-ph].

[67] D. Berdine, N. Kauer and D. Rainwater, Breakdown of the Narrow Width Approximation for New Physics, Phys. Rev. Lett. 99 (2007), 111601, [hep-ph/0703058].

[68] C. F. Uhlemann and N. Kauer, Narrow-width approximation accuracy, arXiv:0807 .4112 [hep-ph].

[69] T. Plehn, D. Rainwater and P. Skands, Squark and gluino production with jets, Phys. Lett. B645 (2007), 217-221, [hep-ph/0510144]. J. Alwall, M.-P. Le, M. Lisanti and J. G. Wacker, Searching for Gluinos at the Tevatron, arXiv:0803.0019 [hep-ph].

[70] J. Alwall, S. de Visscher and F. Maltoni, QCD radiation in the production of heavy colored particles at the $L H C$, arXiv:0810.5350 [hep-ph].

[71] G. C. Cho et al., Weak boson fusion production of supersymmetric particles at the LHC, Phys. Rev. D73 (2006), 054002, [hep-ph/0601063].

[72] T. Han, J. D. Lykken and R.-J. Zhang, On Kaluza-Klein states from large extra dimensions, Phys. Rev. D59 (1999), 105006, [hep-ph/9811350].

[73] G. F. Giudice, R. Rattazzi and J. D. Wells, Quantum gravity and extra dimensions at high-energy colliders, Nucl. Phys. B544 (1999), 3-38, [hep-ph/9811291].

[74] J. L. Hewett, Indirect collider signals for extra dimensions, Phys. Rev. Lett. 82 (1999), 4765-4768, [hep-ph/9811356].

[75] K. Hagiwara, P. Konar, Q. Li, K. Mawatari and D. Zeppenfeld, Graviton production with 2 jets at the LHC in large extra dimensions, JHEP 04 (2008), 019, [arXiv:0801.1794 [hep-ph]].

[76] K. Hagiwara, R. D. Peccei, D. Zeppenfeld and K. Hikasa, Probing the weak boson sector in $e^{+} e^{-} \rightarrow$ $W^{+} W^{-}$, Nucl. Phys. B282 (1987), 253.

[77] F. Gangemi, G. Montagna, M. Moretti, O. Nicrosini and F. Piccinini, Electroweak physics in sixfermion final states at future $e^{+} e^{-}$colliders, hep-ph/0001065.

[78] G. J. Gounaris, J. Layssac and F. M. Renard, Off-shell structure of the anomalous $Z$ and $\gamma$ selfcouplings, Phys. Rev. D62 (2000), 073012, [hep-ph/0005269].

[79] U. Baur and D. Zeppenfeld, Probing the $W W \gamma$ vertex at future hadron colliders, Nucl. Phys. B308 (1988), 127.

[80] F. J. Botella and L.-L. Chau, Anticipating the Higher Generations of Quarks from Rephasing Invariance of the Mixing Matrix, Phys. Lett. B168 (1986), 97. G. W.-S. Hou, Charmless Rare B Decays, Heavy Quarks and a Fourth Generation, Invited talk given at ' 87 DPF Meeting of the APS, Salt Lake City, Utah, Jan 14-17, 1987. W.-S. Hou, A. Soni and H. Steger, Charmless Decays of Bottom Mesons and a Fourth Generation, Phys. Rev. Lett. 59 (1987), 1521.

[81] O. Antunano, J. H. Kuhn and G. Rodrigo, Top Quarks, Axigluons and Charge Asymmetries at Hadron Colliders, Phys. Rev. D77 (2008), 014003, [arXiv:0709.1652 [hep-ph]].

[82] K. Agashe, A. Belyaev, T. Krupovnickas, G. Perez and J. Virzi, LHC signals from warped extra dimensions, Phys. Rev. D77 (2008), 015003, [hep-ph/0612015].

[83] C. Kilic, S. Schumann and M. Son, Searching for Multijet Resonances at the LHC, arXiv:0810.5542 [hep-ph]. 
[84] N. D. Christensen and C. Duhr, FeynRules - Feynman rules made easy, arXiv:0806.4194 [hep-ph].

[85] M. Bengtsson and T. Sjöstrand, A comparative study of coherent and non-coherent parton shower evolution, Nucl. Phys. B289 (1987), 810.

[86] T. Sjöstrand, A model for initial state parton showers, Phys. Lett. B157 (1985), 321.

[87] S. Catani, S. Dittmaier and Z. Trocsanyi, One-loop singular behaviour of QCD and SUSY QCD amplitudes with massive partons, Phys. Lett. B500 (2001), 149-160, [hep-ph/0011222].

[88] G. Corcella and M. H. Seymour, Matrix element corrections to parton shower simulations of heavy quark decay, Phys. Lett. B442 (1998), 417-426, [hep-ph/9809451]. K. Hamilton and P. Richardson, A simulation of QCD radiation in top quark decays, JHEP 02 (2007), 069, [hep-ph/0612236].

[89] S. Mrenna and P. Richardson, Matching matrix elements and parton showers with HERWIG and PYTHIA, JHEP 05 (2004), 040, [hep-ph/0312274]. N. Lavesson and L. Lonnblad, Merging parton showers and matrix elements - back to basics, JHEP 04 (2008), 085, [arXiv:0712.2966 [hep-ph]].

[90] V. M. Abazov et al., D $\emptyset$ collaboration, Measurement of the ratios of the $Z / \gamma^{*}+\geq n$ jet production cross sections to the total inclusive $Z / \gamma^{*}$ cross section in $p \bar{p}$ collisions at $\sqrt{s}=1.96 \mathrm{TeV}$, Phys. Lett. B658 (2008), 112-119, [hep-ex/0608052]. Y. S. Chung, CDF collaboration, $W / Z+$ jets and $Z p(T)$ measurements at Tevatron, FERMILAB-CONF-06-535-E. M. Voutilainen, DO QCD studies, hep-ex/0610002. G. Hesketh, CDF and D0 collaboration, $W$ and $Z$ (plus jets) production and asymmetries at 1.96-TeV, hep-ex/0605073. J. Hegeman, D0 collaboration, Jet production in the D0 experiment: Measurements and data-to-Monte Carlo comparisons, Nucl. Phys. Proc. Suppl. 174 (2007), 7-10. V. M. Abazov et al., D0 collaboration, Measurement of differential $Z / \gamma^{*}$ + jet $+X$ cross sections in $p \bar{p}$ collisions at $\sqrt{s}=1.96-T e V$, Phys. Lett. B669 (2008), 278-286, [arXiv:0808.1296 [hep-ex]]. C. Neu, CDF collaboration, $W^{ \pm} / Z+$ Jets and $W^{ \pm} / Z+$ Heavy Flavor Jets at the Tevatron, arXiv:0806.0825 [hep-ex]. A. Harel, CDF collaboration, Monte Carlo for top background at the Tevatron, arXiv:0807.4127 [hep-ex]. . V. M. Abazov, D0 collaboration, Evidence of $W W+W Z$ production with lepton + jets final states in proton-antiproton collisions at $\sqrt{s}=1.96 \mathrm{TeV}$, arXiv:0810.3873 [hep-ex].

[91] D $\emptyset$ collaboration, $Z+j e t$ production in the $D \emptyset$ experiment: A comparison between data and the PYTHIA and SHERPA Monte Carlos, D $\emptyset$ note 5066, http://www-d0.fnal.gov/Run2Physics/ WWW/results/prelim/HIGGS/H15/.

[92] T. Aaltonen et al., CDF collaboration, Measurement of the cross section for $W$-boson production in association with jets in p p collisions at $\sqrt{s}=1.96 \mathrm{TeV}$, Phys. Rev. D77 (2008), 011108, [arXiv: 0711.4044 [hep-ex]].

[93] A. Schälicke and F. Krauss, Implementing the ME+PS merging algorithm, JHEP 07 (2005), 018, [hep-ph/0503281].

[94] F. Krauss, A. Schalicke, S. Schumann and G. Soff, Simulating $W / Z+$ jets production at the Tevatron, Phys. Rev. D70 (2004), 114009, [hep-ph/0409106].

[95] F. Krauss, A. Schälicke, S. Schumann and G. Soff, Simulating $W / Z+$ jets production at the CERN LHC, Phys. Rev. D72 (2005), 054017, [hep-ph/0503280].

[96] T. Gleisberg, F. Krauss, A. Schälicke, S. Schumann and J.-C. Winter, Studying $W^{+} W^{-}$production at the Fermilab Tevatron with SHERPA, Phys. Rev. D72 (2005), 034028, [hep-ph/0504032].

[97] S. Catani, Y. L. Dokshitzer, M. Olsson, G. Turnock and B. R. Webber, New clustering algorithm for multijet cross sections in $e^{+} e^{-}$annihilation, Phys. Lett. B269 (1991), 432-438. S. Catani, Y. L. Dokshitzer, M. H. Seymour and B. R. Webber, Longitudinally-invariant $k_{\perp}$-clustering algorithms for hadron-hadron collisions, Nucl. Phys. B406 (1993), 187-224. S. Catani, Y. L. Dokshitzer and B. R. Webber, The $k_{\perp}$ clustering algorithm for jets in deep inelastic scattering and hadron collisions, Phys. Lett. B285 (1992), 291-299.

[98] G. C. Blazey et al., Run II jet physics, hep-ex/0005012. 
[99] V. M. Abazov et al., D0 collaboration, Measurement of the shape of the boson transverse momentum distribution in $p \bar{p} \rightarrow Z / \gamma^{*} \rightarrow e e+X$ events produced at $\sqrt{s}=1.96 \mathrm{TeV}$, Phys. Rev. Lett. 100 (2008), 102002, [arXiv:0712.0803 [hep-ex]].

[100] T. Aaltonen et al., CDF - Run II collaboration, Measurement of Inclusive Jet Cross Sections in $Z / \gamma^{*}(\rightarrow e e)+$ jets Production in pp Collisions at $\sqrt{s}=1.96$ TeV, Phys. Rev. Lett. 100 (2008), 102001, [arXiv:0711.3717 [hep-ex]].

[101] G. Rodrigo and F. Krauss, Resummed jet rates for heavy quark production in $e^{+} e^{-}$annihilation, Eur. Phys. J. C33 (2004), 457-459, [hep-ph/0309325].

[102] F. Abe et al., CDF collaboration, Measurement of Double Parton Scattering in $\bar{p} p$ collisions at $\sqrt{s}=1.8 \mathrm{TeV}$, Phys. Rev. Lett. 79 (1997), 584-589. F. Abe et al., CDF collaboration, Double parton scattering in $\bar{p} p$ collisions at $\sqrt{s}=1.8 \mathrm{TeV}$, Phys. Rev. D56 (1997), 3811-3832. D. E. Acosta et al., CDF collaboration, Underlying event in hard interactions at the Fermilab Tevatron $\bar{p} p$ collider, Phys. Rev. D70 (2004), 072002, [hep-ex/0404004].

[103] T. Affolder et al., CDF collaboration, Charged jet evolution and the underlying event in protonantiproton collisions at 1.8 TeV, Phys. Rev. D65 (2002), 092002.

[104] R. Kirschner, Generalized Lipatov-Altarelli-Parisi equations and jet calculus rules, Phys. Lett. B84 (1979), 266. A. M. Snigirev, QCD status of factorization ansatz for double parton distributions, Phys. Rev. D68 (2003), 114012, [hep-ph/0304172].

[105] K. Hamacher and M. Weierstall, The Next Round of Hadronic Generator Tuning Heavily Based on Identified Particle Data, hep-ex/9511011. P. Abreu et al., DELPHI collaboration, Tuning and test of fragmentation models based on identified particles and precision event shape data, Z. Phys. C73 (1996), 11-60.

[106] B. Andersson, G. Gustafson, G. Ingelman and T. Sjöstrand, Parton Fragmentation and String Dynamics, Phys. Rept. 97 (1983), 31-145. B. Andersson, G. Gustafson and T. Sjöstrand, A Model for Baryon Production in Quark and Gluon Jets, Nucl. Phys. B197 (1982), 45. B. Andersson, The Lund model, Camb. Monogr. Part. Phys. Nucl. Phys. Cosmol. 7 (1997), 1-471.

[107] in preparation.

[108] A. Deur, V. Burkert, J. P. Chen and W. Korsch, Determination of the effective strong coupling constant $\alpha_{s, g_{1}}\left(Q^{2}\right)$ from CLAS spin structure function data, Phys. Lett. B665 (2008), 349-351, [arXiv: 0803.4119 [hep-ph]].

[109] C. Amsler et al., Particle Data Group collaboration, Review of particle physics, Phys. Lett. B667 (2008), 1.

[110] P. Richardson, Spin correlations in Monte Carlo simulations, JHEP 11 (2001), 029, [hep-ph/0110108].

[111] M. Krämer, J. H. Kühn, M. L. Stong and P. M. Zerwas, Prospects of measuring the parity of Higgs particles, Z. Phys. C64 (1994), 21-30, [hep-ph/9404280].

[112] M. Gell-Mann and M. Levy, The Axial Vector Current in Beta Decay, Nuovo Cim. 16 (1960), 705.

[113] Kühn, Johann H. and Santamaría, A., $\tau$ Decays to pions, Z. Phys. C48 (1990), 445-452.

[114] S. Weinberg, Phenomenological Lagrangians, Physica A96 (1979), 327. J. Gasser and H. Leutwyler, Chiral Perturbation Theory to One Loop, Ann. Phys. 158 (1984), 142. J. Gasser and H. Leutwyler, Chiral perturbation theory: Expansions in the mass of the strange quark, Nucl. Phys. B250 (1985), 465. G. Ecker, J. Gasser, A. Pich and E. de Rafael, The role of resonances in chiral perturbation theory, Nucl. Phys. B321 (1989), 311.

[115] S. Anderson et al., CLEO collaboration, Hadronic structure in the decay $\tau^{-} \rightarrow \pi^{-} \pi^{0} \nu_{\tau}$, Phys. Rev. D61 (2000), 112002, [hep-ex/9910046]. 
[116] M. Neubert, Heavy-quark symmetry, Phys. Rept. 245 (1994), 259-396, [hep-ph/9306320]. I. Caprini, L. Lellouch and M. Neubert, Dispersive bounds on the shape of $\bar{B} \rightarrow D^{(*)} l \bar{\nu}$ form factors, Nucl. Phys. B530 (1998), 153-181, [hep-ph/9712417]. J. D. Richman and P. R. Burchat, Leptonic and semileptonic decays of charm and bottom hadrons, Rev. Mod. Phys. 67 (1995), 893-976, [hep-ph/9508250].

[117] N. Isgur, D. Scora, B. Grinstein and M. B. Wise, Semileptonic B and D decays in the quark model, Phys. Rev. D39 (1989), 799. D. Scora and N. Isgur, Semileptonic meson decays in the quark model: An update, Phys. Rev. D52 (1995), 2783-2812, [hep-ph/9503486]. J. L. Goity and W. Roberts, Soft pion emission in semileptonic B-meson decays, Phys. Rev. D51 (1995), 3459-3477, [hep-ph/9406236].

[118] P. Ball and R. Zwicky, New results on $B \rightarrow \pi, K, \eta$ decay form factors from light-cone sum rules, Phys. Rev. D71 (2005), 014015, [hep-ph/0406232]. P. Ball and R. Zwicky, $B_{d, s} \rightarrow \rho, \omega, K^{*}, \Phi$ decay form factors from light-cone sum rules reexamined, Phys. Rev. D71 (2005), 014029, [hep-ph/0412079]. P. Ball and G. W. Jones, $B \rightarrow \eta^{\left({ }^{\prime}\right)}$ form factors in QCD, JHEP 08 (2007), 025, [arXiv:0706.3628 [hep-ph]]. T. M. Aliev and M. Savci, Semileptonic decays of pseudoscalar mesons to scalar $f_{0}$ meson, hep-ph/0701108.

[119] D. J. Lange, The EvtGen particle decay simulation package, Nucl. Instrum. Meth. A462 (2001), $152-155$.

[120] B. Aubert et al., BABAR collaboration, Measurement of the $B^{0} \rightarrow \pi^{-} l^{+} \nu$ Form-Factor Shape and Branching Fraction, and Determination of $\left|V_{u b}\right|$ with a Loose Neutrino Reconstruction Technique, Phys. Rev. Lett. 98 (2007), 091801, [hep-ex/0612020].

[121] M. Beneke, G. Buchalla, M. Neubert and C. T. Sachrajda, QCD factorization for exclusive, nonleptonic B meson decays: General arguments and the case of heavy-light final states, Nucl. Phys. B591 (2000), 313-418, [hep-ph/0006124].

[122] S. Jadach, B. F. L. Ward and Z. Wass, The precision Monte Carlo event generator $\mathcal{K} \mathcal{K}$ for two- fermion final states in $e^{+} e^{-}$collisions, Comput. Phys. Commun. 130 (2000), 260-325, [hep-ph/9912214]. S. Jadach, W. Płaczek, M. Skrzypek, B. F. L. Ward and Z. Wa̧s, Precision predictions for (un)stable $W^{+} W^{-}$pair production at and beyond LEP2 energies, Phys. Rev. D65 (2002), 093010, [hep-ph/0007012]. S. Jadach, W. Płaczek, M. Skrzypek, B. F. L. Ward and Z. Wass, The Monte Carlo program KoralW version 1.51 and the concurrent Monte Carlo KoralWEYFSWW3 with all background graphs and first order corrections to $W$ pair production, Comput. Phys. Commun. 140 (2001), 475-512, [hep-ph/0104049]. K. Hamilton and P. Richardson, Simulation of QED radiation in particle decays using the YFS formalism, JHEP 07 (2006), 010, [hep-ph/0603034].

[123] S. Dittmaier, A general approach to photon radiation off fermions, Nucl. Phys. B565 (2000), 69-122, [hep-ph/9904440]. S. Catani, S. Dittmaier, M. H. Seymour and Z. Trocsanyi, The dipole formalism for next-to-leading order QCD calculations with massive partons, Nucl. Phys. B627 (2002), 189-265, [hep-ph/0201036].

[124] S. Schumann and F. Krauss, A parton shower algorithm based on Catani-Seymour dipole factorisation, JHEP 03 (2008), 038, [arXiv:0709.1027 [hep-ph]].

[125] J.-C. Winter and F. Krauss, Initial-state showering based on colour dipoles connected to incoming parton lines, JHEP 07 (2008), 040, [arXiv:0712.3913 [hep-ph]].

[126] T. Gleisberg and F. Krauss, Automating dipole subtraction for QCD NLO calculations, Eur. Phys. J. C53 (2008), 501-523, [arXiv:0709. 2881 [hep-ph]]. 